\title{
EDITORIAL
}

\section{Achievements of Professor Satoshi Ōmura}

The Journal of Antibiotics (2017) 70, 457-491; doi:10.1038/ja.2017.36

$\mathrm{D}$ uring his 50 years of pioneering research, Professor Satoshi Ōmura, Nobel Laureate and Emeritus Editor-in-Chief of The Journal of Antibiotics, has discovered 181 groups of new microbial metabolites, encompassing nearly 500 compounds. This editorial lists those groups, together with a representative chemical structure, producing strains and the initial publication (Figure 1 and Table 1). For much of his storied career, Professor Satoshi Ōmura has also been intimately linked with The Journal of Antibiotics and we are extremely proud that he has published $87 \%$ of his initial new compound publications in this journal. Professor Ōmura's philosophy behind his success has been relatively and refreshing simple, 'Isolate the greatest variety of microorganisms especially unique species, continually develop innovative assay systems, and perseveringly screen for new and useful bioactive compounds using those microbes and assays.' The scope, scale and impact of his discoveries prove the merits of such an approach that others would do well to follow. This editorial also shows the complete list of his publication in The Journal of Antibiotics (see list of Publications of Professor Satoshi Ōmura in The Journal of Antibiotics).

\section{ACKNOWLEDGEMENTS}

I thank Dr Tomoyasu Hirose at Kitasato Institute for Life Sciences, Kitasato University, for his assistance in preparing the list of chemical structures.

Kazuro Shiomi Managing Editor of The Journal of Antibiotics, Kitasato Institute for Life Sciences, Kitasato University, Tokyo, Japan E-mail:shiomi@lisci.kitasato-u.ac.jp 


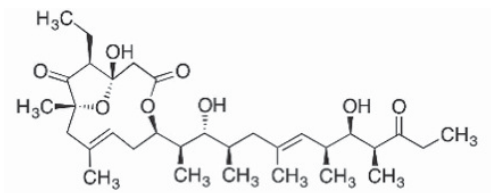

1. Actinoallolide A (2015)<smiles>OCC1OC2CC(O)C(O)C1N=CNCC2O</smiles>

4. Adechlorin (1985)

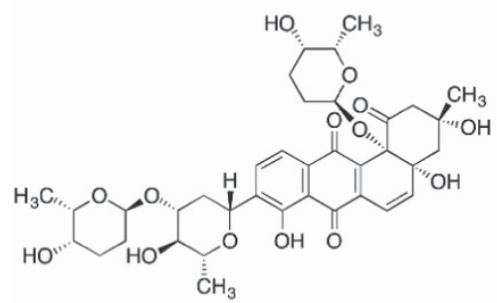

7. Aggreticin (1988)<smiles>COc1cc(C)c(C(=O)Oc2cc(C)c(C(=O)Oc3cc(C)c(C(=O)NC(C)C(=O)O)c(O)c3)c(O)c2)c(OC)c1</smiles>

10. Amidepsine A (1995)

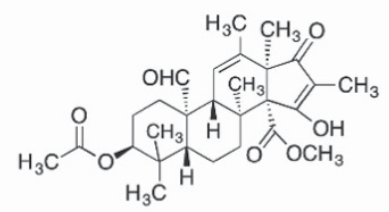

13. Andrastin A (1996)

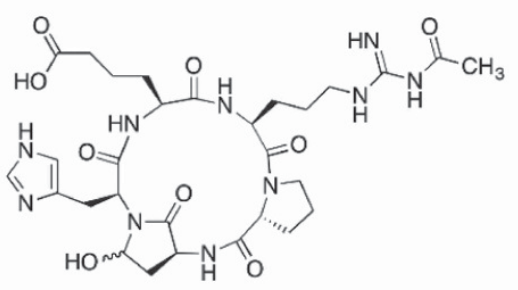

16. Argadin (2000)
1-38 ASVTIRNAOTGRLLDSNYNGNVYTLPANGGNYORWTGP 39-76 GDGTVRNAQTGRCLDSNYDGAVYTLPCNGGSYQKWLFY 77-114 SNGYIQNVETGRVLDSNYNGNVYTLPANGGNYQKWYTG

2. Actinohivin (2001)<smiles>OCC1=CC(n2cnc3c2N=CNCC3O)C(O)C1O</smiles>

5. Adecypenol (1986)<smiles>COC(=O)c1cc(OC)cc(OC)c1C(=O)c1coc(CC(C)OC(C)=O)cc1=O</smiles>

3. Actofunicone (2002)<smiles>CCC(C)CCCCCCCCCCC(=O)OCC(O)CO</smiles>

6. Aggreceride A (1986)<smiles>CCCC(=O)NC(C(=O)NC(C(=O)NC(CC(C)C)C(O)CC(=O)NC(C)C(=O)NC(Cc1ccccc1)C(O)CC(=O)O)C(C)C)C(C)C</smiles>

8. Ahpatinin A (1986)<smiles>CO[C@H](/C=C/[C@@]1(C)C=CC(=O)O1)/C(C)=C/CC[C@H](C)C(C)=O</smiles>

\section{Albocycline $\mathrm{K} 1$} (1993)<smiles>Nc1ncnc2c1ncn2CC(N)O</smiles>

11. 2'-Amino-2'-deoxyadenosine (1979)<smiles>CCCCC1C(=O)OC(C)C(NC(=O)c2cccc(NC=O)c2O)C(=O)OC1C</smiles>

14. Antimycin $A_{9}$ (2005)

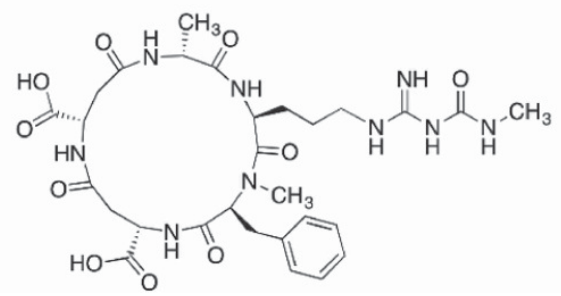

17. Argifin (2000)<smiles>NC(CCNC(CNC(Cc1c[nH]cn1)C(=O)O)C(=O)O)C(=O)O</smiles>

12. Amphistin (1997)<smiles>C=C1C(=O)C(C)(O)C(=O)OC12CCCCCC2CC</smiles>

15. Aogacillin A (2013)

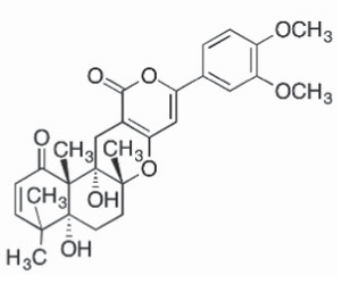

18. Arisugacin A (1995)

Figure 1 Structures of the new compounds discovered by Professor Satoshi Ōmura. 
<smiles>Cc1ccc2c(c1/C=C/C=C/C(=O)O)C(C)CC(C)C2O</smiles>

19. Arohynapene A (1994)

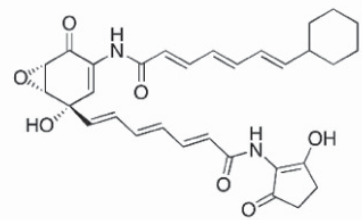

22. Asukamycin (1976)



25. Avermectin $B_{1 a}$ (1979)<smiles>CCCCC(C)[C@@H]1CC(=O)N[C@@H](Cc2ccccc2)C(=O)N[C@@H](C)C(=O)N[C@@H]([C@@H](C)CC)C(=O)O1</smiles>

28. Beauveriolide III (1999)<smiles>CCC(CC)(NC(=O)C(CCC(=O)O)NC(=O)C(Cc1c[nH]cn1)NC(=O)[C@H](CC(C)C)NC(=O)[C@H](N)Cc1ccccc1)C(=O)N[C@H]1CCCCNC1=O</smiles>

31. Calpinactam (2010)

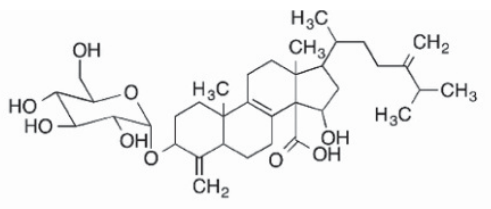

20. Ascosteroside C (2015)

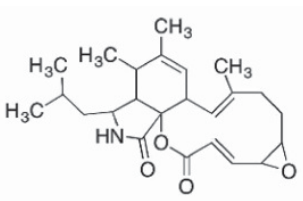

21. Aspochalasin F (1997)

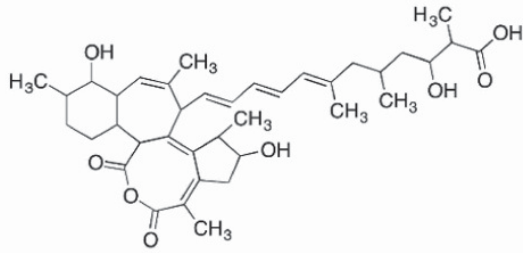

24. Aurantinin A (1976)

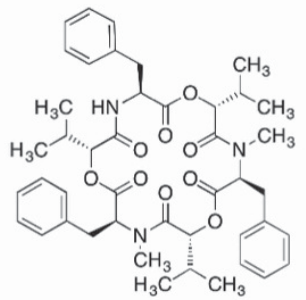

27. Beauvericin D (2004)

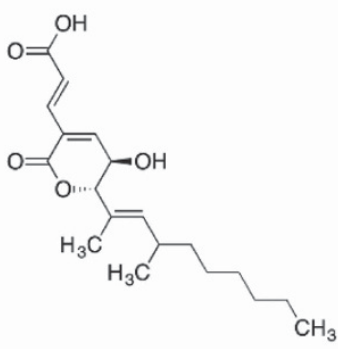

30. Biverlactone A (2011)

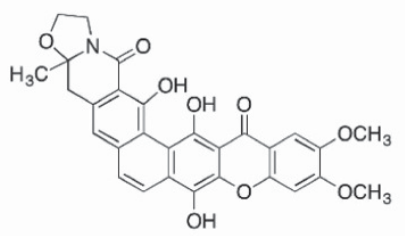

33. Cervinomycin $A_{1}$ (1982)

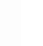

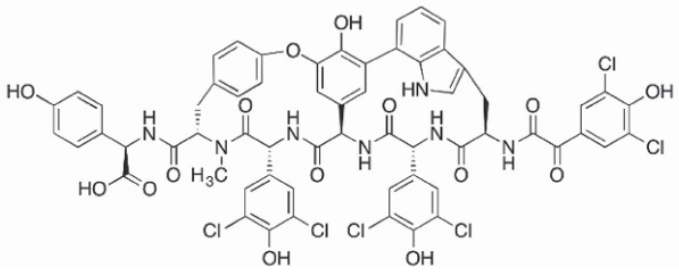

35. Chloropeptin I (1994)

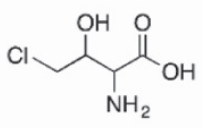

36. 4-Chlorothreonine

(1994)

Figure 1 Continued.

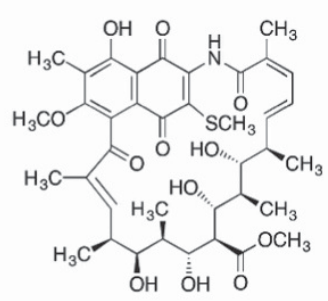

26. Awamycin (1983)<smiles>O=C1C=C(Cl)C(=O)C(CO)=C1</smiles>

(2001)
32. Cerulenin (1967)

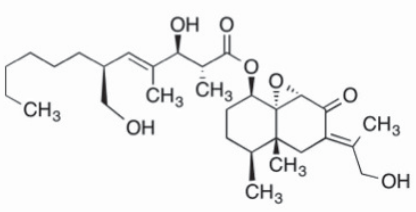

29. Berkleasmin F (2016)

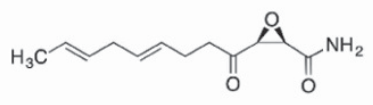




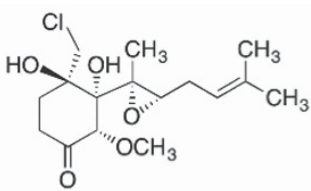

37. Chlovalicin (1996)<smiles>C=CC(C)(C)C(=O)Nc1ccccc1C(=O)CCNC(=O)CCC(NC(C)=O)C(=O)O</smiles>

40. Citrinamide A (2008)<smiles>CCCCCCCCCCCC[C@@H]1C(=O)OC(C(=O)O)[C@@]1(O)C(=O)O</smiles>

38. Cinatrin D (2015)<smiles>CC1CCCCCC(O)C(=O)/C=C/C1=O</smiles>

41. Cladospolide D (2001)<smiles>CC1=CC2(C)Oc3c(-c4ccccc4)c[nH]c(=O)c3C2C1C</smiles>

39. Citridone A (2005)

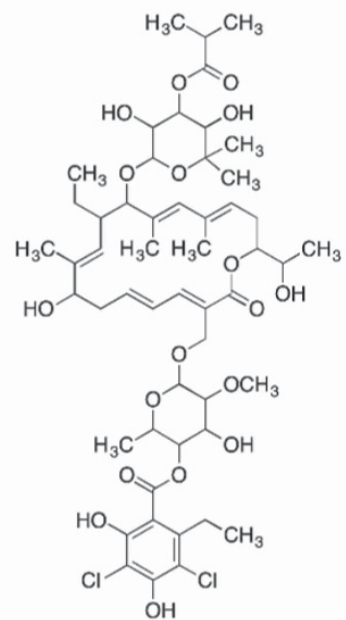

42. Clostomicin A (1986)

43. Coculnol

(2015)

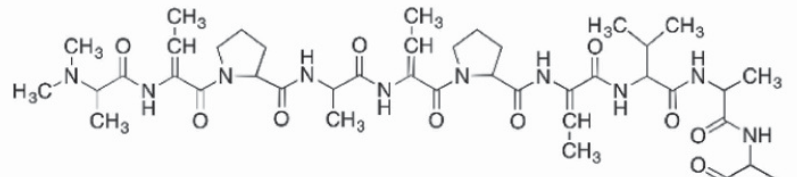<smiles>COC1=CC(=O)O/C1=C(/C)CO</smiles>

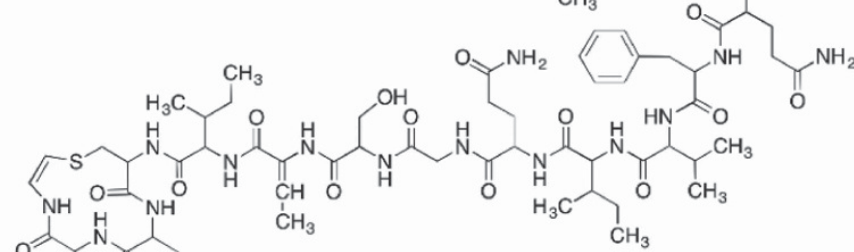

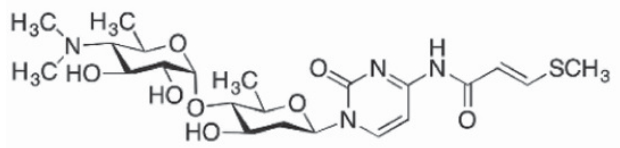

46. Cytosaminomycin A (1994)<smiles>CC(=CCO)CC1=C(CC(=O)O)[C@@]2(C)CCCC(C)(C)[C@H]2CC1</smiles>

49. Decatamariic acid (2017)

44. Cypemycin (1993)<smiles>CCCCCC1OC(=O)Cc2cc(OC)c(O)c(O)c21</smiles>

47. Cytosporone S (2013)<smiles>CC(/C=C/C1(C)C(C)C=CC(=O)C1C)=C\Cc1c(O)c(Cl)c(C)c(C=O)c1O</smiles>

50. 8',9'-Dehydroascochlorin (1994)<smiles>C=C/C(C)=C/CC1C(O)C(CSCC(NC(C)=O)C(=O)O)CC2C(C)(C)CCCC12C</smiles>

45. Cyslabdan (2008)<smiles>COc1cc(C)cc2c(=O)oc3c(C4OC(C)C(O)C(N(C)C)C4O)ccc(O)c3c12</smiles>

48. Deacetylravidomycin M (2001)<smiles></smiles>

51. Diazaquinomycin A (1982)

Figure 1 Continued. 
<smiles>COC(=O)C1CC(=O)C=C(OC)C12Oc1cc(C)cc(O)c1C2=O</smiles>

52. Dihydrobisdechlorogeodin (1996)<smiles>Oc1ccc(C[C@@H](O)[C@H](O)Cc2c[nH]c3ccccc23)cc1</smiles>

54. Diolmycin A1 (1993)<smiles>CCCCCC(=O)C(CCCC)c1oc(=O)c(CCCC)c(O)c1CCCC</smiles>

57. Elasnin (1978)

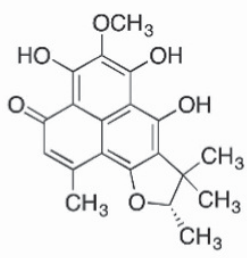

60. Erabulenol A (1998)

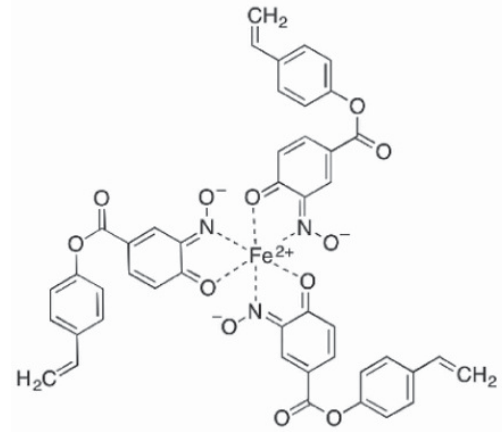

63. Ferroverdin A (1999)<smiles>CCCCCC[C@H](O)CC[C@@H](O)C[C@@H]1Cc2cc3cc(OC)c(-c4c(OC)cc5cc6c(c(O)c5c4O)C(=O)O[C@H](CC[C@H](O)CCCCC)C6)c(O)c3c(O)c2C(=O)O1</smiles>

53. Dinapinone A1 (2011)<smiles>O=C(NCCc1ccccc1)NCCc1ccccc1</smiles>

55. 1,3-Diphenethylurea (1978)

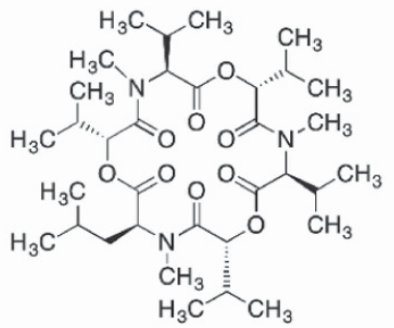

58. Enniatin D (1992)

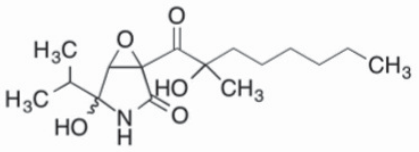

61. Euvesperin A (2016)

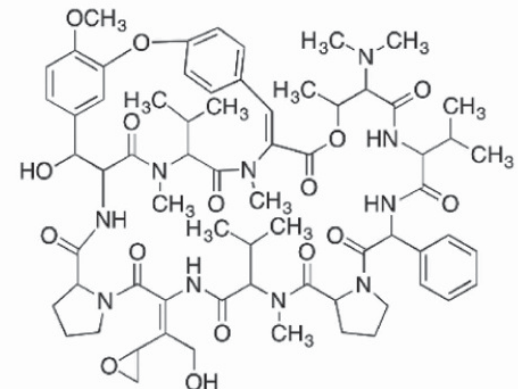

56. Dityromycin (1977)

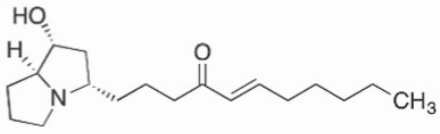

59. Epohelmin A (2004)

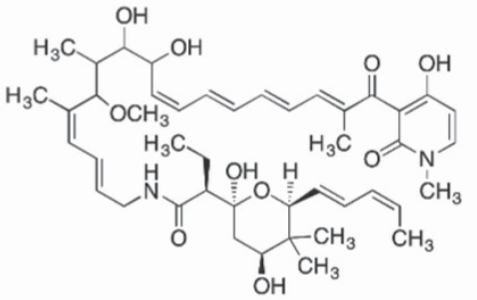

62. Factumycin (1982)<smiles>CCCC1O[C@@H]2CC(=O)O[C@H]2C2=C1C(=O)c1cccc(O)c1C2=O</smiles>

64. Frenolicin B (1978)

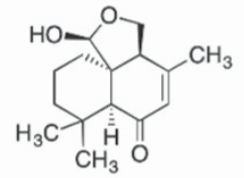

65. Fudecalone

(1995)<smiles>COC1=C(O)C2=C(O)C=C(C)c3c(O)cc(O)c(c31)C2=O</smiles>

66. Funalenone (1999)

Figure 1 Continued. 
<smiles>COC1=C(C)C(=O)c2c(cc(O)c3c2O[C@H](C)[C@@]3(C)[C@H](O)C/C=C(/C)CO)C1=O</smiles>

67. Furaquinocin A (1989)

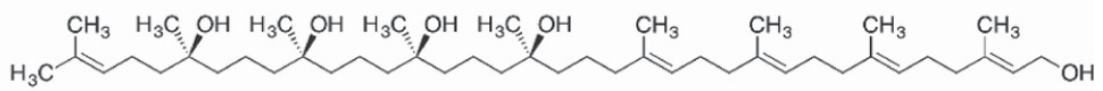

68. Glisoprenin A (1992)<smiles>C/C=C(/C)C(OC(OC1C(O)C(O)C(O)C1O)C(C)/C=C(C)/C=C/CC(C)(O)/C=C/c1nc(OC)c(OC)c(O)c1C)C(C)O</smiles>

69. Glucopiericidinol $A_{1}$ (1989)<smiles>CN1C[C@@H]2C[C@@H]3CCC(=O)[C@@H]([C@@H]4C=CCC(=O)[C@@H]34)[C@@H]2C1</smiles>

72. Herquline A (1979)<smiles>CN1CC(CNc2ccc(C(=O)NC(CCC(=O)NC(CCC(=O)O)C(=O)O)C(=O)O)cc2)=Nc2c1nc(N)[nH]c2=O</smiles>

75. 7-Hydro-8-methylpteroylglutamylglutamic acid (1987)<smiles>C/C=C/C1=CC2=CC(=O)C(C)(OC(=O)c3c(OC)cc(O)c(O)c3C)C(=O)C2=CO1</smiles>

78. 6'-Hydroxy-3'-methoxymitoruburin (2010)<smiles>CC1CC(C)C2C(C1)C(O)C(O)C(C)C2(O)/C=C/C=C/C(=O)O</smiles>

81. Hynapene A (1993)

Figure 1 Continued.

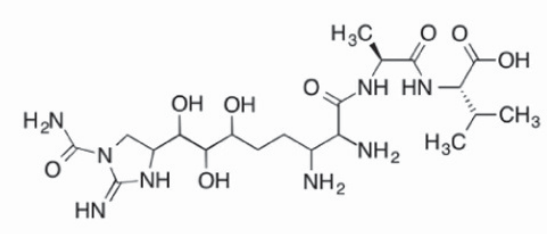

70. Guadinomine A (2008)

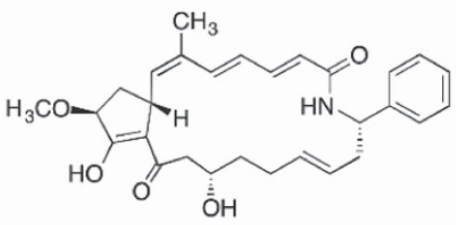

73. Hitachimycin (1982)<smiles>CC1=C(CCO)C(=O)c2ccccc2C1=O</smiles>

76. 2-(2-Hydroxyethyl)-3-methyl1,4-naphthoquinone (2000)<smiles>C=C1OC(=O)[C@@]2(C(O)C(=O)C=C[C@H]2C)[C@H]1O</smiles>

79. 6-epi-5'-Hydroxymycosporulone (1999)

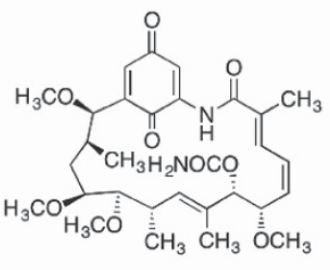

71. Herbimycin A (1979)<smiles>COc1cccc2c1C(O)c1ccc3c(c1C2O)C(=O)CC(C)(O)C3</smiles>

74. Hydranthomycin (1995)<smiles>COC(=O)/C=C/c1ncn(C)c1C/C=C(\C)CO</smiles>

77. Hydroxyfungerin A (2005)<smiles>CC(=C\C(=O)O)/C=C(\C)CC(C)CCCCC1OC(=O)C1CO</smiles>

80. Hymeglusin (1233A)

(1987)

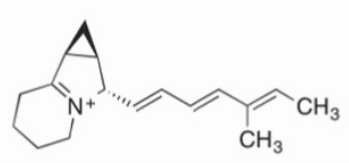

82. Iminimycin A (2016)<smiles>CCC1OC(OC(C=CC(C)C2COC(OC(N)=O)[C@@H](O)C2C)CCCC=C(C)C2OC(O)(CC(=O)OC(C(C)CC(C)C3OC3(C)CC)C(C)C(C)C)CC=C2C)CC1C</smiles>

83. Irumamycin (1982) 


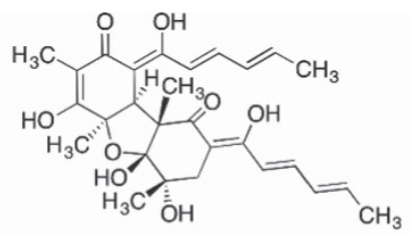

84. Isobisvertinol (2007)

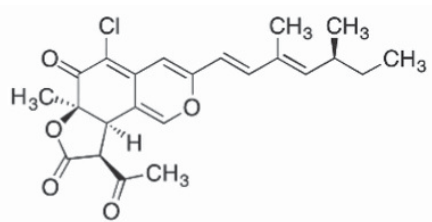

85. Isochromophilone I (1993)<smiles></smiles>

86. Jietacin A (1987)<smiles>CC[C@]1(NC(=O)N(C)C)[C@H](N)[C@@H](Nc2cccc(C(C)=O)c2)[C@@](C)(O)[C@]1(C)CO</smiles>

87. Jogyamycin (2012)

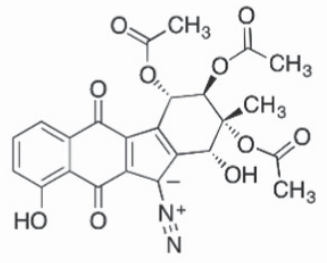

90. Kinamycin A (1971)<smiles>CC12CCC3=C(CCC4=CC(=O)[C@@H](O)[C@H](O)[C@]43C)C1CCC2O</smiles>

88. K10-0216 KA

(2014)<smiles>O=C(Cc1ccccc1)C(O)Cc1ccc(O)cc1</smiles>

91. Kurasoin A (1996)<smiles>CC(=O)/C=C/C=C/C=C/C=C/C(=O)O</smiles>

92. LA-1

(1976)<smiles>CC(=O)NC(CSC(=O)[C@H](NC(=O)C(C)C)[C@H](O)C(C)C)C(=O)O</smiles>

93. Lactacystin (1991)

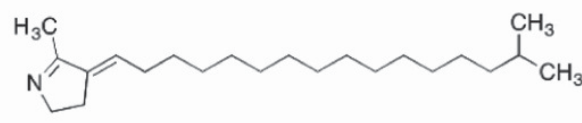

94. Lanopylin $A_{1}$ (2003)



96. Leucomycin $A_{3}$ (1966)

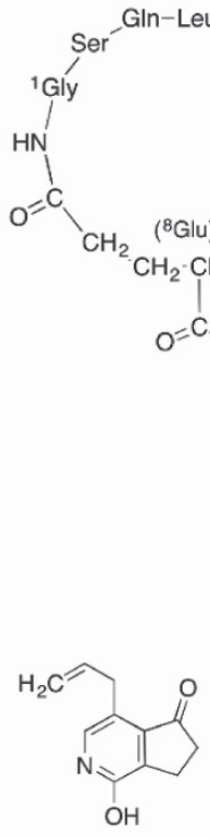

97. Louisianin A (1995)

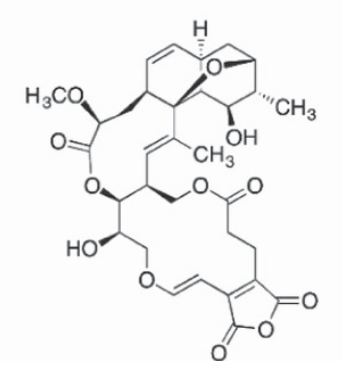

98. Luminamicin (1985)

95. Lariatin A (2007)

Figure 1 Continued. 


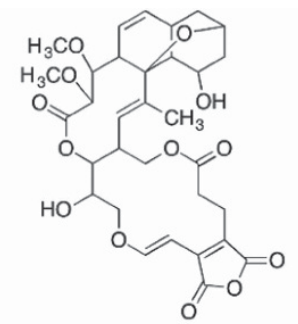

99. Lustromycin (1986)

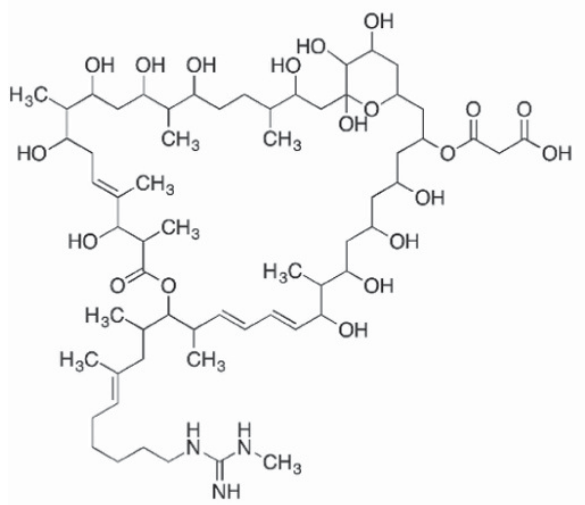

102. Malolactomycin C (1997)<smiles></smiles>

105. Monorden C (2003)

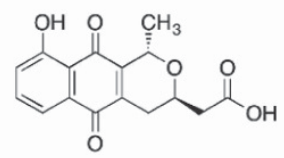

108. Nanaomycin A (1974)

111. 10-Norparvulenone (2000)

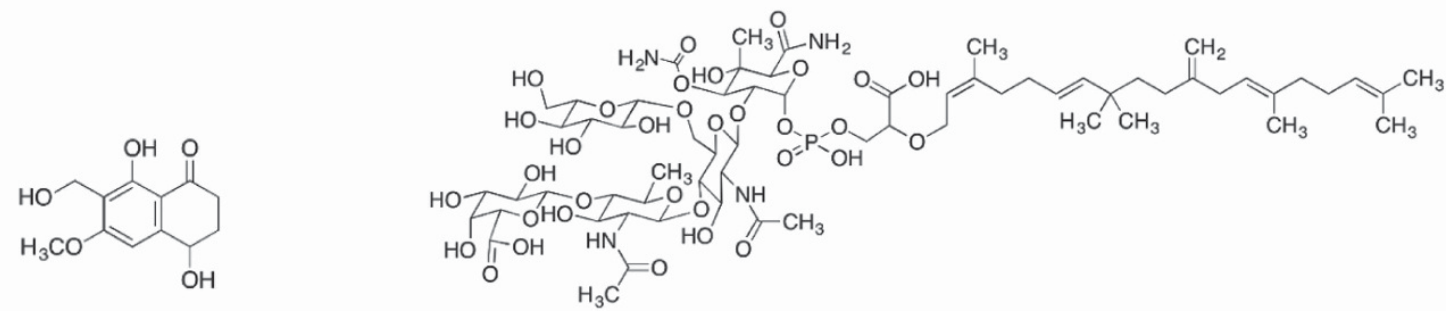

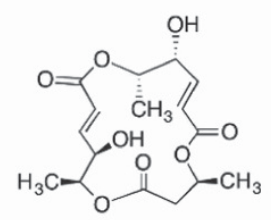

100. Macrosphelide A (1995)

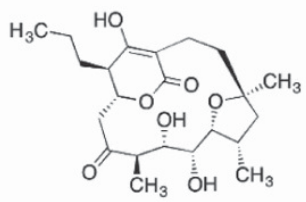

103. Mangromicin $A$

(2014)

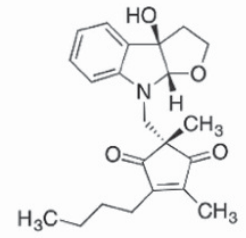

101. Madindoline A (1996)

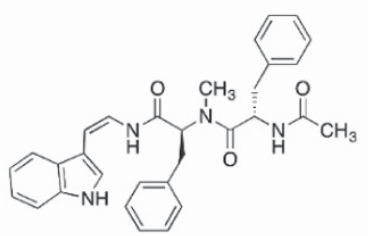

104. Miyakamide $A_{1}$ (2002)<smiles>C/C=C/C=C/C1=NCCCC1</smiles>

106. NA-337A (1974)

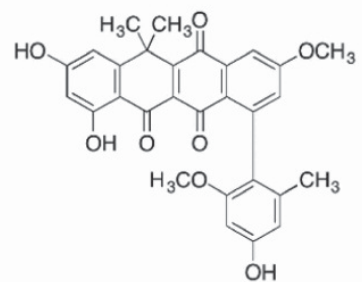

109. Naphthacemycin $A_{1}$<smiles>CCC(C)/C=C/C=C(\C)CC(C)/C=C/C=C/C1OC(=O)[C@@H](O)C[C@]1(C)O</smiles>

107. Nafuredin (2001)

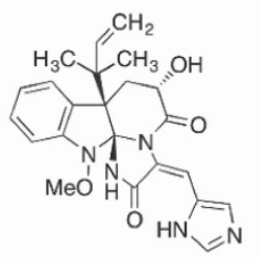

110. Neoxaline (1979)

112. Nosokomycin A (2010)

Figure 1 Continued. 


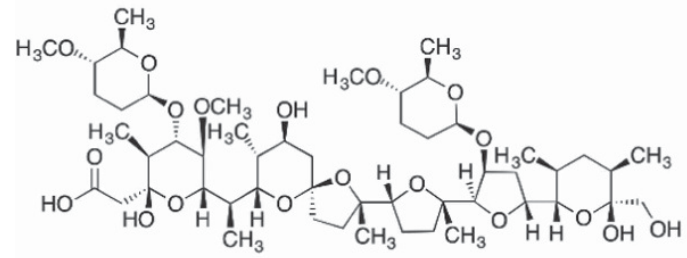

113. Octacyclomycin (1992)<smiles>COC(=O)CC1CC2=C(C(=O)c3c(O)cccc3C2=O)[C@@H](C)O1</smiles>

116. OM-173 aA (1983)<smiles>N[C@@H]1COC1C(=O)O</smiles>

117. Oxetin (1984)

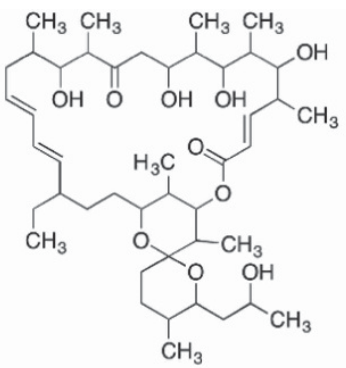

115. Oligomycin G (2001)
114. Okicenone

(1991)

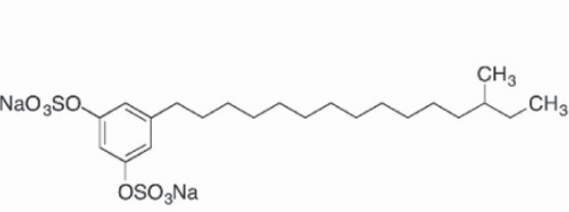

119. Panosialin D (1995)

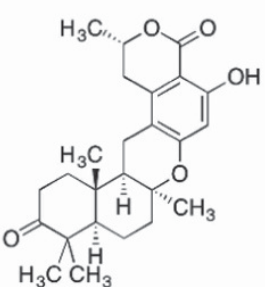

122. Pentacecilide A (2009)<smiles>Cc1cc(C)c(O)c(C(=O)CC(CC(N)=O)CC(=O)O)c1</smiles>

125. Phenatic acid A (2005)<smiles>CC(O)C1(O)C(O)C(=O)C2OC21</smiles>

123. Pentenocin A (1999)<smiles>C/C(=C\Cn1c2cccc(=O)c-2nc2ccccc21)CCC1C(=O)CCCC1(C)C</smiles>

126. Phenazinomycin (1989)<smiles>CCC(C)CC(C)CCCCCCCCCCC(O)C(C)N</smiles>

118. Paecilaminol (2006)<smiles>CC(=O)C(C)[C@H]1O[C@H](CCO)c2cc(C)ccc2[C@H]1C</smiles>

120. Panowamycin A (2012)<smiles>C=C1C2=C(C(=O)N1OC)C(O)C(C)(O)C(CCCCCCCC)O2</smiles>

121. Paraphaeosphaeride D (2016)<smiles>CCC/C=C\c1ccccc1/C=C/C(=O)N[C@@H](Cc1ccc(O)cc1)C(=O)N(C)[C@@H](Cc1ccc(OC)c(O)c1Cl)C(=O)N(C)[C@@H](Cc1ccccc1)C(=O)OC[C@H]1NC(=O)CNC1=O</smiles>

124. Pepticinnamin E (1993)<smiles>CC1=CC(/C=C/CC(C)C(=O)C(C)(O)O)C2(NC1=O)OC(=O)O/C=C/C(C)C(C)C2Cc1ccc(O)cc1</smiles>

127. Phenochalasin A (1999)

Figure 1 Continued. 
<smiles>O=C1C(O)=C(c2ccccc2)C2CCCC12</smiles>

128. Phenopyrrozin (1995)

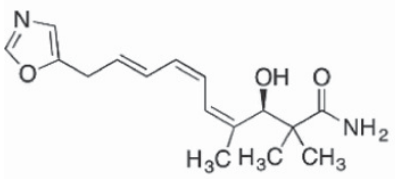

131. Phthoxazolin A (1990)<smiles>COc1cc(=O)oc(C)c1/C=C/C(C)O</smiles>

134. Pyrenocine I (2010)

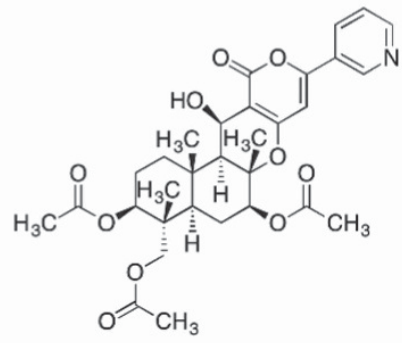

137. Pyripyropene A (1993)

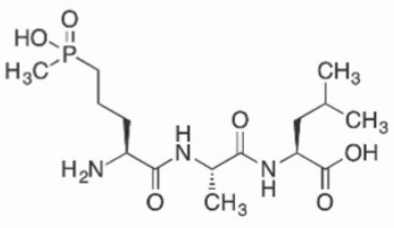

129. Phosalacine (1984)

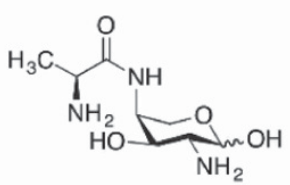

132. Prumycin (1971)

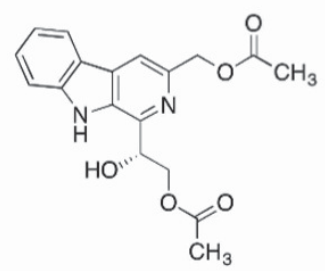

135. Pyridindolol K1 (1997)<smiles>OCc1ccc2ccccc2n1</smiles>

138. Quinoline-2-methanol (1976)<smiles>CC/C=C/C1=C2CC(C)CN=C2CC1</smiles>

136. Pyrindicin (1973)

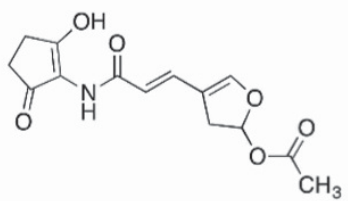

139. Reductinomycin (1981)

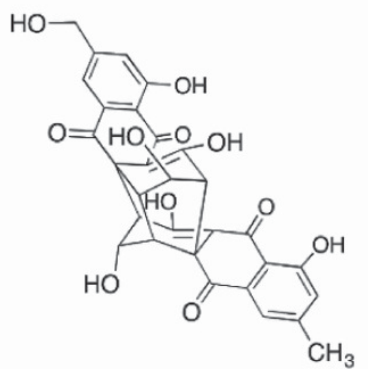

141. Rugulosin B (2010)

Figure 1 Continued.

140. Roselipin 1A (1999) 


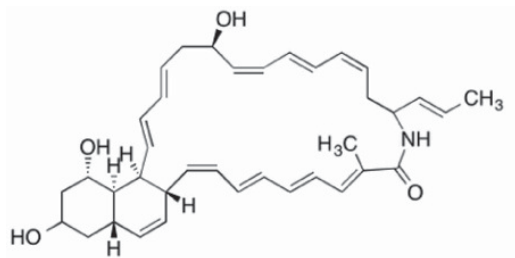

142. Sagamilactam (2016)

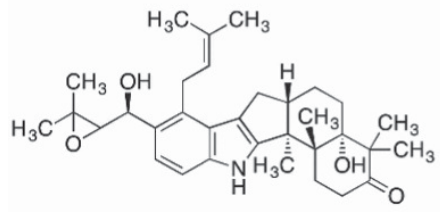

145. Sespendole (2006)

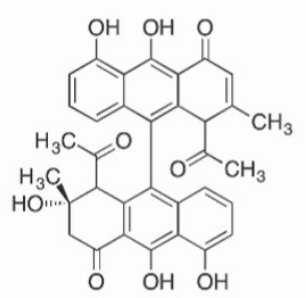

148. Setomimycin (1978)

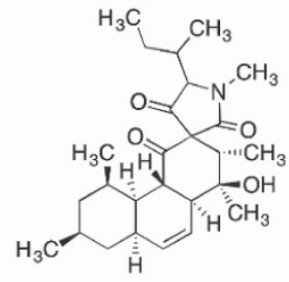

151. Spylidone (2005)

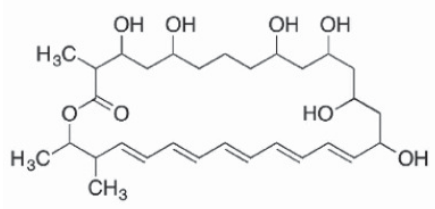

154. Takanawaene A (2003)<smiles>C=C1CCC(O)C(C)(C)C1CC/C(C)=C/CC/C(C)=C/Cc1c(O)cc(C)oc1=O</smiles>

143. Sartorypyrone D (2015)

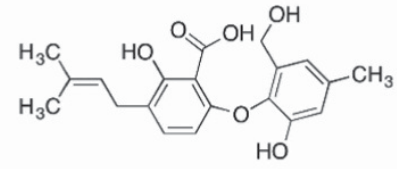

144. Secopenicillide C (2011)

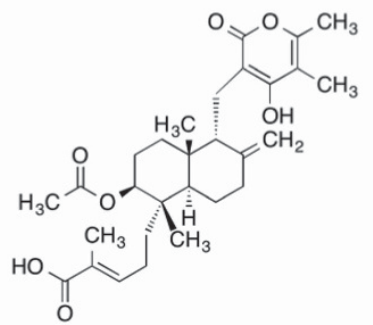

146. Sesquicillin B (2005)<smiles>CC(C)[C@H](NC(=O)C(N)CCC(N)CCC1OC2OC1C(O)C2O)C(=O)N[C@@H](C)C(=O)O</smiles>

149. Sinefungin VA (2010)

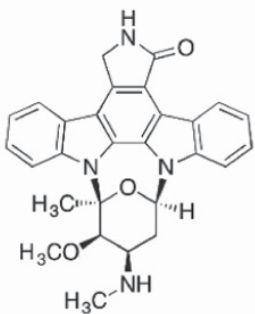

152. Staurosporine (1977)

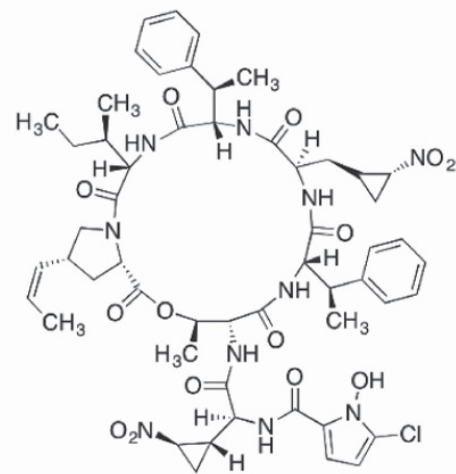

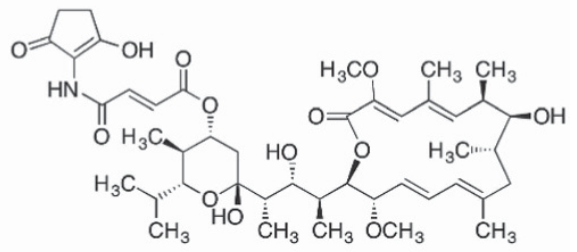

147. Setamycin (1981)

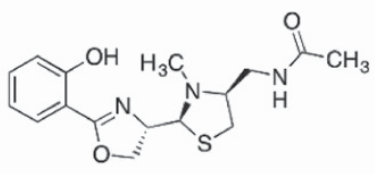

150. Spoxazomicin A (2011)

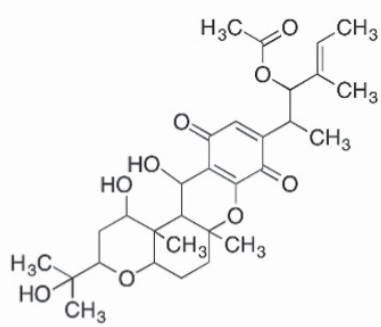

153. Stemphone B (2005)

Figure 1 Continued. 
<smiles>C=C(C(=O)O)C(CCCC(=O)OC)C(=O)OC</smiles>

156. Tensyuic acid A (2007)<smiles>CC(N)c1nc(C(=O)NCCc2c[nH]c3ccccc23)cs1</smiles>

159. TM-64 (1975)<smiles>N[C@@H](Cc1nc[nH]n1)C(=O)O</smiles>

162. L-1H-1,2,4-Triazole-3-alanine (1985)

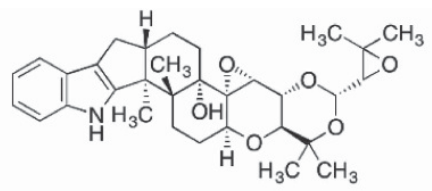

157. Terpendole A (1995)<smiles>C/C=C(/C)C(=O)OC(O)C(O)C(O)C(O)C(O)C(O)C(O)CO</smiles>

160. Trehangelin A (2013)

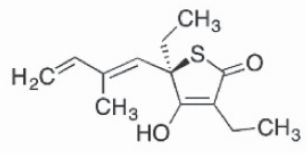

158. Thiotetromycin (1983)

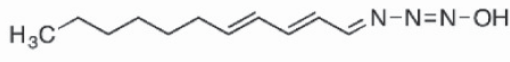

161. Triacsin A (1986)

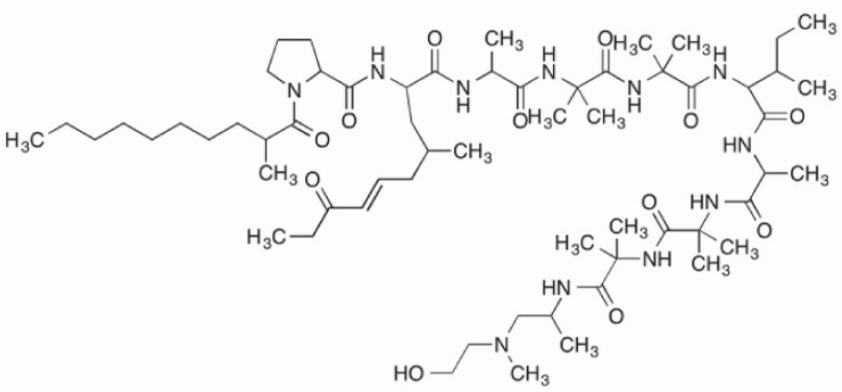

163. Trichopolyn VI (2015)

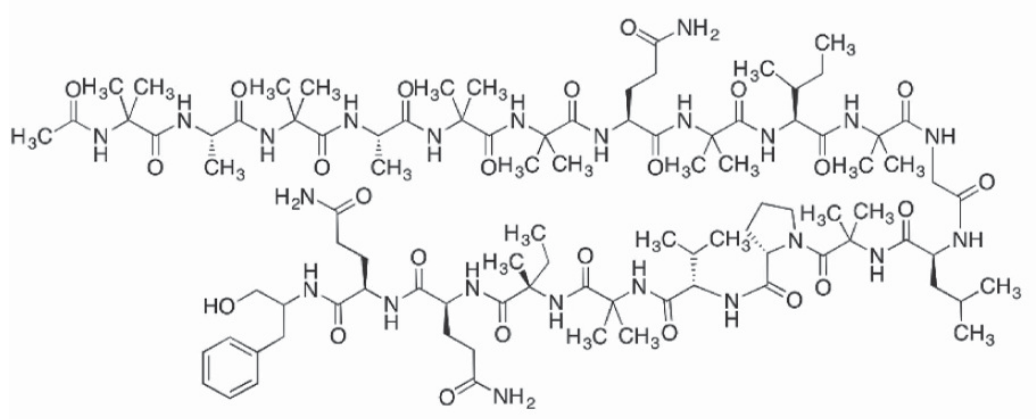

164. Trichosporin B-VIIa (2010)

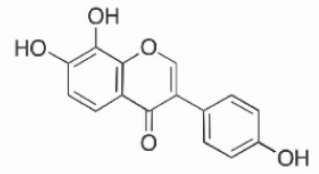

166.

4',7,8-Trihydroxyisoflavone (1989)

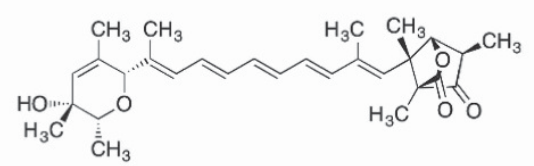

167. Ukulactone A (2011)<smiles>CCC(/C=C/C=C/C=C/CC(OC(=O)C(C)NC(=O)C1CCCCC1)C(C)C(O)/C(C)=C\CCc1cc(O)cc(NC(=O)C(C)O)c1)OC</smiles>

165. Trienomycin A (1987)

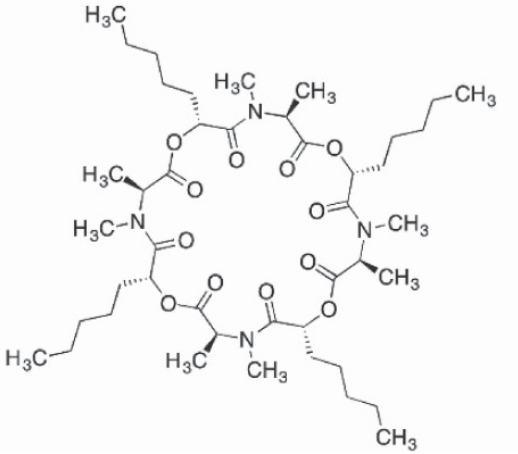

168. Verticilide (2010)

Figure 1 Continued. 
<smiles>CCCCCCCCC/C(C)=C/Cc1oc(OC)c(C)c(=O)c1C</smiles>

169. Verticipyrone (2006)<smiles>COCC1Nc2ccc(C(=O)O)cc2CC1Cl</smiles>

171. Virantmycin (1980)<smiles>CCCCC/C=C/C=C/C=C/C(=O)C1(O)C(=O)NC(CO)C1O</smiles>

172. Virgaricin A (2012)

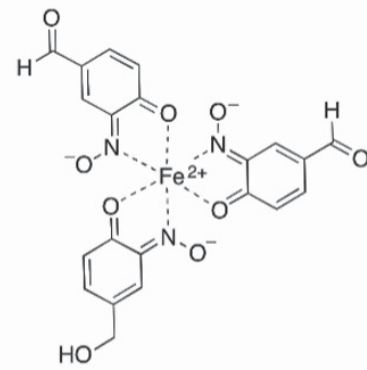

173. Viridomycin F (1999)

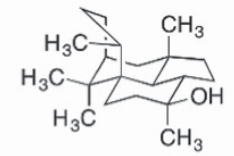

175. Viticolin A

(2011)

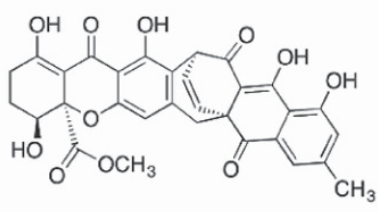

178. Xanthoquinodin $\mathrm{A} 1$ (1993)

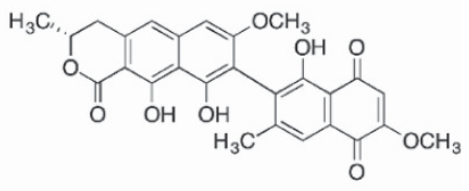

179. Xanthoradone A (2009)<smiles>COc1ccc(C2(O)c3ccccc3NC(=O)C2O)cc1</smiles>

180. Yaequinolone A1 (2006)

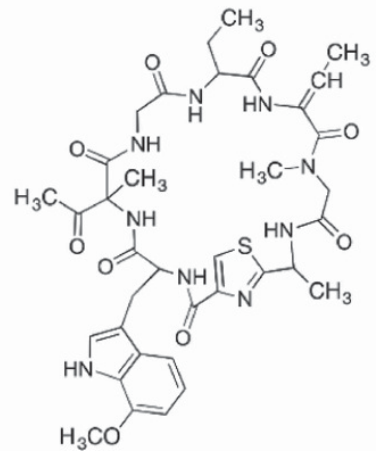

181. Zelkovamycin (1999)

Figure 1 Continued. 
Table 1 List of the producing strains and the initial publications of the new compounds

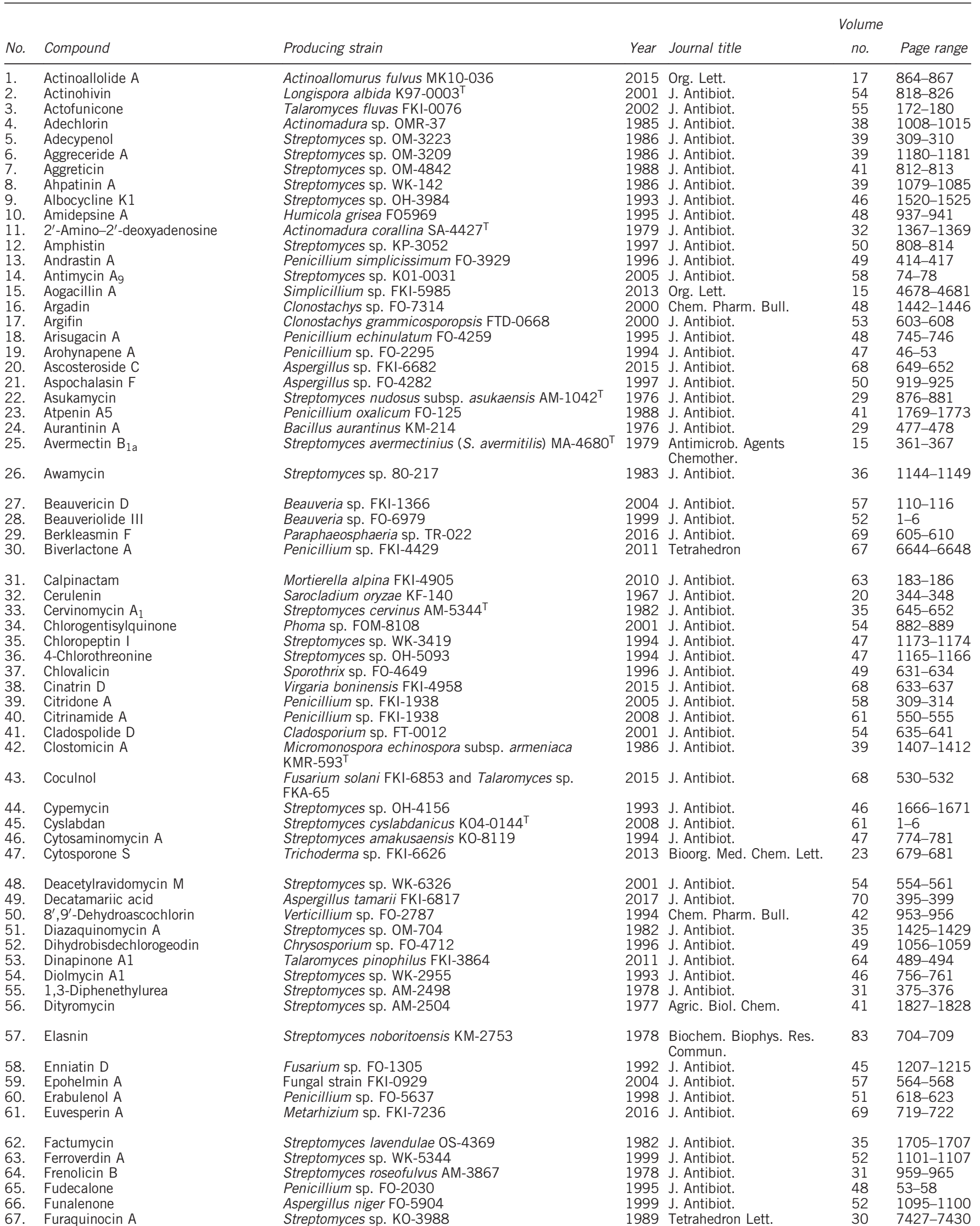


Volume

\begin{tabular}{|c|c|c|c|c|c|c|}
\hline No. & Compound & Producing strain & Year & Journal title & no. & Page range \\
\hline 68. & Glisoprenin A & Gliocladium sp. F0-1513 & 1992 & J. Antibiot. & 45 & $1202-1206$ \\
\hline 69. & Glucopiericidinol $A_{1}$ & Streptomyces sp. OM-5689 & 1989 & J. Antibiot. & 42 & $1734-1740$ \\
\hline 70. & Guadinomine A & Streptomyces sp. K01-0509 & 2008 & J. Antibiot. & 61 & $222-229$ \\
\hline 71. & Herbimycin A & Streptomyces hygroscopicus AM-3672 & 1979 & J. Antibiot. & 32 & $255-261$ \\
\hline 72. & Herquline $A$ & Penicillium herquei Fg-372 & 1979 & J. Antibiot. & 32 & $786-790$ \\
\hline 73. & Hitachimycin & Streptomyces scabrisporus KM-4927 & 1982 & Tetrahedron Lett. & 23 & $4713-4716$ \\
\hline 74. & Hydranthomycin & Streptomyces sp. K93-5305 & 1995 & J. Antibiot. & 48 & $1525-1526$ \\
\hline 75. & $\begin{array}{l}\text { 7-Hydro-8-methylpteroyl-glutamylglu- } \\
\text { tamic acid }\end{array}$ & Promicromonospora sukumoe SK-2049 & 1987 & J. Antibiot. & 40 & $251-257$ \\
\hline 76. & $\begin{array}{l}\text { 2-(2-Hydroxyethyl)-3-methyl-1,4- } \\
\text { naphthoquinone }\end{array}$ & Actinoplanes capillaceus K95-5561 & 2000 & J. Antibiot. & 53 & $1212-1214$ \\
\hline 77. & Hydroxyfungerin A & Metarhizium sp. FKI-1079 & 2005 & J. Antibiot. & 58 & $808-809$ \\
\hline 78. & $6^{\prime}$-Hydroxy-3'-methoxy-mitoruburin & Talaromyces radicus $\mathrm{FKI}-3765-2$ & 2010 & Chem. Pharm. Bull. & 58 & $829-832$ \\
\hline 79. & 6-epi-5'-Hydroxy-mycosporulone & Puruconiothyrium sporulosum FO-5050 & 1999 & J. Antibiot. & 52 & $501-504$ \\
\hline 80. & Hymeglusin $(1233 \mathrm{~A})$ & Scopulariopsis candida F-244 & 1987 & J. Antibiot. & 40 & $1356-1357$ \\
\hline 81. & Hynapene A & Penicillium sp. F0-1611 & 1993 & J. Antibiot. & 46 & 1849-1853 \\
\hline 82. & Iminimycin A & Streptomyces griseus OS-3601 & 2016 & J. Antibiot. & 69 & $611-615$ \\
\hline 83. & Irumamycin & Streptomyces subflavus subsp. irumaensis AM-3603 ${ }^{\top}$ & 1982 & J. Antibiot. & 35 & $256-257$ \\
\hline 84. & Isobisvertinol & Aspergillus clavatoranicus FKI-1746 & 2007 & Org. Lett. & 9 & $425-428$ \\
\hline 85. & Isochromophilone I & Penicillium sp. FO-2338, F0-3216 and FO-4164 & 1993 & J. Antibiot. & 46 & 1908-1911 \\
\hline 86. & Jietacin A & Streptomyces sp. KP-197 & 1987 & J. Antibiot. & 40 & $623-629$ \\
\hline 87. & Jogyamycin & Streptomyces sp. a-WM-JG-16.2 & 2012 & J. Antibiot. & 65 & $169-172$ \\
\hline 88. & K10-0216 KA & Lechevalieria aerocolonigenes K10-0216 & 2014 & J. Antibiot. & 67 & $533-539$ \\
\hline 89. & K97-0239A & Streptomyces sp. K97-0239 & 2002 & Proc. Jpn. Acad. Ser. B & 78 & $45-50$ \\
\hline 90. & Kinamycin A & Streptomyces murayamaensis KA-295 & 1971 & J. Antibiot. & 24 & $353-359$ \\
\hline 91. & Kurasoin A & Paecilomyces sp. FO-3684 & 1996 & J. Antibiot. & 49 & $932-934$ \\
\hline 92. & LA-1 & Streptomyces kitasatoensis KA-6 & 1976 & Chem. Pharm. Bull. & 24 & 3139-3143 \\
\hline 93. & Lactacystin & Streptomyces lactacystinicus OM-6519 ${ }^{\top}$ & 1991 & J. Antibiot. & 44 & $113-116$ \\
\hline 94. & Lanopylin $A_{1}$ & Streptomyces sp. K99-5041 & 2003 & J. Antibiot. & 56 & $817-826$ \\
\hline 95. & Lariatin A & Rhodococcus jostii K01-B0171 & 2007 & J. Antibiot. & 60 & $357-363$ \\
\hline 96. & Leucomycin $A_{3}$ & Streptomyces kitasatoensis $\mathrm{KA}-6^{\top}$ & 1966 & $\begin{array}{l}\text { Antimicrob. Agents } \\
\text { Chemother. }\end{array}$ & & $631-636$ \\
\hline 97. & Louisianin A & Streptomyces sp. WK-4028 & 1995 & J. Antibiot. & 48 & 1086-1089 \\
\hline 98. & Luminamicin & Streptomyces sp. OMR-59 & 1985 & J. Antibiot. & 38 & $1322-1326$ \\
\hline 99. & Lustromycin & Streptomyces sp. SK-1071 & 1986 & J. Antibiot. & 39 & $1205-1210$ \\
\hline 100. & Macrosphelide A & Paraconiothyrium sporulosum FO-5050 & 1995 & J. Antibiot. & 48 & $1435-1439$ \\
\hline 101. & Madindoline A & Streptomyces nitrosporeus K93-0711 & 1996 & J. Antibiot. & 49 & $1091-1095$ \\
\hline 102. & Malolactomycin C & Streptomyces sp. KP-3144 & 1997 & J. Antibiot. & 50 & $194-200$ \\
\hline 103. & Mangromicin A & Lechevalieria aerocolonigenes K10-0216 & 2014 & J. Antibiot. & 67 & $253-260$ \\
\hline 104. & Miyakamide $A_{1}$ & Aspergillus flavus Link var. columnaris FKI-0739 & 2002 & J. Antibiot. & 55 & $952-961$ \\
\hline 105. & Monorden C & Humicola grisea FO-2942 & 2003 & J. Antibiot. & 56 & $526-532$ \\
\hline 106. & NA-337A & Streptomyces sp. NA-337 & 1974 & Chem. Pharm. Bull. & 22 & 2916-2920 \\
\hline 107. & Nafuredin & Aspergillus niger $\mathrm{FT}-0554$ & 2001 & $\begin{array}{l}\text { Proc. Natl. Acad. Sci. } \\
\text { USA }\end{array}$ & 98 & $60-62$ \\
\hline 108. & Nanaomycin A & Streptomyces rosa subsp. notoensis OS-3996 ${ }^{\top}$ & 1974 & J. Antibiot. & 27 & $363-365$ \\
\hline 109. & Naphthacemycin $A_{1}$ & Streptomyces sp. KB-3346-5 & 2017 & J. Antibiot. & 70 & in press \\
\hline 110. & Neoxaline & Aspergillus japonicas Fg-551 & 1979 & J. Antibiot. & 32 & $781-785$ \\
\hline 111. & 10-Norparvulenone & Microsphaeropsis sp. FO-5050 & 2000 & J. Antibiot. & 53 & $1215-1218$ \\
\hline 112. & Nosokomycin A & Streptomyces sp. K04-0144 & 2010 & J. Antibiot. & 63 & $151-155$ \\
\hline 113. & Octacyclomycin & Streptomyces sp. $82-85$ & 1992 & J. Antibiot. & 45 & $1686-1691$ \\
\hline 114. & Okicenone & Streptomyces sp. KO-3599 & 1991 & J. Antibiot. & 44 & 814-818 \\
\hline 115. & Oligomycin G & Streptomyces sp. WK-6150 & 2001 & J. Antibiot. & 54 & $308-313$ \\
\hline 116. & $0 M-173 \alpha A$ & Streptomyces sp. OM-173 & 1983 & J. Antibiot. & 36 & $1268-1274$ \\
\hline 117. & Oxetin & Streptomyces sp. OM-2317 & 1984 & J. Antibiot. & 37 & 1324-1332 \\
\hline 118. & Paecilaminol & Paecilomyces sp. FKI-0550 & 2006 & J. Antibiot. & 59 & $591-596$ \\
\hline 119. & Panosialin D & Streptomyces pseudoverticillus $\mathrm{OH}-5186$ & 1995 & J. Antibiot. & 48 & $205-210$ \\
\hline 120. & Panowamycin A & Streptomyces sp. NFAT-133 & 2012 & J. Antibiot. & 65 & $197-202$ \\
\hline 121. & Paraphaeosphaeride D & Paraphaeosphaeria sp. TR-022 & 2016 & J. Antibiot. & 69 & $605-610$ \\
\hline 122. & Pentacecilide A & Talaromyces cecidicola FKI-3765-1 & 2009 & J. Antibiot. & 62 & 207-211 \\
\hline 123. & Pentenocin A & Trichoderma hamatum F0-6903 & 1999 & J. Antibiot. & 52 & $754-757$ \\
\hline 124. & Pepticinnamin E & Streptomyces sp. OH-4652 & 1993 & J. Antibiot. & 46 & $222-228$ \\
\hline 125. & Phenatic acid $A$ & Streptomyces sp. K03-0132 & 2005 & J. Antibiot. & 58 & $252-259$ \\
\hline 126. & Phenazinomycin & Streptomyces sp. WK-2057 & 1989 & J. Antibiot. & 42 & 1037-1042 \\
\hline 127. & Phenochalasin A & Phomopsis sp. FT-0211 & 1999 & J. Antibiot. & 52 & $851-856$ \\
\hline 128. & Phenopyrrozin & Penicillium sp. FO-2047 & 1995 & J. Antibiot. & 48 & $1413-1418$ \\
\hline 129. & Phosalacine & Kitasatospora phosalacinea KA-338 ${ }^{\top}$ & 1984 & J. Antibiot. & 37 & $829-835$ \\
\hline 130. & Phthoramycin & Streptomyces sp. WK-1875 & 1988 & J. Antibiot. & 41 & 1910-1912 \\
\hline 131. & Phthoxazolin A & Streptomyces sp. OM-5714 and KO-7888 & 1990 & J. Antibiot. & 43 & 1034-1036 \\
\hline
\end{tabular}


Volume

\begin{tabular}{|c|c|c|c|c|c|c|}
\hline No. & Compound & Producing strain & Year & Journal title & no. & Page range \\
\hline 132. & Prumycin & Streptomyces kagawaensis $\mathrm{F}-1028^{\top}$ & 1971 & J. Antibiot. & 24 & 900-901 \\
\hline 133. & Purpactin A & Talaromyces purpurogenus FO-608 & 1991 & J. Antibiot. & 44 & $136-143$ \\
\hline 134. & Pyrenocine I & Paecilomyces sp. FKI-3573 & 2010 & J. Antibiot. & 63 & $559-561$ \\
\hline 135. & Pyridindolol K1 & Streptomyces nitrosporeus K93-0711 & 1997 & J. Antibiot. & 50 & $189-193$ \\
\hline 136. & Pyrindicin & Streptomyces griseoflavus subsp. pyrindicus NA-15 ${ }^{\top}$ & 1973 & Chem. Pharm. Bull. & 21 & 2048-2049 \\
\hline 137. & Pyripyropene A & Aspergillus fumigatus FO-1289 & 1993 & J. Antibiot. & 46 & 1168-1169 \\
\hline 138. & Quinoline-2-methanol & Kitasatoa griseophaeus PO-1227 $7^{\top}$ & 1976 & J. Antibiot. & 29 & $797-803$ \\
\hline 139. & Reductinomycin & Streptomyces xanthochromogenus AM-6201 & 1981 & J. Antibiot. & 34 & $1222-1223$ \\
\hline 140. & Roselipin $1 \mathrm{~A}$ & Gliocladium roseum KF-1040 & 1999 & J. Antibiot. & 52 & $689-694$ \\
\hline 141. & Rugulosin B & Talaromyces radicus FKI-3765-2 & 2010 & Org. Lett. & 12 & $1572-1575$ \\
\hline 142. & Sagamilactam & Actinomadura sp. K13-0306 & 2016 & J. Antibiot. & 69 & $818-824$ \\
\hline 143. & Sartorypyrone D & Neosartorya fischeri F0-5897 & 2015 & J. Antibiot. & 68 & $403-405$ \\
\hline 144. & Secopenicillide C & $\begin{array}{l}\text { Talaromyces pinophilus FKI-5653 and Trichoderma } \\
\text { harzianum FKI-5655 }\end{array}$ & 2011 & J. Antibiot. & 64 & $769-774$ \\
\hline 145. & Sespendole & Cordana terrestris FKA-25 & 2006 & J. Antibiot. & 59 & 93-97 \\
\hline 146. & Sesquicillin B & Albophoma sp. FKI-1778 & 2005 & J. Antibiot. & 58 & $397-404$ \\
\hline 147. & Setamycin & Kitasatospora setae $\mathrm{KM}-6054^{\top}$ & 1981 & J. Antibiot. & 34 & $1253-1256$ \\
\hline 148. & Setomimycin & Streptomyces pseudovenezuelae AM-2947 & 1978 & J. Antibiot. & 31 & 1091-1098 \\
\hline 149. & Sinefungin VA & Streptomyces sp. K05-0175 & 2010 & J. Antibiot. & 63 & $673-679$ \\
\hline 150. & Spoxazomicin A & Streptosporangium oxazolinicum K07-0460 & 2011 & J. Antibiot. & 64 & 297-302 \\
\hline 151. & Spylidone & Phoma sp. FKI-1840 & 2005 & J. Antibiot. & 58 & $338-345$ \\
\hline 152. & Staurosporine & $\begin{array}{l}\text { Saccharothrix aerocolonigenes subsp. staurosporeus } \\
\text { AM- } 2282^{\top}\end{array}$ & 1977 & J. Antibiot. & 30 & $275-282$ \\
\hline 153. & Stemphone B & Aspergillus sp. FKI-2136 & 2005 & J. Antibiot. & 58 & $695-703$ \\
\hline 154. & Takanawaene A & Streptomyces sp. K99-5278 & 2003 & J. Antibiot. & 56 & $448-453$ \\
\hline 155. & Takaokamycin & Streptomyces sp. AC-1978 & 1984 & J. Antibiot. & 37 & $700-705$ \\
\hline 156. & Tensyuic acid $A$ & Aspergillus niger FKI-2342 & 2007 & Chem. Pharm. Bull. & 55 & $1338-1341$ \\
\hline 157. & Terpendole A & Albophoma yamanashiensis FO-2546 & 1995 & J. Antibiot. & 48 & $1-4$ \\
\hline 158. & Thiotetromycin & Streptomyces sp. OM-674 & 1983 & J. Antibiot. & 36 & $109-114$ \\
\hline 159. & TM-64 & Thermoactinomyces antibioticus TM-64 & 1975 & J. Antibiot. & 28 & $609-610$ \\
\hline 160. & Trehangelin A & Polymorphospora rubra K07-0510 & 2013 & J. Antibiot. & 66 & $311-317$ \\
\hline 161. & Triacsin A & Streptomyces sp. SK-1894 & 1986 & J. Antibiot. & 39 & $1211-1218$ \\
\hline 162. & L-1 H-1,2,4-Triazole-3-alanine & Streptomyces sp. KM-10329 & 1985 & J. Antibiot. & 38 & $1110-1111$ \\
\hline 163. & Trichopolyn VI & Trichoderma brevicompactum FKI-6324 & 2015 & J. Gen. Appl. Microbiol. & 61 & $82-87$ \\
\hline 164. & Trichosporin B-VIIa & Trichoderma polysporum FKI-4452 & 2010 & J. Antibiot. & 63 & $331-333$ \\
\hline 165. & Trienomycin A & Streptomyces sp. 83-16 & 1987 & J. Antibiot. & 40 & $1768-1772$ \\
\hline 166. & 4',7,8-Trihydroxyisoflavone & Streptomyces sp. OH-1049 & 1989 & J. Antibiot. & 42 & 1344-1349 \\
\hline 167. & Ukulactone A & Talaromyves allahabadensis FKI-3389 & 2011 & Tetrahedron & 67 & $6582-6586$ \\
\hline 168. & Verticilide & Acremonium variecolor $\mathrm{FKI}-1033$ & 2010 & J. Antibiot. & 63 & $77-82$ \\
\hline 169. & Verticipyrone & Metapochonia rubescens FKI-1083 & 2006 & J. Antibiot. & 59 & $785-790$ \\
\hline 170. & Vineomycin $A_{1}$ & Streptomyces matensis subsp. vineus OS $-4742^{\top}$ & 1977 & J. Antibiot. & 30 & $908-916$ \\
\hline 171. & Virantmycin & Streptomyces nitrosporeus AM-2722 & 1980 & J. Antibiot. & 33 & $1395-1396$ \\
\hline 172. & Virgaricin A & Virgaria boninensis FKI-4860 & 2012 & J. Antibiot. & 65 & $139-141$ \\
\hline 173. & Viridomycin F & Streptomyces sp. K96-0188 & 1999 & J. Antibiot. & 52 & $61-64$ \\
\hline 174. & Virustomycin A & Streptomyces sp. AM-2604 & 1982 & J. Antibiot. & 35 & 1632-1637 \\
\hline 175. & Viticolin A & Penicillium viticola $\mathrm{FKI}-4410$ & 2011 & J. Antibiot. & 64 & $183-188$ \\
\hline 176. & Wickerol A & Trichoderma atroviride FKI-3737 & 2012 & Tetrahedron & 68 & 9267-9271 \\
\hline 177. & X-14952B & Streptomyces sp. X-14952B & 1985 & J. Antibiot. & 38 & $674-676$ \\
\hline 178. & Xanthoquinodin A1 & Humicola sp. F0-888 & 1993 & J. Antibiot. & 46 & $749-755$ \\
\hline 179. & Xanthoradone A & Talaromyces radicus FKI-3765-2 & 2009 & J. Antibiot. & 62 & $431-434$ \\
\hline 180. & Yaequinolone $\mathrm{A} 1$ & Penicillium sp. FKI-2140 & 2006 & J. Antibiot. & 59 & $646-651$ \\
\hline 181. & Zelkovamycin & Streptomyces sp. K96-0670 & 1999 & J. Antibiot. & 52 & $29-33$ \\
\hline
\end{tabular}

\section{PUBLICATIONS OF PROFESSOR SATOSHI OMMURA IN THE JOURNAL OF ANTIBIOTICS}

1. Ōmura, Satoshi; Katagiri, Michiko; Hata, Toju. The structures of leucomycins $\mathrm{A}_{4}, \mathrm{~A}_{5}, \mathrm{~A}_{6}, \mathrm{~A}_{7}, \mathrm{~A}_{8}$, and $\mathrm{A}_{9}$. J. Antibiot. 20(4), 234-235 (1967).

2. Sano, Yoshimoto; Nomura, Setsuzo; Kamio, Yoshihiko; Ōmura, Satoshi; Hata, Tojo. Studies on cerulenin. III. Isolation and physic-chemical properties of cerulenin. J. Antibiot. 20(6), 344-348 (1967)

3. Ōmura, Satoshi; Katagiri, Michiko; Nakagawa, Akira; Sano, Yoshimoto; Nomura, Setsuzo; Hata, Tojo. Studies on cerulenin. V. Structure of cerulenin. J. Antibiot. 20(6), 349-354 (1967).

4. Ōmura, Satoshi; Katagiri, Michiko; Hata, Toju. The chemistry of leucomycins. IV. Structure of leucomycin $A_{1}$. J. Antibiot. 21(3), 199-203 (1968). 
5. Ōmura, Satoshi; Katagiri, Michiko; Hata, Toju. The chemistry of leucomycins. VI. Structures of leucomycin $\mathrm{A}_{4}, \mathrm{~A}_{5}, \mathrm{~A}_{6}, \mathrm{~A}_{7}, \mathrm{~A}_{8}$, and A9. J. Antibiot. 21(4), 272-278 (1968).

6. Ōmura, Satoshi; Katagiri, Michiko; Umezawa, Iwao; Komiyama, Kanki; Maekawa, Tose; Sekikawa, Kenji; Matsumae, Akihiro; Hata, Toju. Structure-biological activity relations among leucomycins and their derivatives. J. Antibiot. 21(9), 532-538 (1968).

7. Onda, Masayuki; Konda, Yaeko; Noguchi, Atsuko; Ōmura, Satoshi; Hata, Toju. Revised structure for the naphthalenecarboxylic acid from carzinophilin. J. Antibiot. 22(1), 42-44 (1969).

8. Ito, Shinya; Matsuya, Tamiko; Ōmura, Satoshi; Otani, Masaru; Nakagawa, Akira; Takeshima, Hideo; Iwai, Yuzuru; Ohtani, Mariko; Hata, Toju. A new antibiotic, kanamycin. J. Antibiot. 23 (6), 316-317 (1970).

9. Ōmura, Satoshi; Hironaka, Yukino; Hata, Toju. Chemistry of leucomycin. IX. Identification of leucomycin $\mathrm{A}_{3}$ with josamycin. J. Antibiot. 23(10), 511-513 (1970).

10. Hata, Toju; Ōmura, Satoshi; Iwai, Yuzuru; Nakagawa, Akira; Otani, Masaru; Ito, Shinya; Matsuya, Tamiko. A new antibiotic, kanamycin: fermentation, isolation, purification, and properties. J. Antibiot. 24(6), 353-359 (1971).

11. Sato, Tomoyasu; Yamaguchi, Kiyozumi; Katagiri, Michiko; Awaya, Juichi; Iwai, Yuzuru; Ōmura, Satoshi; Hata, Toju. Studies on antibiotic 0-2867, a new antibiotic. J. Antibiot. 24(11), 774-778 (1971).

12. Hata, Toju; Ōmura, Satoshi; Katagiri, Michiko; Atsumi, Kiyoo; Awaya, Juichi; Higashikawa, Shizuo; Yasui, Kazuomi; Terada, Haruko; Kuyama, Shimpei. A new antifungal antibiotic, prumycin. J. Antibiot. 24(12), 900-901 (1971).

13. Ōmura, Satoshi; Hironaka, Yukino; Nakagawa, Akira; Umezawa, Iwao; Hata, Toju. Antimycoplasma activities of macrolide antibiotics. J. Antibiot. 25(2), 105-108 (1972).

14. Nomura, Setsuzo; Horiuchi, Tadashi; Hata, Toju; Ōmura, Satoshi. Inhibition of sterol and fatty acid biosyntheses by cerulenin in cell-free systems of yeast. J. Antibiot. 25(6), 365-368 (1972).

15. Hata, Toju; Ōmura, Satoshi; Iwai, Yuzuru; Ohno, Hiroie; Takeshima, Hideo; Yamaguchi, Nobuhito. Studies on Penicillinase inhibitors produced by microorganisms. J. Antibiot. 25(8), 473-474 (1972).

16. Ōmura, Satoshi; Suzuki, Yōko; Nakagawa, Akira; Hata, Toju. Fast liquid chromatography of macrolide antibiotics. J. Antibiot. 26 (12), 794-796 (1973).

17. Arison, Byron H.; Ōmura, Satoshi. Revised structure of cerulenin. J. Antibiot. 27(1), 28-30 (1974).

18. Ōmura, Satoshi; Nakagawa, Akira; Suzuki, Kazuhiro; Hata, Toju; Jakubowski, Ann; Tishler, Max. Isolation and structure of leuconolide- $A_{8}$ 5,18-hemiacetal and 9-dehydro-18-ihydroleuconolide-A3. J. Antibiot. 27(2), 147-149 (1974).

19. Ōmura, Satoshi; Tanaka, Haruo; Koyama, Yasuaki; Ōiwa, Ruiko; Katagiri, Michiko; Awaya, Juichi; Nagai, Toshiaki; Hata, Toju. Nanaomycins A and B, new antibiotics produced by a strain of Streptomyces. J. Antibiot. 27(5), 363-365 (1974).

20. Ōmura, Satoshi; Nakagawa, Akira; Suzuki, Kazuhiro; Hata, Toju. New bicyclo lactone from leucomycin $A_{3}$ by alkali treatment. J. Antibiot. 27(5), 370-372 (1974).

21. Schwartz, Jeffrey L.; Katagiri, Michiko; Ōmura, Satoshi; Tishler, Max. The mechanism of prumycin action. J. Antibiot. 27(6), 379-385 (1974).

22. Satoh, Keiki; Komiyama, Kanki; Kitao, Chiaki; Iwai, Yuzuru; Atsumi, Kiyoo; Ōiwa, Ruiko; Katagiri, Michiko; Umezawa, Iwao;
Ōmura, Satoshi; Hata, Toju. Isolation and characterization of a new antitumor antibiotic OS-3256-B from Streptomyces candidus var azaticus. J. Antibiot. 27(8), 620-625 (1974).

23. Atsumi, Kiyoo; Ōiwa, Ruiko; Ōmura, Satoshi. Production of bacillin by Bacillus species strain no. KM-208 and its identity with tetaine (bacilysin). J. Antibiot. 28(1), 77-78 (1975).

24. Ōmura, Satoshi; Nakagawa, Akira. Chemical and biological studies on 16-membered macrolide antibiotics. J. Antibiot. 28(6), 401-433 (1975).

25. Ōmura, Satoshi; Suzuki, Y.; Kitao, C.; Takahashi, Y.; Konda, Y. Isolation of a new sulfur-containing basic substance from a Thermo actinomyces species. J. Antibiot. 28, 609-610 (1975).

26. Ōiwa, Ruiko; Katagiri, Michiko; Tanaka, Naohisa; Takahashi, Yoko; Satō, Keiki; Masuma, Rokuro; Ōmura, Satoshi. A new peptide antibiotic KM-8. J. Antibiot. 28(10), 819-820 (1975).

27. Awaya, Juichi; Kesado, Tadataka; Ōmura, Satoshi; Lukacs, Gabor. Preparation of ${ }^{13} \mathrm{C}$ - and ${ }^{3} \mathrm{H}$-labeled cerulenin and biosynthesis with ${ }^{13}$ C-NMR. J. Antibiot. 28(10), 824-827 (1975).

28. Tanaka, Haruo; Koyama, Yasuaki; Awaya, Juichi; Marumo, Hirofuto; Ōiwa, Ruiko; Katagiri, Michiko; Nagai, Toshiaki; Ōmura, Satoshi. Nanaomycins, new antibiotics produced by a strain of Streptomyces. I. Taxonomy, isolation, characterization and biological properties. J. Antibiot. 28(11), 860-867 (1975).

29. Tanaka, Haruo; Koyama, Yasuaki; Nagai, Toshiaki; Marumo, Hirofuto; Ōmura, Satoshi. Nanaomycins, new antibiotics produced by a strain of Streptomyces. II. Structure and biosynthesis. J. Antibiot. 28(11), 868-875 (1975).

30. Tanaka, Haruo; Marumo, Hirofuto; Nagai, Toshiaki; Okada, Masafumi; Taniguchi, Kazuyoshi; Ōmura, Satoshi. Nanaomycins, new antibiotics produced by a strain of Streptomyces. III. New component, nanaomycin $\mathrm{C}$, and biological activities of nanaomycin derivatives. J. Antibiot. 28(12), 925-930 (1975).

31. Schwartz, Jeffrey L.; Tishler, Max; Arison, Byron H.; Shafer, Henry M.; Ōmura, Satoshi. Identification of mycolutein and pulvomycin as aureothin and labilomycin respectively. J. Antibiot. 29(3), 236-241 (1976).

32. Ōmura, Satoshi; Takeshima, Hideo; Nakagawa, Akira; Miyazawa, Jun. The biosynthesis of picromycin using ${ }^{13} \mathrm{C}$ enriched precursors. J. Antibiot. 29(3), 316-317 (1976).

33. Ōmura, Satoshi; Nishikiori, Takaaki; Ōiwa, Ruiko; Iwai, Yuzuru; Masuma, Rokurou; Katagiri, Michiko. A new antibiotic KM-214 produced by a strain of Bacillus. J. Antibiot. 29(4), 477-478 (1976).

34. Ōmura, Satoshi; Iwai, Yuzuru; Suzuki,Yōko; Awaya, Juichi; Konda, Yaeko; Onda, Masayuki. Production of quinoline-2methanol and quinoline-2-methanol acetate by a new species of Kitasatoa, Kitasatoa griseophaeus. J. Antibiot. 29(8), 797-803 (1976).

35. Ōmura, Satoshi; Kitao, Chiaki; Tanaka, Haruo; Ōiwa, Ruiko; Takahashi, Yoko; Nakagawa, Akira; Shimada, Masayuki; Iwai, Yuzuru. A new antibiotic, asukamycin, produced by Streptomyces. J. Antibiot. 29(9), 876-881 (1976).

36. Ōmura, Satoshi; Miyazawa, Jun; Takeshima, Hideo; Kitao, Chiaki; Atsumi, Kiyoo; Aizawa, Minoru. Bioconversion of leucomycins and its regulation by butyrate in a producing strain. J. Antibiot.29 (10), 1131-1133 (1976).

37. Umezawa, Iwao; Komiyama, Kanki; Takeshima, Hideo; Awaya, Juichi; Ōmura, Satoshi. A new antitumor antibiotic, PO-357. J. Antibiot. 29(11), 1249-1251 (1976).

38. Ōmura, Satoshi; Miyazawa, Jun; Takeshima, Hideo; Kitao, Chiaki; Aizawa, Minoru. Induction of the bioconversion of leucomycins 
by glucose in a producing strain. J. Antibiot. 30(2), 192-193 (1977).

39. Ōmura, Satoshi; Iwai, Yuzuru; Hirano, Atsushi; Nakagawa, Akira; Awaya, Juichi; Tsuchiya, Hisae; Takahashi, Yoko; Masuma, Rokurou. A new alkaloid AM-2282 of Streptomyces origin. Taxonomy, fermentation, isolation and preliminary characterization. J. Antibiot. 30(4), 275-282 (1977).

40. Shimada, Masayuki; Honda, Hiroko; Awaya, Juichi; Ōmura, Satoshi. A new antibiotic, OS-1804. J. Antibiot. 30(4), 330-331 (1977).

41. Ōmura, Satoshi; Tanaka, Haruo; Ōiwa, Ruiko; Awaya, Juichi; Masuma, Rokurou; Tanaka, Katsumi. New antitumor antibiotics, OS-4742 $A_{1}, A_{2}, B_{1}$ and $B_{2}$ produced by a strain of Streptomyces. J. Antibiot. 30(11), 908-916 (1977).

42. Ōmura, Satoshi; Kitao, Chiaki; Miyazawa, Jun; Imai, Harumitsu; Takeshima, Hideo. Bioconversion and biosynthesis of 16-membered macrolide antibiotic, tylosin, using enzyme inhibitor: cerulenin. J. Antibiot. 31(3), 254-256 (1978).

43. Iwai, Yuzuru; Hirano, Atsushi; Awaya, Juichi; Matsuo, Shigetaka; Ōmura, Satoshi. 1,3-Diphenethylurea from Streptomyces sp. No. AM-2498. J. Antibiot. 31(4), 375-376 (1978).

44. Nishikiori, Takaaki; Masuma, Rokurou; Ōiwa, Ruiko; Katagiri, Michiko; Awaya, Juichi; Iwai, Yuzuru; Ōmura, Satoshi. Aurantinin, a new antibiotic of bacterial origin. J. Antibiot. 31 (6), 525-532 (1978).

45. Iwai, Yuzuru; Kōra, Akiko; Takahashi, Yōko; Hayashi, Takako; Awaya, Juichi; Masuma, Rokurou; Ōiwa, Ruiko; Ōmura, Satoshi. Production of deoxyfrenolicin and a new antibiotic, frenolicin B by Streptomyces roseofulvus strain AM-3867. J. Antibiot. 31(10), 959-965 (1978).

46. Ōmura, Satoshi; Tanaka, Haruo; Iwai, Yuzuru; Nishigaki, Kazue; Awaya, Juichi; Takahashi, Yōko; Masuma, Rokurou. A new antibiotic, Setomimycin, produced by a strain of Streptomyces. J. Antibiot. 31(11), 1091-1098 (1978).

47. Ohno, Hiroie; Saheki, Tsuneo; Awaya, Juichi; Nakagawa, Akira; Ōmura, Satoshi. Isolation and characterization of elasnin, a new human granulocyte elastase inhibitor produced by a strain of Streptomyces. J. Antibiot. 31(11), 1116-1123 (1978).

48. Ōmura, Satoshi; Iwai, Yuzuru; Takahashi, Yōko; Sadakane, Noriaki; Nakagawa, Akira; Ōiwa, Hitoshi; Hasegawa, Yasuhiro; Ikai, Takashi. Herbimycin, a new antibiotic produced by a strain of Streptomyces. J. Antibiot. 32(4), 255-261 (1979).

49. Okubo, Shuji; Nakamura, Nobuo; Ito, Kunio; Marumo, Hirofuto; Tanaka, Masao; Ōmura, Satoshi. Antitumor activity of prumycin. J. Antibiot. 32(4), 347-354 (1979).

50. Kasai, Masaji; Shirahata, Kunikatsu; Ishii, Shinzo; Mineura, Kazuyuki; Marumo, Hirofuto; Tanaka, Haruo; Ōmura, Satoshi. Structure of nanaomycin E, a new nanaomycin. J. Antibiot. 32(5), 442-425 (1979).

51. Kitao, Chiaki; Ikeda, Haruo; Hamada, Hidetaka; Ōmura, Satoshi. Bioconversion and biosynthesis of 16-membered macrolide antibiotics. XIII. Regulation of spiramycin I 3-hydroxyl acylase formation by glucose, butyrate, and cerulenin. J. Antibiot. 32(6), 593-599 (1979).

52. Hirano, Atsushi; Iwai, Yuzuru; Masuma, Rokurou; Tei, Kiyoizumi; Ōmura, Satoshi. Neoxaline, a new alkaloid produced by Aspergillus japonicus. Production, isolation and properties. J. Antibiot. 32(8), 781-785 (1979).

53. Ōmura, Satoshi; Hirano, Atsushi; Iwai, Yuzuru; Masuma, Rokurou. Herquline, a new alkaloid produced by Penicillium herquei. Fermentation, isolation and properties. J. Antibiot. 32(8), 786-790 (1979).

54. Ōmura, Satoshi; Tanaka, Haruo; Ōiwa, Ruiko; Nagai, Toshiaki; Koyama, Yasuaki; Takahashi, Yoko. Studies on bacterial cell wall inhibitors. VI. Screening method for the specific inhibitors of peptidoglycan synthesis. J. Antibiot. 32(10), 978-984 (1979).

55. Ōmura, Satoshi; Tanaka, Haruo; Tanaka, Yoshitake; Spiri-Nakagawa, Priska; Ōiwa, Ruiko; Takahashi, Yoko; Matsuyama, Kyoko; Iwai, Yuzuru. Studies on bacterial cell wall inhibitors. VII. Azureomycins A and B, new antibiotics produced by Pseudonocardia azurea species nova. Taxonomy of the producing organism, isolation, characterization and biological properties. J. Antibiot. 32(10), 985-994 (1979).

56. Spiri-Nakagawa, Priska; Tanaka, Yoshitake; Ōiwa, Ruiko; Tanaka, Haruo; Ōmura, Satoshi. Studies on bacterial cell wall inhibitors. VIII. Mode of action of a new antibiotic, azureomycin B, in Bacillus cereus T. J. Antibiot. 32(10), 995-1001 (1979).

57. Awaya, Juichi; Matsuyama, Kyoko; Iwai, Yuzuru; Ōmura, Satoshi; Ōiwa, Hitoshi; Hasegawa, Yasuhiro; Ikai, Takashi. Production of $9-\beta$-D-arabinofuranosyladenine by a new species of Streptomyces and its herbicidal activity. J. Antibiot. 32(10), 1050-1054 (1979).

58. Kitao, Chiaki; Hamada, Hidetaka; Ikeda, Haruo; Ōmura, Satoshi. Bioconversion and biosynthesis of 16-membered macrolide antibiotics. XV. Final steps in the biosynthesis of leucomycins. J. Antibiot. 32(10), 1055-1057 (1979).

59. Ōmura, Satoshi; Ikeda, Haruo; Kitao, Chiaki. The detection of a plasmid in Streptomyces ambofaciens KA-1028 and its possible involvement in spiramycin production. J. Antibiot. 32(10), 1058-1060 (1979).

60. Iwai, Yuzuru; Nakagawa, Akira; Nagai, Akiko; Matsuyama, Kyoko; Takahashi, Yoko; Yamashita, Mariko; Hirano, Atsushi; Ōmura, Satoshi. 2'-Amino-2'-deoxyadenosine produced by a strain of Actinomadura. J. Antibiot. 32(12), 1367-1369 (1979).

61. Okubo, Shuji; Nakamura, Nobuo; Morimoto, Makoto; Mineura, Kazuyuki; Marumo, Hirofuto; Ōmura, Satoshi. Studies on antitumor activity of prumycin. II. Studies on distribution and excretion of prumycin. J. Antibiot. 33(2), 221-225 (1980).

62. Okubo, Shuji; Nakamura, Nobuo; Morimoto, Makoto; Mineura, Kazuyuki; Marumo, Hirofuto; Ōmura, Satoshi. Studies on antitumor activity of prumycin. III. Mode of action of prumycin on HeLa S-3 cells. J. Antibiot. 33(2), 226-230 (1980).

63. Okubo, Shuji; Morimoto, Makoto; Mineura, Kazuyuki; Marumo, Hirofuto; Ōmura, Satoshi. Studies on antitumor activity of prumycin. IV. Effect of prumycin on mouse immune system. J. Antibiot. 33(2), 231-235 (1980).

64. Ohno, Hiroie; Yoshida, Masakazu; Takahashi, Yoko; Ōmura, Satoshi. Improvement of the productivity of elasnin, a specific elastase inhibitor, by Streptomyces noboritoensis KM-2753. J. Antibiot. 33(5), 474-479 (1980).

65. Kitao, Chiaki; Tanaka, Haruo; Minami, Shizuko; Ōmura, Satoshi. Bioconversion and biosynthesis of nanaomycins using cerulenin, a specific inhibitor of fatty acid and polyketide biosynthesis. J. Antibiot. 33(7), 711-716 (1980).

66. Ōmura, Satoshi; Iwai, Yuzuru; Masuma, Rokurou; Hayashi, Mamoru; Furusato, Takashi; Takagaki, Tatsuo. A new peptide antibiotic, alboleutin. J. Antibiot. 33(7), 758-759 (1980).

67. Furusaki, Akio; Matsumoto, Takeshi; Nakagawa, Akira; Ōmura, Satoshi. Herbimycin A. an ansamycin antibiotic; X-ray crystal structure. J. Antibiot. 33(7), 781-782 (1980). 
68. Marumo, Hirofuto; Kitaura, Kozo; Morimoto, Makoto; Tanaka, Haruo; Ōmura, Satoshi. The mode of action of nanaomycin A in gram-positive bacteria. J. Antibiot. 33(8), 885-890 (1980).

69. Ōmura, Satoshi; Kitao, Chiaki; Sadakane, Noriaki. The microbial transformation of tylosin by the spiramycin-producing strain, Streptomyces ambofaciens KA-1028. J. Antibiot. 33(8), 911-912 (1980).

70. Ōmura, Satoshi; Sadakane, Noriaki; Kitao, Chiaki; Matsubara, Hajime; Nakagawa, Akira. Production of mycarosyl protylonolide by a mycaminose idiotroph from the tylosin-producing strain Streptomyces fradiae KA-427. J. Antibiot. 33(8), 913-914 (1980).

71. Ōmura, Satoshi; Matsubara, Hajime; Nakagawa, Akira; Furusaki, Akio; Matsumoto, Takeshi. X-ray crystallography of protylonolide and absolute configuration of tylosin. J. Antibiot. 33(8), 915-917 (1980).

72. Iwai, Yuzuru; Nakagawa, Akira; Sadakane, Noriaki; Ōmura, Satoshi; Ōiwa, Hitoshi; Matsumoto, Shinichi; Takahashi, Masao; Ikai, Takashi; Ochiai, Yoshinori. Herbimycin B, a new benzoquinonoid ansamycin with anti-tobacco mosaic virus (-TMV) and herbicidal activities. J. Antibiot. 33(10), 1114-1119 (1980).

73. Ōmura, Satoshi; Nakagawa, Akira; Hashimoto, Hiroshi; Ōiwa, Ruiko; Iwai, Yuzuru; Hirano, Atsushi; Shibukawa, Nobuyuki; Kojima, Yasuhiko. Virantmycin, a potent antiviral antibiotic produced by a strain of Streptomyces. J. Antibiot. 33(11), 1395-1396 (1980).

74. Ōmura, Satoshi; Tanaka, Yoshitake; Tanaka, Haruo; Takahashi, Yoko; Iwai, Yuzuru. Stimulation of the production of macrolide antibiotics by magnesium phosphate and related insoluble materials. J. Antibiot.33 (12), 1568-1569 (1980).

75. Ōmura, Satoshi; Ikeda, Haruo; Matsubara, Hajime; Sadakane, Noriaki. Hybrid biosynthesis and absolute configuration of

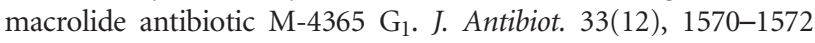
(1980).

76. Taguchi, Fumiaki; Imatani, Yoshiko; Nagaki, Daizo; Nakagawa, Akira; Ōmura, Satoshi. Selective antiviral activity of the antibiotic 2'-amino-2'-deoxyribofuranosyl adenine. J. Antibiot. 34 (3), 313-316 (1981).

77. Ōmura, Satoshi; Ikeda, Haruo; Tanaka, Haruo. Extraction and characterization of plasmids from macrolide antibiotic-producing streptomycetes. J. Antibiot. 34(4), 478-482 (1981).

78. Sakakibara, Hideo; Okekawa, Osamu; Fujiwara, Tatsuro; Otani, Masaru; Ōmura, Satoshi. Acyl derivatives of 16-membered macrolides. I. Synthesis and biological properties of 3"-O-propionylleucomycin A5 (TMS-19-Q). J. Antibiot. 34(8), 1001-1010 (1981).

79. Sakakibara, Hideo; Okekawa, Osamu; Fujiwara, Tatsuro; Aizawa, Minoru; Ōmura, Satoshi. Acyl derivatives of 16-membered macrolides. II. Antibacterial activities and serum levels of 3"-O-acyl derivatives of leucomycin. J. Antibiot. 34(8), 1011-1018 (1981).

80. Konda, Yaeko; Onda, Masayuki; Hinotozawa, Kiyoizumi; Ōmura, Satoshi. Structure of antitumor alkaloid AM-6201. J. Antibiot. 34 (9), 1222-1223 (1981).

81. Ōmura, Satoshi; Otoguro, Kazuhiko; Nishikiori, Takaaki; Ōiwa, Ruiko; Iwai, Yuzuru. Setamycin, a new antibiotic. J. Antibiot. 34 (10), 1253-1256 (1981).

82. Nakagawa, Akira; Iwai, Yuzuru; Hashimoto, Hiroshi; Miyazaki, Nobuko; Ōiwa, Ruiko; Takahashi, Yoko; Hirano, Atsushi; Shibukawa, Nobuyuki; Kojima, Yasuhiko; Ōmura, Satoshi. Virantmycin, a new antiviral antibiotic produced by a strain of Streptomyces. J. Antibiot. 34 (11), 1408-1415 (1981).
83. Imamura, Nobutaka; Kakinuma, Katsumi; Ikekawa, Nobuo; Tanaka, Haruo; Ōmura, Satoshi. The structure of vineomycin B 2 . J. Antibiot. 34(11), 1517-1518 (1981).

84. Sakakibara, Hideo; Fujiwara, Tatsuro; Aizawa, Minoru; Ōmura, Satoshi. 9-epi-Leucomycin $A_{5}$. Synthesis and antimicrobial activity. J. Antibiot. 34(12), 1577-1580 (1981).

85. Ōmura, Satoshi; Iwai, Yuzuru; Takahashi, Yoko; Kojima, Kazuko; Otoguro, Kazuhiko; Ōiwa, Ruiko. Type of diaminopimelic acid different in aerial and vegetative mycelia of setamycin-producing actinomycete KM-6054. J. Antibiot. 34(12), 1633-1634 (1981).

86. Iwai, Yuzuru; Ōmura, Satoshi. Culture conditions for screening of new antibiotics. J. Antibiot. 35(2), 123-141 (1982).

87. Ōmura, Satoshi; Tanaka, Yoshitake; Nakagawa, Akira; Iwai, Yuzuru; Inoue, Masaharu; Tanaka, Haruo. Irumamycin, a new antibiotic active against phytopathogenic fungi. J. Antibiot. 35(2), 256-257 (1982).

88. Ōmura, Satoshi; Tanaka, Haruo; Inokoshi, Junji; Sakakibara, Hideo; Fujiwara, Tatsuro. Binding of $\left[{ }^{3} \mathrm{H}\right]$ tetrahydroleucomycin $\mathrm{A}_{3}$ to Escherichia coli ribosomes and the effect of 3"-O-acyl derivatives of leucomycins on the binding. J. Antibiot. 35(4), 491-496 (1982).

89. Ikeda, Haruo; Tanaka, Haruo; Ōmura, Satoshi. Isolation and characterization of covalently closed circular DNA associated with chromosomal and membrane fraction from Streptomyces ambofaciens. J. Antibiot. 35(4), 497-506 (1982).

90. Ikeda, Haruo; Tanaka, Haruo; Ōmura, Satoshi. Genetic and biochemical features of spiramycin biosynthesis in Streptomyces ambofaciens. Curing, protoplast regeneration and plasmid transfer. J. Antibiot. 35(4), 507-516 (1982).

91. Imamura, Nobutaka; Kakinuma, Katsumi; Ikekawa, Nobuo; Tanaka, Haruo; Ōmura, Satoshi. Biosynthesis of vineomycins $A_{1}$ and $\mathrm{B}_{2}$. J. Antibiot. 35(5), 602-608 (1982).

92. Ōmura, Satoshi; Iwai, Yuzuru; Hinotozawa, Kiyoizumi; Takahashi, Yoko; Kato, Junko; Nakagawa, Akira; Hirano, Atsushi; Shimizu, Hideki; Haneda, Katsuji. Cervinomycin A1 and A2, new antibiotics active against anaerobes, produced by Streptomyces cervinus sp. nov. J. Antibiot. 35(6), 645-652 (1982).

93. Sadakane, Noriaki; Tanaka, Yoshitake; Ōmura, Satoshi. Hybrid biosynthesis of derivatives of protylonolide and M-4365 by macrolide-producing microorganisms. J. Antibiot. 35(6), 680-687 (1982).

94. Ōmura, Satoshi; Takahashi, Yōko; Iwai, Yuzuru; Tanaka, Haruo. Kitasatosporia, a new genus of the order Actinomycetales. J. Antibiot. 35(8), 1013-1019 (1982).

95. Hayashi, Maki; Unemoto, Tsutomu; Minami-Kakinuma, Shizuko; Tanaka, Haruo; Ōmura, Satoshi. The mode of action of nanaomycins D and A on a gram-negative marine bacterium Vibrio alginolyticus. J. Antibiot. 35(8), 1078-1085 (1982).

96. Masuma, Rokurou; Tanaka, Yoshitake; Ōmura, Satoshi. Enhancement of cerulenin production by a natural zeolite, an ammonium ion-trapping agent. J. Antibiot. 35(9), 1184-1193 (1982).

97. Tanaka, Haruo; Ōiwa, Ruiko; Matsukura, Shigekazu; Inokoshi, Junji; Ōmura, Satoshi. Studies on bacterial cell wall inhibitors. X. Properties of phosphor- $N$-acetylmuramoylpentapeptide transferase in peptidoglycan synthesis of Bacillus megaterium and its inhibition by amphomycin. J. Antibiot. 35(9), 1216-1221 (1982).

98. Ōmura, Satoshi; Iwai, Yuzuru; Hinotozawa, Kiyoizumi; Tanaka, Haruo; Takahashi, Yoko; Nakagawa, Akira. OM-704 A, a new antibiotic active against gram-positive bacteria produced by Streptomyces sp. J. Antibiot. 35(11), 1425-1429 (1982). 
99. Tanaka, Haruo; Minami-Kakinuma, Shizuko; Ōmura, Satoshi. Biosynthesis of nanaomycin. III. Nanaomycin A formation from nanaomycin $\mathrm{D}$ by nanaomycin $\mathrm{D}$ reductase via a hydroquinone. J. Antibiot. 35(11), 1565-1570 (1982).

100. Ōmura, Satoshi; Shimizu, Hideki; Iwai, Yuzuru; Hinotozawa, Kiyoizumi; Otoguro, Kazuhiko; Hashimoto, Hiroshi; Nakagawa, Akira. AM-2604 A, a new antiviral antibiotic produced by a strain of Streptomyces. J. Antibiot. 35 (12), 1632-1637 (1982).

101. Ōmura, Satoshi; Iwai, Yuzuru; Nakagawa, Akira; Iwata, Rimiko; Takahashi, Yoko; Shimizu, Hideki; Tanaka, Haruo. Thiotetromycin, a new antibiotic. Taxonomy, production, isolation, and physicochemical and biological properties. J. Antibiot. 36(2), 109-114 (1983).

102. Ikeda, Haruo; Inoue, Masaharu; Ōmura, Satoshi. Improvement of macrolide antibiotic-producing streptomycete strains by the regeneration of protoplasts. J. Antibiot. 36(3), 283-288 (1983).

103. Ōmura, Satoshi; Tsuzuki, Kazuo; Nakagawa, Akira; Lukacs, Gabor. Biosynthetic origin of carbons 3 and 4 of leucomycin aglycone. J. Antibiot. 36(5), 611-613 (1983).

104. Ōmura, Satoshi; Tsuzuki, Kazuo; Tanaka, Yoshitake; Sakakibara, Hideo; Aizawa, Minoru; Lukacs, Gabor. Valine as a precursor of $n$-butyrate unit in the biosynthesis of macrolide aglycone. J. Antibiot. 36(5), 614-616 (1983).

105. Sadakane, Noriaki; Tanaka, Yoshitake; Ōmura, Satoshi. Hybrid biosynthesis of a new macrolide antibiotic by a daunomycinproducing microorganism. J. Antibiot. 36(7), 921-922 (1983).

106. Ōmura, Satoshi; Sadakane, Noriaki; Tanaka, Yoshitake; Matsubara, Hajime. Chimeramycins: new macrolide antibiotics produced by hybrid biosynthesis. J. Antibiot. 36(7), 927-930 (1983).

107. Sadakane, Noriaki; Tanaka, Yoshitake; Ōmura, Satoshi. New 20-membered lactones, irumanolides I and II, produced by a mutant of Streptomyces. J. Antibiot. 36(7), 931-933 (1983).

108. Ōmura, Satoshi; Nakagawa, Akira; Aoyama, Hiroshi; Iwai, Yuzuru; Kuwahara, Masao; Furusato, Takashi. Karabemycin, a new antimetabolite of glutamine produced by a strain of streptomycete. J. Antibiot. 36(9), 1129-1135 (1983).

109. Iwai, Yuzuru; Kimura, Keiko; Takahashi, Yōko; Hinotozawa, Kiyoizumi; Shimizu, Hideki; Tanaka, Haruo; Ōmura, Satoshi. OM-173, new nanaomycin-type antibiotics produced by a strain of Streptomyces. Taxonomy, production, isolation and biological properties. J. Antibiot. 36(10), 1268-1274 (1983).

110. Sano, Hiroshi; Inoue, Michi; Yamashita, Kinya; Okachi, Ryo; Ōmura, Satoshi. Chemical modification of spiramycins. I. Synthesis of the acetal derivatives of neospiramycin I. J. Antibiot. 36(10), 1336-1344 (1983).

111. Tsuzuki, Kazuo; Ōmura, Satoshi. Syntheses and biological activities of thiotetromycin analogs. J. Antibiot. 36(11), 1589-1591 (1983).

112. Ōmura, Satoshi; Inokoshi, Junji; Matsubara, Hajime; Tanaka, Haruo. Ribosome-binding activities and antimicrobial activities of tylosin and its related compounds. J. Antibiot. 36 (12), 1709-1712 (1983).

113. Matsubara, Hajime; Inokoshi, Junji; Nakagawa, Akira; Tanaka, Haruo; Ōmura, Satoshi. Chemical modification of tylosin: synthesis of amino derivatives at C-20 position of tylosin and demycarosyltylosin. J. Antibiot. 36(12), 1713-1721 (1983).

114. Ōmura, Satoshi; Otoguro, Kazuhiko; Tanaka, Haruo. The mode of action of a novel 18-membered macrolide, virustomycin A (AM-2604 A), on Trichomonas foetus. J. Antibiot. 36(12), 1755-1761 (1983).
115. Ōmura, Satoshi; Nakagawa, Akira; Iwata, Rimiko; Hatano, Akiko. Structure of a new antibacterial antibiotic, thiotetromycin. J. Antibiot. 36(12), 1781-1782 (1983).

116. Ōmura, Satoshi; Imamura, Nobutaka; Hinotozawa, Kiyoizumi; Otoguro, Kazuhiko; Lukacs, Gabor; Faghih, Ramine; Tolmann, Richard; Arison, Byron H.; Smith, Jack L. The structure of virustomycin A. J. Antibiot. 36(12), 1783-1786 (1983).

117. Ōmura, Satoshi; Tanaka, Yoshitake; Mamada, Hiroshi; Masuma, Rokurō. Ammonium ion suppresses the biosynthesis of tylosin aglycone by interference with valine catabolism in Streptomyces fradiae. J. Antibiot. 36(12), 1792-1794 (1983).

118. Ōmura, Satoshi; Tanaka, Yoshitake; Mamada, Hiroshi; Masuma, Rokuro. Effect of ammonium ion, inorganic phosphate and amino acids on the biosynthesis of protylonolide, a precursor of tylosin aglycon. J. Antibiot. 37(5), 494-502 (1984).

119. Ōmura, Satoshi; Mamada, Hiroshi; Wang, Nanjin; Imamura, Nobutaka; Ōiwa, Ruiko; Iwai, Yuzuru; Muto, Naoki. Takaokamycin, a new peptide antibiotic produced by Streptomyces sp. J. Antibiot. 37(7), 700-705 (1984).

120. Sano, Hiroshi; Inoue, Michi; Ōmura, Satoshi. Chemical modification of spiramycins. II. Synthesis and antimicrobial activity of 4'-deoxy derivatives of neospiramycin I and their 12-(Z)-isomers. J. Antibiot. 37(7), 738-749 (1984).

121. Sano, Hiroshi; Sunazuka, Toshiaki; Tanaka, Haruo; Yamashita, Kinya; Okachi, Ryo; Ōmura, Satoshi. Chemical modification of spiramycins. III. Synthesis and antibacterial activities of 4 "-sulfonates and 4"-alkylethers of spiramycin I. J. Antibiot. 37(7), 750-759 (1984).

122. Sano, Hiroshi; Sunazuka, Toshiaki; Tanaka, Haruo; Yamashita, Kinya; Okachi, Ryo; Ōmura, Satoshi. Chemical modification of spiramycins. IV. Synthesis and in vitro and in vivo activities of 3",4"-diacylates and 3,3",4"-triacylates of spiramycin I. J. Antibiot. 37(7), 760-772 (1984).

123. Ōmura, Satoshi; Murata, Masatsune; Hanaki, Hideaki; Hinotozawa, Kiyoizumi; Ōiwa, Ruiko; Tanaka, Haruo. Phosalacine, a new herbicidal antibiotic containing phosphinothricin. Fermentation, isolation, biological activity and mechanism of action. J. Antibiot. 37(8), 829-835 (1984).

124. Ōmura, Satoshi; Hinotozawa, Kiyoizumi; Imamura, Nobutaka; Murata, Masatsune. The structure of phosalacine, a new herbicidal antibiotic containing phosphinothricin. J. Antibiot. 37(8), 939-940 (1984).

125. Ōmura, Satoshi; Matsubara, Hajime; Tsuzuki, Kazuo; Nakagawa, Akira. Chemical modification of tylosin. Thioether derivatives of tylosin and demycarosyltylosin. J. Antibiot. 37(9), 1007-1015 (1984).

126. Ikeda, Haruo; Inoue, Masaharu; Tanaka, Haruo; Ōmura, Satoshi. Interspecific protoplast fusion among macrolide-producing streptomycetes. J. Antibiot. 37(10), 1224-1230 (1984).

127. Ōmura, Satoshi; Miyano, Katsuji; Nakagawa, Akira; Sano, Hiroshi; Komiyama, Kanki; Umezawa, Iwao; Shibata, Kiyoshi; Satsumabayashi, Sadayoshi. Chemical modification and antitumor activity of herbimycin A.8,9-epoxide, 7,9-cyclic carbamate and 17 or 19-amino derivatives. J. Antibiot. 37(10), 1264-1267 (1984).

128. Ōmura, Satoshi; Murata, Masatsune; Imamura, Nobutaka; Iwai, Yuzuru; Tanaka, Haruo; Furusaki, Akio; Matsumoto, Takeshi. Oxetin, a new antimetabolite from an actinomycete. Fermentation, isolation, structure and biological activity. J. Antibiot. 37 (11), 1324-1332 (1984). 
129. Ōmura, Satoshi; Taki, Arihiro; Matsuda, Kazuko; Tanaka, Yoshitake. Ammonium ions suppress the amino acid metabolism involved in the biosynthesis of protylonolide in a mutant of Streptomyces fradiae. J. Antibiot. 37(11), 1362-1369 (1984).

130. Tanaka, Yoshitake; Masuma, Rokuro; Ōmura, Satoshi. Control of ammonium ion level for efficient nanaomycin production. J. Antibiot. 37(11), 1370-1375 (1984).

131. Ōmura, Satoshi; Tanaka, Yoshitake; Takahashi, Yoko; Chia, Iri; Inoue, Masaharu; Iwai, Yuzuru. Irumamycin, an antifungal 20-membered macrolide produced by a Streptomyces. Taxonomy, fermentation and biological properties. J. Antibiot. 37(12), 1572-1578 (1984).

132. Sano, Hiroshi; Tanaka, Haruo; Yamashita, Kinya; Okachi, Ryo; Ōmura, Satoshi. Chemical modification of spiramycins. V. Synthesis and antibacterial activity of 3'- or 4"'-de- $N$-methylspiramycin I and their $\mathrm{N}$-substituted derivatives. J. Antibiot. 38(2), 186-196 (1985).

133. Ōmura, Satoshi; Nakagawa, Akira; Imamura, Nobutaka; Kushida, Katsuhiko; Liu, Chao Min; Sello, Lilian H.; Westley, John W. Structure of a new macrolide antibiotic, X-14952B. J. Antibiot. 38(5), 674-676 (1985).

134. Ōmura, Satoshi; Imamura, Nobutaka; Kuga, Hiroshi; Ishikawa, Hideyo; Yamazaki, Yukiko; Okano, Keiko; Kimura, Keiko; Takahashi, Yoko; Tanaka, Haruo. Adechlorin, a new adenosine deaminase inhibitor containing chlorine. Production, isolation and properties. J. Antibiot. 38(8), 1008-1015 (1985).

135. Ōmura, Satoshi; Murata, Masatsune; Kimura, Keiko; Matsukura, Shigekazu; Nishihara, Tatsuro; Tanaka, Haruo. Screening for new antifolates of microbial origin and a new antifolate AM-8402. J. Antibiot. 38(8), 1016-1024 (1985).

136. Murata, Masatsune; Miyasaka, Toyoko; Tanaka, Haruo; Ōmura, Satoshi. Diazaquinomycin A, a new antifolate antibiotic, inhibits thymidylate synthase. J. Antibiot. 38(8), 1025-1033 (1985).

137. Imamura, Nobutaka; Murata, Masatsune; Yao, Tianjue; Ōiwa, Ruiko; Tanaka, Haruo; Ōmura, Satoshi. Occurrence of 1,2,4-triazole ring in actinomycetes. J. Antibiot. 38(8), 1110-1111 (1985).

138. Nakagawa, Akira; Tomoda, Hiroshi; Hao, Mai Van; Okano, Keiko; Iwai, Yuzuru; Ōmura, Satoshi. Antiviral activities of pentalenolactones. J. Antibiot. 38(8), 1114-1115 (1985).

139. Nakagawa, Akira; Tanaka, Yoshitake; Otoguro, Kazuhiko; Ōmura, Satoshi; Takiguchi, Toshimitsu; Arai, Yuko. A new antifungal antibiotic, 3'-O-decarbamoylirumamycin. J. Antibiot. 38(9), 1266-1269 (1985).

140. Ōmura, Satoshi; Iwata, Rimiko; Iwai, Yuzuru; Taga, Seiko; Tanaka, Yoshitake; Tomoda, Hiroshi. Luminamicin, a new antibiotic. Production, isolation and physico-chemical and biological properties. J. Antibiot. 38(10), 1322-1326 (1985).

141. Sano, Hiroshi; Sunazuka, Toshiaki; Tanaka, Haruo; Yamashita, Kinya; Okachi, Ryo; Ōmura, Satoshi. Chemical modification of spiramycins. VI. Synthesis and antibacterial activities of 3,3"-di-O-acyl-4"-O-sulfonyl and 3,3"-di-O-acyl-4"-O-alkyl derivatives of spiramycin I. J. Antibiot. 38(10), 1350-1358 (1985).

142. Ōmura, Satoshi; Tsuzuki, Kazuo; Iwai, Yuzuru; Kishi, Masanori; Watanabe, Shiro; Shimizu, Hideki. Anticoccidial activity of frenolicin B and its derivatives. J. Antibiot. 38(10), 1447-1448 (1985).

143. Ōmura, Satoshi; Tsuzuki, Kazuo; Sunazuka, Toshiaki; Toyota, Hajime; Takahashi, Itsuo; Itoh, Zen. Gastrointestinal motor-stimulating activity of macrolide antibiotics and the structure-activity relationship. J. Antibiot. 38(11), 1631-1632 (1985).

144. Ōmura, Satoshi; Tanaka, Haruo; Kuga, Hiroshi; Imamura, Nobutaka. Adecypenol, a unique adenosine deaminase inhibitor containing homopurine and cyclopentene rings. J. Antibiot. 39 (2), 309-310 (1986).

145. Shibata, Kiyoshi; Satsumabayashi, Sadayoshi; Sano, Hiroshi; Komiyama, Kanki; Nakagawa, Akira; Ōmura, Satoshi. Chemical modification of herbimycin A. Synthesis and in vivo antitumor activities of halogenated and other related derivatives of herbimycin A. J. Antibiot. 39(3), 415-423 (1986).

146. Tanaka, Yoshitake; Taki, Arihiro; Masuma, Rokuro; Ōmura, Satoshi. Mechanism of nitrogen regulation of protylonolide biosynthesis in Streptomyces fradiae. J. Antibiot. 39(6), 813-821 (1986).

147. Ōmura, Satoshi; Imamura, Nobutaka; Kawakita, Kazuhito; Mori, Yoko; Yamazaki, Yukiko; Masuma, Rokurou; Takahashi, Yōko; Tanaka, Haruo; Huang, Lee Yuan; Woodruff, H. Boyd. Ahpatinins, new acid protease inhibitors containing 4-amino-3-hydroxy-5-phenylpentanoic acid. J. Antibiot. 39(8), 1079-1085 (1986).

148. Ōmura, Satoshi; Nakagawa, Akira; Fukamachi, Nobuko; Otoguro, Kazuhiko; Kobayashi, Bonro. Aggreceride, a new platelet aggregation inhibitor from Streptomyces. J. Antibiot. 39 (8), 1180-1181 (1986).

149. Tomoda, Hiroshi; Iwata, Rimiko; Takahashi, Yōko; Iwai, Yuzuru; Ōiwa, Ruiko; Ōmura, Satoshi. Lustromycin, a new antibiotic produced by Streptomyces sp. J. Antibiot. 39(9), 1205-1210 (1986).

150. Ōmura, Satoshi; Tomoda, Hiroshi; Xu, Qinmin; Takahashi, Yoko; Iwai, Yuzuru. Triacsins, new inhibitors of acyl-CoA synthetase produced by Streptomyces sp. J. Antibiot. 39(9), 1211-1218 (1986).

151. Ōmura, Satoshi; Ishikawa, Hideyo; Kuga, Hiroshi; Imamura, Nobutaka; Taga, Seiko; Takahashi, Yōko; Tanaka, Haruo. Adecypenol, a unique adenosine deaminase inhibitor containing homopurine and cyclopentene rings. Taxonomy, production and enzyme inhibition. J. Antibiot. 39(9), 1219-1224 (1986).

152. Tsuzuki, Kazuo; Iwai, Yuzuru; Ōmura, Satoshi; Shimizu, Hideki; Kitajima, Nakao. Nanaomycins production by a frenolicin B-producing strain. J. Antibiot. 39(9), 1343-1345 (1986).

153. Ōmura, Satoshi; Imamura, Nobutaka; Ōiwa, Ruiko; Kuga, Hiroshi; Iwata, Rimiko; Masuma, Rokuro; Iwai, Yuzuru. Clostomicins, new antibiotics produced by Micromonospora echinospora subsp. armeniaca subsp. nov. I. Production, isolation, and physicochemical and biological properties. J. Antibiot. 39 (10), 1407-1412 (1986).

154. Takahashi, Yoko; Iwai, Yuzuru; Ōmura, Satoshi. Clostomicins, new antibiotics produced by Micromonospora echinospora subsp. armeniaca subsp. nov. II. Taxonomic study of the producing microorganism. J. Antibiot. 39(10), 1413-1418 (1986).

155. Masuma, Rokuro; Tanaka, Yoshitake; Tanaka, Hajime; Ōmura, Satoshi. Production of nanaomycin and other antibiotics by phosphate-depressed fermentation using phosphate-trapping agents. J. Antibiot. 39(11), 1557-1564 (1986).

156. Shibata, Kiyoshi; Satsumabayashi, Sadayoshi; Nakagawa, Akira; Ōmura, Satoshi. The structure and cytocidal activity of herbimycin C. J. Antibiot. 39(11), 1630-1633 (1986).

157. Nakagawa, Akira; Iwai, Yuzuru; Shimizu, Hideki; Ōmura, Satoshi. Enhanced antimicrobial activity of acetyl derivatives of cervinomycin. J. Antibiot. 39(11), 1636-1638 (1986). 
158. Spiri-Nakagawa, Priska; Fukushi, Yukiko; Maebashi, Kazunori; Imamura, Nobutaka; Takahashi, Yoko; Tanaka, Yoshitake; Tanaka, Haruo; Ōmura, Satoshi. Izupeptins A and B, new glycopeptide antibiotics produced by an actinomycete. J. Antibiot. 39(12), 1719-1723 (1986).

159. Tsuzuki, Kazuo; Matsubara, Hajime; Nakagawa, Akira; Ōmura, Satoshi. Syntheses and antimicrobial activities of 9-O-acyl derivatives of tylosin and demycarosyltylosin. J. Antibiot. 39(12) 1784-1787 (1986).

160. Tanaka, Yoshitake; Hirata, Kazuko; Takahashi, Yoko; Iwai, Yuzuru; Ōmura, Satoshi. Globopeptin, a new antifungal peptide antibiotic. J. Antibiot. 40(2), 242-244 (1987).

161. Murata, Masatsune; Tanaka, Haruo; Ōmura, Satoshi. 7-Hydro-8methylpteroylglutamylglutamic acid, a new antifolate from an actinomycete. Fermentation, isolation, structure, and biological activity. J. Antibiot. 40(3), 251-257 (1987).

162. Nakagawa, Akira; Ōmura, Satoshi; Kushida, Katsuhiko; Shimizu, Hideki; Lukacs, Gabor. Structure of cervinomycin, a novel xanthone antibiotic active against anaerobes and Mycoplasma. J. Antibiot. 40(3), 301-308 (1987).

163. Ōmura, Satoshi; Otoguro, Kazuhiko; Imamura, Nobutaka; Kuga, Hiroshi; Takahashi, Yoko; Masuma, Rokuro; Tanaka, Yoshitake; Tanaka, Haruo; Su, Xuehui; You, Entai. Jietacins A and B, new nematocidal antibiotics from a Streptomyces sp. Taxonomy, isolation, and physicochemical and biological properties. J. Antibiot. 40(5), 623-629 (1987).

164. Nakagawa, Akira; Fukamachi, Nobuko; Yamaki, Koji; Hayashi, Masahiko; Ohishi, Sachiko; Kobayashi, Bonro; Ōmura, Satoshi. Inhibition of platelet aggregation by medermycin and its related isochromanequinone antibiotics. J. Antibiot. 40(7), 1075-1076 (1987).

165. Ōmura, Satoshi; Tomoda, Hiroshi; Kumagai, Hidetoshi. Potent inhibitory effect of antibiotic 1233A on cholesterol biosynthesis which specifically blocks 3-hydroxy-3-methylglutaryl coenzyme a synthase. J. Antibiot. 40(9), 1356-1357 (1987).

166. Tomoda, Hiroshi; Igarashi, Kazuaki; Tanaka, Yoshitake; Ōmura, Satoshi. Biosynthetic preparation of labeled cerulenin with high specific radioactivity. J. Antibiot. 40(10), 1457-1460 (1987).

167. Ōmura, Satoshi; Nakagawa, Akira; Fujimoto, Tomoko; Saito, Kazunori; Otoguro, Kazuhiko; Walsh, James C. Hygromycin A, an antitreponemal substance. I. Screening method and therapeutic effect for Treponema hyodysenteriae-caused infection in CF-1 mice. J. Antibiot. 40(11), 1619-1626 (1987).

168. Nakagawa, Akira; Fujimoto, Tomoko; Ōmura, Satoshi; Walsh, James C.; Stotish, Ronald L.; George, Beverly. Hygromycin A, an antitreponemal substance. II. Therapeutic effect for swine dysentery. J. Antibiot. 40(11), 1627-1635 (1987).

169. Komiyama, Kanki; Hirokawa, Yumiko; Yamaguchi, Hiroko; Funayama, Shinji; Masuda, Kazuaki; Anraku, Yumi; Umezawa, Iwao; Ōmura, Satoshi. Antitumor activity of trienomycin A on murine tumors. J. Antibiot. 40(12), 1768-1772 (1987).

170. Masuma, Rokuro; Okuyama, Kazuko; Tanaka, Yoshitake; Hirose, Rimiko; Tomoda, Hiroshi; Iwai, Yuzuru; Ōmura, Satoshi. A new agar medium suitable for screening of anti-clostridium agents. J. Antibiot. 40(12), 1773-1775 (1987).

171. Tomoda, Hiroshi; Kumagai, Hidetoshi; Takahashi, Yoko; Tanaka, Yoshitake; Iwai, Yuzuru; Ōmura, Satoshi. F-244 (1233A), a specific inhibitor of 3-hydroxy-3-methylglutaryl coenzyme A synthase: taxonomy of producing strain, fermentation, isolation and biological properties. J. Antibiot. 41(2), 247-249 (1988).
172. Otoguro, Kazuhiko; Nakagawa, Akira; Ōmura, Satoshi. Setamycin, a 16-membered macrolide antibiotic: identification and nematocidal activity. J. Antibiot. 41(2), 250-252 (1988).

173. Tanaka, Yoshitake; Kimura, Keiko; Komagata, Yasuko; Tsuzuki, Kazuo; Tomoda, Hiroshi; Ōmura, Satoshi. Radioimmunoassay for erythromycin derivatives. J. Antibiot. 41(2), 258-260 (1988).

174. Konda, Yaeko; Nakagawa, Akira; Harigaya, Yoshihiro; Onda, Masayuki; Masuma, Rokuro; Ōmura, Satoshi. Aurantinin B, a new antimicrobial antibiotic from bacterial origin. J. Antibiot. 41 (2), 268-270 (1988).

175. Otoguro, Kazuhiko; Ōiwa, Ruiko; Iwai, Yuzuru; Tanaka, Haruo; Ōmura, Satoshi. Screening for new antitrichomonal substances of microbial origin and antitrichomonal activity of trichostatin A. J. Antibiot. 41(4), 461-468 (1988).

176. Otoguro, Kazuhiko; Liu, Zhong Xun; Fukuda, Keiko; Li, Yin; Iwai, Yuzuru; Tanaka, Haruo; Ōmura, Satoshi. Screening for new nematocidal substances of microbial origin by a new method using the pine wood nematode. J. Antibiot. 41(4), 573-575 (1988).

177. Shibata, Kiyoshi; Satsumabayashi, Sadayoshi; Sano, Hiroshi; Komiyama, Kanki; Nakagawa, Akira; Ōmura, Satoshi. Chemical modification of hitachimycin. Synthesis, antibacterial, cytocidal and in vivo antitumor activities of hitachimycin derivatives. J. Antibiot. 41(5), 614-623 (1988).

178. Ōmura, Satoshi; Nakagawa, Akira; Fukamachi, Nobuko; Miura, Satomi; Takahashi, Yoko; Komiyama, Kanki; Kobayashi, Bonro. OM-4842, a new platelet aggregation inhibitor from Streptomyces. J. Antibiot. 41(6), 812-813 (1988).

179. Uehara, Yoshimasa; Murakami, Yuko; Suzukake-Tsuchiya, Kayoko; Moriya, Yukari; Sano, Hiroshi; Shibata, Kiyoshi; Ōmura, Satoshi. Effect of herbimycin derivatives on src oncogene function in relation to antitumor activity. J. Antibiot. 41(6), 831-834 (1988).

180. Funayama, Shinji; Anraku, Yumi; Mita, Akira; Yang, Zhi Bo; Shibata, Kiyoshi; Komiyama, Kanki; Umezawa, Iwao; Ōmura, Satoshi. Structure-activity relationship of a novel antitumor ansamycin antibiotic trienomycin A and related compounds. J. Antibiot. 41(9), 1223-1230 (1988).

181. Ōmura, Satoshi; Tomoda, Hiroshi; Kimura, Keiko; Zhen, D. Z.; Kumagai, Hidetoshi; Igarashi, Kazuaki; Imamura, Nobutaka; Takahashi, Yoko; Tanaka, Yoshitake; Iwai, Yuzuru. Atpenins, new antifungal antibiotics produced by Penicillium sp. Production, isolation, physico-chemical and biological properties. J. Antibiot. 41(12), 1769-1773 (1988).

182. Ōmura, Satoshi; Tanaka, Yoshitake; Hisatome, Kazuyoshi; Miura, Satomi; Takahashi, Yoko; Nakagawa, Akira; Imai, Harumitsu. Phthoramycin, a new antibiotic active against a plant pathogen, Phytophthora sp. J. Antibiot. 41(12), 1910-1912 (1988).

183. Imamura, Nobutaka; Kuga, Hiroshi; Otoguro, Kazuhiko; Tanaka, Haruo; Ōmura, Satoshi. Structures of jietacines: unique $\alpha, \beta$-unsaturated azoxy antibiotics. J. Antibiot. 42(1), 156-158 (1989).

184. Tanaka, Haruo; Kawakita, Kazuhito; Suzuki, Hidekazu; Spiri-Nakagawa, Priska; Ōmura, Satoshi. The mode of action of cervinomycin in Staphylococcus aureus. J. Antibiot. 42(3), 431-439 (1989).

185. Nomoto, Hisayo; Katsumata, Shigeo; Takahashi, Keiichi; Funayama, Shinji; Komiyama, Kanki; Umezawa, Iwao; Ōmura, Satoshi. Structural studies on minor components of trienomycin 
group antibiotics trienomycins D and E. J. Antibiot. 42(3), 479-481 (1989).

186. Shibata, Kiyoshi; Satsumabayashi, Sadayoshi; Sano, Hiroshi; Komiyama, Kanki; Yang, Zhibo; Nakagawa, Akira; Ōmura, Satoshi. Chemical modification of hitachimycin. II. Synthesis and antitumor activities of carbonate derivatives. J. Antibiot. 42 (5), 718-726 (1989).

187. Tsuzuki, Kazuo; Yokozuka, Tadao; Murata, Masatsune; Tanaka, Haruo; Ōmura, Satoshi. Synthesis and biological activity of analogs of diazaquinomycin A, a new thymidylate synthase inhibitor. J. Antibiot. 42(5), 727-737 (1989).

188. Ōmura, Satoshi; Eda, Shigeru; Funayama, Shinji; Komiyama, Kanki; Takahashi, Yoko; Woodruff, H. Boyd. Studies on a novel antitumor antibiotic, phenazinomycin. taxonomy, fermentation, isolation, and physico-chemical and biological characteristics. J. Antibiot. 42(7), 1037-1042 (1989).

189. Shibata, Kiyoshi; Satsumabayashi, Sadayoshi; Sano, Hiroshi; Komiyama, Kanki; Yang, Zhi Bo; Nakagawa, Akira; Ōmura, Satoshi. Chemical modification of hitachimycin. III. Synthesis and antitumor activities of amino acyl derivatives. J. Antibiot. 42 (7), 1114-1123 (1989).

190. Debono, Manuel; Willard, Kevin E.; Kirst, Herbert A.; Wind, Julie A.; Crouse, Gary D.; Tao, Eddie V.; Vicenzi, Jeffrey T.; Counter, Fred T.; Ott, John L.; Ose, Earle E.; Ōmura, Satoshi. Synthesis and antimicrobial evaluation of 20-deoxo-20-(3,5dimethylpiperidin-l-yl)desmycosin (tilmicosin, EL-870) and related cyclic amino derivatives. J. Antibiot. 42(8), 1253-1267 (1989).

191. Imai, Harumitsu; Nakagawa, Akira; Ōmura, Satoshi. Biosynthesis of the antibiotic okilactomycin. J. Antibiot. 42(8), 1321-1323 (1989).

192. Nakagawa, Akira; Miura, Satomi; Imai, Harumitsu; Imamura, Nobutaka; Ömura, Satoshi. Structure and biosynthesis of a new antifungal antibiotic, phthoramycin. J. Antibiot. 42(8), 1324-1327 (1989).

193. Komiyama, Kanki; Funayama, Shinji; Anraku, Yumi; Mita, Akira; Takahashi, Yoko; Ōmura, Satoshi; Shimasaki, Hiroyuki. Isolation of isoflavonoids possessing antioxidant activity from the fermentation broth of Streptomyces sp. J. Antibiot. 42(9), 1344-1349 (1989).

194. Funayama, Shinji; Anraku, Yumi; Mita, Akira; Komiyama, Kanki; Ōmura, Satoshi. Structural study of isoflavonoids possessing antioxidant activity isolated from the fermentation broth of Streptomyces sp. J. Antibiot. 42(9), 1350-1355 (1989).

195. Kirst, H. A.; Willard, K. E.; Debono, M.; Toth, J. E.; Truedell, B. A.; Leeds, J. P.; Ott, J. L.; Felty-Elty-Duckworth, A. M.; Counter, F. T.; Ose, E. E.; Crouse, G. D.; Tustin, J. M.; Ōmura, Satoshi. Structure-activity studies of 20-deoxo-20-amino derivatives of tylosin-related macrolides. J. Antibiot. 42 (11), 1673-1683 (1989).

196. Tanaka, Haruo; Kawakami, Takatoshi; Yang, Zhi Bo; Komiyama, Kanki; Ōmura, Satoshi. Potentiation of cytotoxicity and antitumor activity of adenosine analogs by the adenosine deaminase inhibitor adecypenol. J. Antibiot. 42(11), 1722-1724 (1989).

197. Funayama, Shinji; Ishibashi, Masami; Anraku, Yumi; Miyauchi, Mayumi; Mori, Hiroharu; Komiyama, Kanki; Ōmura, Satoshi. Novel cytocidal antibiotics, glucopiericidinols $\mathrm{A}_{1}$ and $\mathrm{A}_{2}$. Taxonomy, fermentation, isolation, structure elucidation and biological characteristics. J. Antibiot. 42(12), 1734-1740 (1989).
198. Masuma, Rokuro; Zhen, Dezhong; Tanaka, Yoshitake; Ōmura, Satoshi. Site of regulation of nanaomycin biosynthesis by inorganic phosphate. J. Antibiot. 43(1), 83-87 (1990).

199. Komiyama, Kanki; Funayama, Shinji; Anraku, Yumi; Ishibashi, Masami; Takahashi, Yoko; Ōmura, Satoshi. Novel antibiotics, furaquinocins A and B. Taxonomy, fermentation, isolation, and physico-chemical and biological characteristics. J. Antibiot. 43(3), 247-252 (1990).

200. Kakinuma, Shizuko; Ikeda, Haruo; Ōmura, Satoshi; Hopwood, David A. Biosynthesis of kalafungin in Streptomyces tanashiensis. J. Antibiot. 43(4), 391-396 (1990).

201. Kumagai, Hidetoshi; Tomoda, Hiroshi; Ōmura, Satoshi. Method of search for microbial inhibitors of mevalonate biosynthesis using animal cells. J. Antibiot. 43(4), 397-402 (1990).

202. Ōmura, Satoshi; Tanaka, Yoshitake; Kanaya, Isao; Shinose, Mayumi; Takahashi, Yoko. Phthoxazolin, a specific inhibitor of cellulose biosynthesis, produced by a strain of Streptomyces sp. J. Antibiot. 43(8), 1034-1036 (1990).

203. Oshino, Kazushi; Kumagai, Hidetoshi; Tomoda, Hiroshi; Ōmura, Satoshi. Mechanism of action of atpenin B on Raji cells. J. Antibiot. 43(9), 1064-1068 (1990).

204. Tomoda, Hiroshi; Ōmura, Satoshi. New strategy for discovery of enzyme inhibitors: screening with intact mammalian cells or intact microorganisms having special functions. J. Antibiot. 43 (10), 1207-1222 (1990).

205. Mori, Hiroharu; Funayama, Shinji; Sudo, Yohei; Komiyama, Kanki; Ōmura, Satoshi. A new antibiotic, 13-hydroxyglucopiericidin A isolation, structure elucidation and biological characteristics. J. Antibiot. 43(10), 1329-1331 (1990).

206. Kumagai, Hidetoshi; Nishida, Hiroyuki; Imamura, Nobutaka; Tomoda, Hiroshi; Ōmura, Satoshi; Bordner, Jon. The structures of atpenins A4, A5 and B, new antifungal antibiotics produced by Penicillium sp. J. Antibiot. 43(12), 1553-1558 (1990).

207. Ōmura, Satoshi; Fujimoto, Tomoko; Otoguro, Kazuhiko; Matsuzaki, Keiichi; Moriguchi, Ryozo; Tanaka, Haruo; Sasaki, Yasuharu. Lactacystin, a novel microbial metabolite, induces neuritogenesis of neuroblastoma cells. J. Antibiot. 44(1), 113-116 (1991).

208. Ōmura, Satoshi; Matsuzaki, Keiichi; Fujimoto, Tomoko; Kosuge, Kazuhito; Furuya, Toshio; Fujita, Shigeo; Nakagawa, Akira. Structure of lactacystin, a new microbial metabolite which induces differentiation of neuroblastoma cells. J. Antibiot. 44 (1), 117-118 (1991).

209. Tomoda, Hiroshi; Nishida, Hiroyuki; Masuma, Rokuro; Cao, Jing; Okuda, Shigenobu; Ōmura, Satoshi. Purpactins, new inhibitors of acyl-CoA: cholesterol acyltransferase produced by Penicillium purpurogenum. I. Production, isolation and physico-chemical and biological properties. J. Antibiot. 44(2), 136-143 (1991).

210. Nishida, Hiroyuki; Tomoda, Hiroshi; Cao, Jing; Okuda, Shigenobu; Ōmura, Satoshi. Purpactins, new inhibitors of acyl-CoA: cholesterol acyltransferase produced by Penicillium purpurogenum. II. Structure elucidation of purpactins A, B and C. J. Antibiot. 44(2), 144-151 (1991).

211. Nishida, Hiroyuki; Tomoda, Hiroshi; Cao, Jing; Araki, Sachiko; Okuda, Shigenobu; Ōmura, Satoshi. Purpactins, new inhibitors of acyl-CoA: cholesterol acyltransferase produced by Penicillium purpurogenum. III. Chemical modification of purpactin A. J. Antibiot. 44(2), 152-159 (1991). 
212. Ishibashi, Masami; Funayama, Shinji; Anraku, Yumi; Komiyama, Kanki; Ōmura, Satoshi. Novel antibiotics, furaquinocins C, D, E, F, G and H. J. Antibiot. 44(4), 390-395 (1991).

213. Ōmura, Satoshi; Ikeda, Haruo; Tanaka, Haruo. Selective production of specific components of avermectins in Streptomyces avermitilis. J. Antibiot. 44(5), 560-563 (1991).

214. Tsuzuki, Kazuo; Yan, Fu Shan; Otoguro, Kazuhiko; Ōmura, Satoshi. Synthesis and nematocidal activities of jietacin A and its analogs. J. Antibiot. 44(7), 774-784 (1991).

215. Komiyama, Kanki; Funayama, Shinji; Anraku, Yumi; Ishibashi, Masami; Takahashi, Yoko; Kawakami, Takatoshi; Ōmura, Satoshi. A new antibiotic, okicenone. I. Taxonomy, fermentation, isolation and biological characteristics. J. Antibiot. 44(8), 814-818 (1991).

216. Funayama, Shinji; Ishibashi, Masami; Komiyama, Kanki; Ōmura, Satoshi. A new antibiotic, okicenone. II. Physico-chemical properties and structure elucidation. J. Antibiot. 44(8), 819-823 (1991).

217. Kakinuma, Shizuko; Takada, Yoshio; Ikeda, Haruo; Tanaka, Haruo; Ōmura, Satoshi; Hopwood, David A. Cloning of large DNA fragments, which hybridize with actinorhodin biosynthesis genes, from kalafungin and nanaomycin A methyl ester producers and identification of genes for kalafungin biosynthesis of the kalafungin producer. J. Antibiot. 44(9), 995-1005 (1991).

218. Kumagai, Hidetoshi; Tomoda, Hiroshi; Ōmura, Satoshi. Biosynthesis of antibiotic 1233A (F-244) and preparation of $\left[{ }^{14} \mathrm{C}\right] 1233$ A. J. Antibiot. 45(4), 563-7 (1992).

219. Sunazuka, Toshiaki; Tsuzuki, Kazuo; Kumagai, Hidetoshi; Tomoda, Hiroshi; Tanaka, Haruo; Nagashima, Hajime; Hashizume, Hirokazu; Ōmura, Satoshi. Synthesis of 1233A analogs and their inhibitory activity against hydroxymethylglutaryl coenzyme A synthase. J. Antibiot. 45(7), 1139-1147 (1992).

220. Tomoda, Hiroshi; Huang, Xin Hui; Nishida, Hiroyuki; Masuma, Rokuro; Kim, Young Kook; Ōmura, Satoshi. Glisoprenins, new inhibitors of acyl-CoA. cholesterol acyltransferase produced by Gliocladium sp. FO-1513. I. Production, isolation, and physicochemical and biological properties. J. Antibiot. 45(8), 1202-1206 (1992).

221. Tomoda, Hiroshi; Nishida, Hiroyuki; Huang, Xin Hui; Masuma, Rokuro; Kim, Young Kook; Ōmura, Satoshi. New cyclodepsipeptides, enniatins D, E, and F produced by Fusarium sp. FO-1305. J. Antibiot. 45(8), 1207-1215 (1992).

222. Tomoda, Hiroshi; Huang, Xin Hui; Cao, Jin; Nishida, Hiroyuki; Nagao, Ryuichi; Okuda, Shigenobu; Tanaka, Haruo; Ōmura, Satoshi; Arai, Hiroyuki; Inoue, Keizo. Inhibition of acyl-CoA: cholesterol acyltransferase activity by cyclodepsipeptide antibiotics. J. Antibiot. 45(10), 1626-1632 (1992).

223. Nishida, Hiroyuki; Huang, Xin-Hui; Tomoda, Hiroshi; Ōmura, Satoshi. Glisoprenins, new inhibitors of acyl-CoA: cholesterol acetyltransferase produced by Gliocladium sp. FO-1513. II. Structure elucidation of glisoprenins A and B. J. Antibiot. 45 (10), 1669-1676 (1992).

224. Funayama, Shinji; Nozoe, Shigeo; Tronquet, Claude; Anraku, Yumi; Komiyama, Kanki; Ōmura, Satoshi. Isolation and structure of a new polyether antibiotic, octacyclomycin. J. Antibiot. 45(10), 1686-1691 (1992).

225. Van der Pyl, Didier; Inokoshi, Junji; Shiomi, Kazuro; Yang, Hong; Takeshima, Hideo; Ōmura, Satoshi. Inhibition of farnesylprotein transferase by gliotoxin and acetylgliotoxin. J. Antibiot. 45(11), 1802-1805 (1992).
226. Ōmura, Satoshi; Van Der Pyl, Didier; Inokoshi, Junji; Takahashi, Yoko; Takeshima, Hideo. Pepticinnamins, new farnesyl-protein transferase inhibitors produced by an actinomycete. I. Producing strain, fermentation, isolation, and biological activity. J. Antibiot. 46(2), 222-228 (1993).

227. Shiomi, Kazuro; Yang, Hong; Inokoshi, Junji; Van Der Pyl, Didier; Nakagawa, Akira; Takeshima, Hideo; Ōmura, Satoshi. Pepticinnamins, new farnesyl-protein transferase inhibitors produced by an actinomycete. II. Structural elucidation of pepticinnamin E. J. Antibiot. 46(2), 229-234 (1993).

228. Tabata, Noriko; Suzumura, Yasuko; Tomoda, Hiroshi; Masuma, Rokuro; Haneda, Katsuji; Kishi, Masanori; Iwai, Yuzuru; Ōmura, Satoshi. Xanthoquinodins, new anticoccidial agents produced by Humicola sp.: production, isolation, and physico-chemical and biological properties. J. Antibiot. 46(5), 749-755 (1993).

229. Tabata, Noriko; Tomoda, Hiroshi; Takahashi, Yoko; Haneda, Katsuji; Iwai, Yuzuru; Woodruff, H. Boyd; Ōmura, Satoshi. Diolmycins, new anticoccidial agents produced by Streptomyces sp. I. Production, isolation, and physico-chemical and biological properties. J. Antibiot. 46(5), 756-761 (1993).

230. Tabata, Noriko; Sunazuka, Toshiaki; Tomoda, Hiroshi; Nagamitsu, Tohru; Iwai, Yuzuru; Ōmura, Satoshi. Diolmycins, new anticoccidial agents produced by Streptomyces sp. II. Structure elucidation of diolmycins A1, A2, B1 and B2, and synthesis of diolmycin A1. J. Antibiot. 46(5), 762-769 (1993).

231. Tomoda, Hiroshi; Kumagai, Hidetoshi; Tanaka, Haruo; Ōmura, Satoshi. Specific binding of $\beta$-lactone 1233A to 3-hydroxy-3methylglutaryl-coenzyme A synthase. J. Antibiot. 46(5), 872-874 (1993).

232. Ōmura, Satoshi; Tomoda, Hiroshi; Kim, Young Kook; Nishida, Hiroyuki. Pyripyropenes, highly potent inhibitors of acyl-CoA: cholesterol acyltransferase produced by Aspergillus fumigatus. J. Antibiot. 46(7), 1168-1169 (1993).

233. Sunazuka, Toshiaki; Tabata, Noriko; Nagamitsu, Tohru; Tomoda, Hiroshi; Ōmura, Satoshi. Synthesis of diolmycin analogs and their anticoccidial activities. J. Antibiot. 46(7), 1178-1180 (1993).

234. Tanaka, Yoshitake; Kanaya, Isao; Takahashi, Yoko; Shinose, Mayumi; Tanaka, Haruo; Ōmura, Satoshi. Phthoxazolin A, a specific inhibitor of cellulose biosynthesis from microbial origin. I. Discovery, taxonomy of producing microorganism, fermentation, and biological activity. J. Antibiot. 46(8), 1208-1213 (1993).

235. Tanaka, Yoshitake; Kanaya, Isao; Shiomi, Kazuro; Tanaka, Haruo; Ōmura, Satoshi. Phthoxazolin A, a specific inhibitor of cellulose biosynthesis from microbial origin. II. Isolation, physico-chemical properties, and structural elucidation. J. Antibiot. 46(8), 1214-1218 (1993).

236. Komiyama, Kanki; Takamatsu, Satoshi; Takahashi, Yoko; Shinose, Mayumi; Hayashi, Masahiko; Tanaka, Haruo; Iwai, Yuzuru; Ōmura, Satoshi; Imokawa, Genji. New inhibitors of melanogenesis, OH-3984 K1 and K2. I. Taxonomy, fermentation, isolation and biological characteristics. J. Antibiot. 46(10), 1520-1525 (1993).

237. Takamatsu, Satoshi; Rho, Mun Chual; Hayashi, Masahiko; Komiyama, Kanki; Tanaka, Haruo; Ōmura, Satoshi; Imokawa, Genji. New inhibitors of melanogenesis, OH-3984 K1 and K2. II. Physico-chemical properties and structural elucidation. J. Antibiot. 46(10), 1526-1529 (1993).

238. Komiyama, Kanki; Otoguro, Kazuhiko; Segawa, Toshiaki; Shiomi, Kazuro; Yang, Hong; Takahashi, Yoko; Hayashi, Masahiko; Otani, Toshio; Ōmura, Satoshi. A new antibiotic, 
cypemycin: taxonomy, fermentation, isolation and biological characteristics. J. Antibiot. 46(11), 1666-1671 (1993).

239. Tabata, Noriko; Tomoda, Hiroshi; Masuma, Rokuro; Haneda, Katsuji; Iwai, Yuzuru; Ōmura, Satoshi. Hynapenes A, B and C, new anticoccidial agents produced by Penicillium sp. I. Production, isolation and physico-chemical and biological properties. J. Antibiot. 46(12), 1849-1853 (1993).

240. Tabata, Noriko; Tomoda, Hiroshi; Iwai, Yuzuru; Ōmura, Satoshi. Hynapenes A, B and C, new anticoccidial agents produced by Penicillium sp. II. Structure elucidation. J. Antibiot. 46(12), 1854-1858 (1993).

241. Ōmura, Satoshi; Tanaka, Haruo; Matsuzaki, Keiichi; Ikeda, Hodaka; Masuma, Rokuro. Isochromophilones I and II, novel inhibitors against gp120-CD4 binding from Penicillium sp. J. Antibiot. 46(12), 1908-1911 (1993).

242. Masuma, Rokuro; Tabata, Noriko; Tomoda, Hiroshi; Haneda, Katsuji; Iwai, Yuzuru; Ōmura, Satoshi. Arohynapenes A and B, new anticoccidial agents produced by Penicillium sp. Taxonomy, fermentation, and structure elucidation. J. Antibiot. 47(1), 46-53 (1994).

243. Tomoda, Hiroshi; Kim, Young Kook; Nishida, Hiroyuki; Masuma, Rokuro; Ōmura, Satoshi. Pyripyropenes, novel inhibitors of acyl-CoA. cholesterol acyltransferase produced by Aspergillus fumigatus. I. Production, isolation, and biological properties. J. Antibiot. 47(2), 148-153 (1994).

244. Kim, Young Kook; Tomoda, Hiroshi; Nishida, Hiroyuki; Sunazuka, Toshiaki; Obata, Rika; Ōmura, Satoshi. Pyripyropenes, novel inhibitors of acyl-CoA: cholesterol acyltransferase produced by Aspergillus fumigatus. II. Structure elucidation of pyripyropenes A, B, C and D. J. Antibiot. 47(2), 154-162 (1994).

245. Haneda, Katuji; Shinose, Mayumi; Seino, Akio; Tabata, Noriko; Tomoda, Hiroshi; Iwai, Yuzuri; Ōmura, Satoshi. Cytosaminomycins, new anticoccidial agents produced by Streptomyces sp. KO-8119. I. Taxonomy, production, isolation and physico-chemical and biological properties. J. Antibiot. 47 (7), 774-781 (1994).

246. Shiomi, Kazuro; Haneda, Katsuji; Tomoda, Hiroshi; Iwai, Yuzuru; Ōmura, Satoshi. Cytosaminomycins, new anticoccidial agents produced by Streptomyces sp. KO-8119. II. Structure elucidation of cytosaminomycins A, B, C and D. J. Antibiot. 47 (7), 782-786 (1994).

247. Yoshida, Hiroshi; Arai, Noriko; Sugoh, Masako; Shiomi, Kazuro; Shinose, Mayumi; Tanaka, Yoshitake; Ōmura, Satoshi. 4-Chlorothreonine, a herbicidal antimetabolite produced by Streptomyces sp. OH-5093. J. Antibiot. 47(10), 1165-1166 (1994).

248. Matsuzaki, Keiichi; Ikeda, Hodaka; Ogino, Tomoaki; Matsumoto, Atsuko; Woodruff, H. Boyd; Tanaka, Haruo; Ōmura, Satoshi. Chloropeptins I and II, novel inhibitors against gp120-CD4 binding from Streptomyces sp. J. Antibiot. 47(10), 1173-1174 (1994).

249. Huang, Xin-Hui; Tomoda, Hiroshi; Nishida, Hiroyuki; Masuma, Rokuro; Ōmura, Satoshi. Terpendoles, novel ACAT inhibitors produced by Albophoma yamanashiensis. I. Production, isolation and biological properties. J. Antibiot. 48(1), 1-4 (1995).

250. Huang, Xin-Hui; Nishida, Hiroyuki; Tomoda, Hiroshi; Tabata, Noriko; Shiomi, Kazuro; Yang, Da-Jun; Takayanagi, Hiroaki; Ōmura, Satoshi. Terpendoles, novel ACAT inhibitors produced by Albophoma yamanashiensis. II. Structure elucidation of terpendoles A, B, C and D. J. Antibiot. 48(1), 5-11 (1995).

251. Tabata, Noriko; Tomoda, Hiroshi; Masuma, Rokuro; Iwai, Yuzuru; Ōmura, Satoshi. Fudecalone, a new anticoccidial agent produced by Penicillium sp. FO-2030. J. Antibiot. 48(1), 53-58 (1995).

252. Pang, Chang-Hong; Matsuzaki, Keiichi; Ikeda, Haruo; Tanaka, Haruo; Ōmura, Satoshi. Production of 6,8a-seco-6,8a-deoxy derivatives of avermectins by a mutant strain of Streptomyces avermitilis. J. Antibiot. 48(1), 59-66 (1995).

253. Tabata, Noriko; Tomoda, Hiroshi; Iwai, Yuzuru; Ōmura, Satoshi. Arohynapene D, a new anticoccidial agent produced by Penicillium sp. FO-2295. J. Antibiot. 48(1), 83-84 (1995).

254. Pang, Chang-Hong; Matsuzaki, Keiichi; Ikeda, Haruo; Tanaka, Haruo; Omura, Satoshi. Production of a new methylated 6,8a-seco-6,8a-deoxy derivative of the avermectins by a transformant strain of Streptomyces avermitilis. J. Antibiot. 48(1), 92-94 (1995).

255. Ikeda, Haruo; Takada, Yoshio; Pang, Chang-Hong; Matsuzaki, Keiichi; Tanaka, Haruo; Ōmura, Satoshi. Direct production of 5-oxo derivatives of avermectins by a recombinant strain of Streptomyces avermitilis. J. Antibiot. 48(1), 95-97 (1995).

256. Yamada, Haruki; Shiomi, Kazuro; Xu, Qi; Nagai, Takayuki; Shibata, Maki; Oya, Izumi; Takahashi, Yoko; Ōmura, Satoshi. New glycosidases inhibitors, panosialins $\mathrm{D}$ and $\mathrm{wD}$ produced by Streptomyces sp. OH-5186. J. Antibiot. 48(3), 205-209 (1995).

257. Katagiri, Masako; Hayashi, Masahiko; Matsuzaki, Keiichi; Tanaka, Haruo; Ōmura, Satoshi. The neuritogenesis inducer lactacystin arrests cell cycle at both G0/G1 and G2 phases in Neuro 2a cells. J. Antibiot. 48(4), 344-346 (1995).

258. Kakinuma, Shizuko; Ikeda, Harudo; Takada, Yoshio; Tanaka, Haruo; Hopwood, David A.; Ōmura, Satoshi. Production of the new antibiotic tetrahydrokalafungin by transformants of the kalafungin producer Streptomyce stanashiensis. J. Antibiot. 48(6), 484-487 (1995).

259. Tomoda, Hiroshi; Tabata, Noriko; Yang, Da-Jun; Takayanagi, Hiroaki; Nishida, Hiroyuki; Ōmura, Satoshi. Pyripyropenes, novel ACAT inhibitors produced by Aspergillus fumigatus. III. Structure elucidation of pyripyropenes E to L. J. Antibiot. 48(6), 495-503 (1995).

260. Ikeda, Haruo; Pang, Chang-Hong; Endo, Hirofumi; Ohta, Toshio; Tanaka, Haruo; Ōmura, Satoshi. Construction of a single component producer from the wild-type avermectin producer Streptomyces avermitilis. J. Antibiot. 48(6), 532-534 (1995).

261. Ōmura, Satoshi; Sasaki, Yasuharu; Iwai, Yuzuru; Takeshima, Hideo. Staurosporine, a potentially important gift from a microorganism. J. Antibiot. 48(7), 535-548 (1995).

262. Ikeda, Haruo; Ōmura, Satoshi. Control of avermectin biosynthesis in Streptomyces avermitilis for the selective production of a useful component. J. Antibiot. 48(7), 549-562 (1995).

263. Arai, Noriko; Shiomi, Kazuro; Tomoda, Hiroshi; Tabata, Noriko; Yang, Da Jun; Masuma, Rokuro; Kawakubo, Tomoya; Ōmura, Satoshi. Isochromophilones III-VI, inhibitors of acyl-CoA: cholesterol acyltransferase produced by Penicillium multicolor FO-3216. J. Antibiot. 48(7), 696-702 (1995).

264. Matsuzaki, Keiichi; Ikeda, Hodaka; Masuma, Rokuro; Tanaka, Haruo; Ōmura, Satoshi. Isochromophilones I and II, novel inhibitors against gp120-CD4 binding produced by Penicillium multicolor FO-2338. I. Screening, taxonomy, fermentation, isolation and biological activity. J. Antibiot. 48(7), 703-707 (1995).

265. Matsuzaki, Keiichi; Tanaka, Haruo; Ōmura, Satoshi. Isochromophilones I and II, novel inhibitors against gp120-CD4 binding 
produced by Penicillium multicolor FO-2338. II. Structure elucidation. J. Antibiot. 48(7), 708-713 (1995).

266. Shiomi, Kazuro; Arai, Noriko; Shinose, Mayumi; Takahashi, Yoko; Yoshida, Hiroshi; Iwabuchi, Jun; Tanaka, Yoshitake; Ōmura, Satoshi. New antibiotics phthoxazolins B, C and D produced by Streptomyces sp. KO-7888. J. Antibiot. 48(7), 714-719 (1995).

267. Tanaka, Yoshitake; Sugoh, Masako; Ji, Wei; Iwabuchi, Jun; Yoshida, Hiroshi; Ōmura, Satoshi. Screening method for cellulose biosynthesis inhibitors with herbicidal activity. J. Antibiot. 48(7), 720-724 (1995).

268. Ōmura, Satoshi; Kuno, Fumiyoshi; Otoguro, Kazuhiko; Sunazuka, Toshiaki; Shiomi, Kazuro; Masuma, Rokuro; Iwai, Yuzuru. Arisugacin, a novel land selective inhibitor of acetylcholinesterase from Penicillium sp. FO-4259. J. Antibiot. 48(7), 745-746 (1995).

269. Nagamitsu, Tohru; Sunazuka, Toshiaki; Stump, Heike; Obata, Rika; Arima, Shiho; Matsuzaki, Keiichi; Tanak, Haruo; Ōmura, Satoshi. Structure-activity relationships of lactacystin, the first non-protein neurotrophic factor. J. Antibiot. 48(7), 747-748 (1995).

270. Obata, Rika; Sunazuka, Toshiaki; Zhuorong, Li; Tomoda, Hiroshi; Ōmura, Satoshi. Structure-activity relationships of pyripyropenes fungal acyl-CoA: cholesterol acyltransferase inhibitors. J. Antibiot. 48(7), 749-750 (1995).

271. Tomoda, Hiroshi; Tabata, Noriko; Yang, Da-Jun; Takayanagi, Hiroaki; Ōmura, Satoshi. Terpendoles, novel ACAT inhibitors produced by Albophoma yamanashiensis. III. Production, isolation and structure elucidation of new components. J. Antibiot. 48 (8), 793-804 (1995).

272. Tomoda, Hiroshi; Ito, Minako; Tabata, Noriko; Masuma, Rokuro; Yamaguchi, Yuichi; Ōmura, Satoshi. Amidepsines, inhibitors of diacylglycerol acyltransferase produced by Humicola sp. FO-2942. I. Production, isolation and biological properties. J. Antibiot. 48(9), 937-941 (1995).

273. Tomoda, Hiroshi; Tabata, Noriko; Ito, Minako; Ōmura, Satoshi. Amidepsines, inhibitors of diacylglycerol acyltransferase produced by Humicola sp. FO-2942. II. Structure elucidation of amidepsines A, B and C. J. Antibiot. 48(9), 942-947 (1995).

274. Takahashi, Senji; Uchida, Kenichi; Nakagawa, Akira; Miyake, Yoko; Kainosho, Masatsune; Matsuzaki, Keiichi; Ōmura, Satoshi. Biosynthesis of lactacystin. J. Antibiot. 48(9), 1015-1020 (1995).

275. Komiyama, Kanki; Takamatsu, Satoshi; Kim, Yong-Pil; Matsumoto, Atsuko; Takahashi, Yoko; Hayashi, Masahiko; Woodruff, H. Boyd; Ōmura, Satoshi. Louisianins A, B, C and $\mathrm{D}$ : non-steroidal growth inhibitors of testosterone-responsive SC 115 cells. I. Taxonomy, fermentation, isolation and biological characteristics. J. Antibiot. 48(10), 1086-1089 (1995).

276. Takamatsu, Satoshi; Kim, Yong-Pil; Hayashi, Masahiko; Furuhata, Kimio; Takayanagi, Hiroaki; Komiyama, Kanki; Woodruff, H. Boyd; Ōmura, Satoshi. Louisianines A, B, C and D: non-steroidal growth inhibitors of testosterone-responsive SC 115 cells. II. Physico-chemical properties and structural elucidation. J. Antibiot. 48(10), 1090-1094 (1995).

277. Shiomi, Kazuro; Yang, Hong; Xu, Qi; Arai, Noriko; Namiki, Mikie; Hayashi, Masahiko; Inokoshi, Junji; Takeshima, Hideo; Masuma, Rokuro; Komiyama, Kanki; Ōmura, Satoshi. Phenopyrrozin, a new radical scavenger produced by Penidllium sp. FO-2047. J. Antibiot. 48(12), 1413-1418 (1995).

278. Hayashi, Masahiko; Kim, Yong-Pil; Hiraoka, Hidemi; Natori, Masahiko; Takamatsu, Satoshi; Kawakubo, Tomoya; Masuma,
Rokuro; Komiyama, Kanki; Ōmura, Satoshi. Macrosphelide, a novel inhibitor of cell-cell adhesion molecule. I. Taxonomy, fermentation, isolation and biological activities. J. Antibiot. 48 (12), 1435-1439 (1995).

279. Tanaka, Yoshitake; Sugoh, Masako; Yoshida, Hiroshi; Arai, Noriko; Shiomi, Kazuro; Matsumoto, Atsuko; Takahashi, Yoko; Ōmura, Satoshi. Hydranthomycin, a new agroactive antibiotic produced by Streptomyces sp. K93-5305. J. Antibiot. 48(12), 1525-1526 (1995).

280. Enomoto, Yumi; Shiomi, Kazuro; Hayashi, Masahiko; Masuma, Rokuro; Kawakubo, Tomoya; Tomosawa, Keiichi; Iwai, Yuzuru; Ōmura, Satoshi. Herquline B, a new platelet aggregation inhibitor produced by Penicillium herquei Fg-372. J. Antibiot. 49(1), 50-53 (1996).

281. Takamatsu, Satoshi; Kim, Yong-Pil; Hayashi, Masahiko; Hiraoka, Hidemi; Natori, Masahiko; Ko, Iyama, Kanki; Ōmura, Satoshi. Macrosphelide, a novel inhibitor of cell-cell adhesion molecule. II. Physicochemical properties and structural elucidation. J. Antibiot. 49(1), 95-98 (1996).

282. Yang, Da-Jun; Tomoda, Hiroshi; Tabata, Noriko; Masuma, Rokuro; Ōmura, Satoshi. New isochromophilones VII and VIII produced by Penicillium sp. FO-4164. J. Antibiot. 49(3), 223-229 (1996).

283. Tabata, Noriko; Tomoda, Hiroshi; Iwai, Yuzuru; Ōmura, Satoshi. Xanthoquinodin B3, a new anticoccidial agent produced by Humicola sp. FO-888. J. Antibiot. 49(3), 267-271 (1996).

284. Tomoda, Hiroshi; Tabata, Noriko; Yang, Da-Jun; Namatame, Ichiji; Tanaka, Haruo; Ōmura, Satoshi. Pyripyropenes, novel ACAT inhibitors produced by Aspergillus fumigatus IV. Structure elucidation of pyripyropenes M to R. J. Antibiot. 49(3), 292-298 (1996).

285. Ōmura, Satoshi; Inokoshi, Junji; Uchida, Ryuji; Shiomi, Kazuro; Masuma, Rokuro; Kawakubo, Tomoya; Tanaka, Haruo; Iwai, Yuzuru; Kosemura, Seiji; Yamamura, Shosuke. Andrastins A-C, new protein farnesyltransferase inhibitors produced by Penicillium sp. FO-3929. I. Producing strain, fermentation, isolation, and biological activities. J. Antibiot. 49(5), 414-417 (1996).

286. Uchida, Ryuji; Shiomi, Kazuro; Inokoshi, Junji; Sunazuka, Toshiaki; Tanaka, Haruo; Iwai, Yuzuru; Takayanagi, Hiroaki; Ōmura, Satoshi. Andrastins A-C, new protein farnesyltransferase inhibitors produced by Penidllium sp. FO-3929. II. Structure elucidation and biosynthesis. J. Antibiot. 49(5), 418-424 (1996).

287. Takamatsu, Satoshi; Kim, Yong-Pil; Hyashi, Masahiko; Komiyama, Kanki; Imokawa, Genji; Ōmura, Satoshi. A new inhibitor of melanogenesis, albocycline $\mathrm{K} 3$, produced by Streptomyces sp. OH-3984. J. Antibiot. 49(5), 485-486 (1996).

288. Hayashi, Masahiko; Kim, Yong-Pil; Takamatsu, Satoshi; Preeprame, Srisomporn; Komiya, Takamaro; Masuma, Rokuro; Tanaka, Haruo; Komiyama, Kanki; Ōmura, Satoshi. Chlovalicin, a new cytocidal antibiotic produced by Sporothrix sp. FO-4649. I. Taxonomy, fermentation, isolation and biological activities. J. Antibiot. 49(7), 631-634 (1996).

289. Takamatsu, Satoshi; Kim, Yong-Pil; Komiya, Takamaro; Sunazuka, Toshiaki; Hayashi, Masahiko; Tanaka, Haruo; Komiyama, Kanki; Ōmura, Satoshi. Chlovalicin, a new cytocidal antibiotic produced by Sporothrix sp. FO-4649. II. Physicochemical properties and structural elucidation. J. Antibiot. 49(7), 635-638 (1996).

290. Sun, Xue-Long; Takayanagi, Hiroaki; Matsuzaki, Keiichi; Tanaka, Haruo; Furuhata, Kimio; Ōmura, Satoshi. Synthesis and 
inhibitory activities of isochromophilone analogs against gp120CD4 binding. J. Antibiot. 49(7), 689-692 (1996).

291. Nakagawa, Akira; Ōmura, Satoshi. Biosynthesis of bioactive microbial metabolites and its application to the structural studies and production of hybrid compounds. J. Antibiot. 49(8), 717-741 (1996).

292. Kuno, Fumiyoshi; Otoguro, Kazuhiko; Shiomi, Kazuro; Iwai, Yuzuru; Ōmura, Satoshi. Arisugacins A and B, novel and selective acetylcholinesterase inhibitors from Penicillium sp. FO-4259. I. Screening, taxonomy, fermentation, isolation and biological activity. J. Antibiot. 49(8), 742-747 (1996).

293. Kuno, Fumiyoshi; Shiomi, Kazuro; Otoguro, Kazuhiko; Sunazuka, Toshiaki; Ōmura, Satoshi. Arisugacins A and B, novel and selective acetylcholinesterase inhibitors from Penicillium sp. FO-4259. II. Structure elucidation. J. Antibiot. 49(8), 748-751 (1996).

294. Funabashi, Yasunori; Maeshiba, Yoshihiro; Inatomi, Nobuhiro; Tanayama, shigeharu; Harada, Setsuo; Itoh, Zen; Ōmura, Satoshi. Bioactive metabolites of EM574 and EM523, erythromycin derivatives having strong gastrointestinal motor stimulating activity. J. Antibiot. 49(8), 794-801 (1996).

295. Funabashi, Yasujoro; Hakoda, Seiji; Inatomi, Nobuhiro; Koyama, Katsuo; Tanida, Seiichi; Harada, Setsuo; Itoh, Zen; Ōmura, Satoshi. Microbial conversion of EM574 and EM523, gastrointestinal motor stimulating agents. J. Antibiot. 49(8), 802-810 (1996).

296. Uchida, Ryuji; Shiomi, Kazuro; Sunazuka, Toshiaki; Inokoshi, Junji; Nishizawa, Ai; Hirose, Tomoyasu; Tanaka, Haruo; Iwai, Yuzuru; Ōmura, Satoshi. Kurasoins A and B, new protein farnesyltransferase inhibitors produced by Paecilomyces sp. FO-3684. II. Structure elucidation and total synthesis. J. Antibiot. 49(9), 886-889 (1996).

297. Tomoda, Hiroshi; Yamaguchi, Yuichi; Tabata, Noriko; Kobayashi, Tatsuya; Masuma, Rokuro; Tanaka, Haruo; Ōmura, Satoshi. Amidepsine E, an inhibitor of diacylglycerol acyltransferase produced by Humicola sp. FO-5969. J. Antibiot. 49(9), 929-931 (1996).

298. Uchida, Ryuji; Shiomi, Kazuro; Inokoshi, Junji; Masuma, Pokuro; Kawakubo, Tomoya; Tanaka, Haruo; Iwai, Yuzuru; Ōmura, Satoshi. Kurasoins A and B, new protein farnesyltransferase inhibitors produced by Paecilomyces sp. FO-3684. I. Producing strain, fermentation, isolation, and biological activities. J. Antibiot. 49(9), 932-934 (1996).

299. Sztaricskai, Ferenc; Dinya, Zoltan; Puskas, Maria M.; Batta, Gyula; Masuma, Rokuro; Ōmura, Satoshi. Semisynthetic modification of antibiotic lincomycin. J. Antibiot. 49(9), 941-943 (1996).

300. Tanaka, Yoshitake; Matsuzaki, Keiichi; Zhong, Chong-Lin; Yoshida, Hiroshi; Kawakubo, Tomoya; Masuma, Rokuro; Tanaka, Haruo; Ōmura, Satoshi. Dechlorogeodin and its new dihydro derivatives, fungal metabolites with herbicidal activity. J. Antibiot. 49(10), 1056-1059 (1996).

301. Yamada, Rintaro; Seto, Minoru; Sasaki, Yasuharu; Sunazuka, Toshiaki; Harigaya, Yoshihiro; Iwai, Yuzuru; Ōmura, Satoshi. Synthesis and anti-platelet aggregation activity of water-soluble staurosporine derivatives. J. Antibiot. 49(10), 1070-1072 (1996).

302. Hayashi, Masahiko; Kim, Yong-Pil; Takamatsu, Satoshi; Enomoto, Akiko; Shinose, Mayumi; Takahashi, Yoko; Tanaka, Haruo; Komiyama, Kanki; Ōmura, Satoshi. Madindoline, a novel inhibitor of IL-6 activity from Streptomyces sp. K93-0711.
I. Taxonomy, fermentation, isolation and biological activities. J. Antibiot. 49(11), 1091-1095 (1996).

303. Obata, Rika; Sunazuka, Toshiaki; Li, Zhuorong; Tian, Zhiming; Harigaya, Yoshihiro; Tabata, Noriko; Tomoda, Hiroshi; Ōmura, Satoshi. Chemical modification and structure-activity relationships of pyripyropenes. 1. Modification at the four hydroxyl groups. J. Antibiot. 49(11), 1133-1148 (1996).

304. Obata, Rika; Sunazuka, Toshiaki; Kato, Yumiko; Tomoda, Hiroshi; Harigaya, Yoshihiro; Ōmura, Satoshi. Chemical modification and structure-activity relationships of pyripyropenes. 2. 1,11-Cyclic analogs. J. Antibiot. 49(11), 1149-1156 (1996).

305. Uchida, Ryuji; Shiomi, Kazuro; Inokoshi, Junji; Tanaka, Haruo; Iwai, Yuzuru; Ōmura, Satoshi. Andrastin D, novel protein farnesyltransferase inhibitor produced by Penicillium sp. FO-3929. J. Antibiot. 49 (12), 1278-1280 (1996).

306. Tanaka, Haruo; Matsuzaki, Keiichi; Nakashima, Hideki; Ogino, Tomoaki; Matsumoto, Atsuko; Ikeda, Hodaka; Woodruff, H. Boyd; Ōmura, Satoshi. Chloropeptins, New anti-HIV antibiotics inhibiting gp120-CD4 binding from Streptomyces sp. I. Taxonomy, fermentation, isolation, and physico-chemical properties, and biological activities. J. Antibiot. 50(1), 58-65 (1997).

307. Matsuzaki, Keiichi; Ogino, Tomoaki; Sunazuka, Toshiaki; Tanaka, Haruo; Ōmura, Satoshi. Chloropeptins, new anti-HIV antibiotics inhibiting gp120-CD4 binding from Streptomyces sp. II. Structure elucidation of chloropeptin I. J. Antibiot. 50(1), 66-69 (1997).

308. Kim, Yong-Pil; Takamatsu, Satoshi; Hayashi, Masahiko; Komiyama, Kanki; Ōmura, Satoshi. Pyridindolols K1 and K2, new alkaloids from Streptomyces sp. K93-0711. J. Antibiot. 50(3), 189-193 (1997).

309. Tanaka, Yoshitake; Yoshida, Hiroshi; Enomoto, Yumi; Shiomi, Kazuro; Shinose, Mayumi; Takahashi, Yoko; Liu, Jing-Rong; Ōmura, Satoshi. Malolactomycins C and D, new 40-membered macrolides active against Botrytis. J. Antibiot. 50(3), 194-200 (1997).

310. Obata, Rika; Sunazuka, Toshiaki; Tian, Zhiming; Tomoda, Hiroshi; Harigaya, Yoshihiro; Ōmura, Satoshi. Chemical modification and structure-activity relationships of pyripyropenes. 3. Synthetic conversion of pyridine-pyrone moiety. J. Antibiot. 50(3), 229-236 (1997).

311. Sunazuka, Toshiaki; Zhi-Ming, Tian; Harigaya, Yoshihiro; Takamatsu, Satoshi; Hayashi, Masahiko; Komiyama, Kanki; Ōmura, Satoshi. Efficient chemical conversion of louisianine A to $\mathrm{C}$ and $\mathrm{D}$, the inhibitor of angiogenesis. J. Antibiot. 50(3), 274-275 (1997).

312. Sunazuka, Toshiaki; Hirose, Tomoyasu; Zhi-Ming, Tian; Uchida, Ryuji; Shiomi, Kazuro; Harigaya, Yoshihiro; Ōmura, Satoshi. Syntheses and absolute structures of novel protein farnesyltransferase inhibitors, kurasoins A and B. J. Antibiot. 50(5), 453-455 (1997).

313. Arai, Noriko; Shiomi, Kazuro; Takamatsu, Satoshi; Komiyama, Kanki; Shinose, Mayumi; Takahashi, Yoko; Tanaka, Yoshitake; Iwai, Yuzuru; Liu, Jing-Rong; Ōmura, Satoshi. Amphistin, a new melanogenesis inhibitor, produced by an actinomycete. J. Antibiot. 50(10), 808-814 (1997).

314. Sztaricskai, Ferenc; Dinya, Zoltan; Batta, Gyula; Mocsari, Aniko; Hollosi, Miklos; Majer, Zsuzsa; Masuma, Rokuro; Ōmura, Satoshi. Chemical synthesis and structural study of lincomycin sulfoxides and a sulfone. J. Antibiot. 50(10), 866-873 (1997). 
315. Takamatsu, Satoshi; Hiraoka, Hidemi; Kim, Yong-Pil; Hayashi, Masahiko; Natori, Masahiko; Komiyama, Kanki; Ōmura, Satoshi. Macrosphelides C and D, novel inhibitors of cell adhesion. J. Antibiot. 50(10), 878-880 (1997).

316. Fang, Fang; Ui, Hideaki; Shiomi, Kazuro; Masuma, Rokuro; Yamaguchi, Yuuichi; Zhang, Cheng Gang; Zhang, Xian Wu; Tanaka, Yoshitake; Ömura, Satoshi. Two new components of the aspochalasins produced by Aspergillus sp. J. Antibiot. 50(11), 919-925 (1997).

317. Takamatsu, Satoshi; Kim, Yong-Pil; Enomoto, Akiko; Hayashi, Masahiko; Tanaka, Haruo; Komiyama, Kanki; Ōmura, Satoshi. Madindolines, novel inhibitors of IL-6 activity from Streptomyces sp. K93-0711. II. Physico-chemical properties and structural elucidation. J. Antibiot. 50(12), 1069-1072 (1997).

318. Rho, Mun-Chual; Toyoshima, Mitsuhide; Hayashi, Masahiko; Uchida, Ryuji; Shiomi, Kazuro; Komiyama, Kanki; Ōmura, Satoshi. Enhancement of drug accumulation by andrastin A produced by Penicillium sp. FO-3929 in vincristine-resistant KB cells. J. Antibiot. 51(1), 68-72 (1998).

319. Tanaka, Yoshitake; Shiomi, Kazuro; Kamei, Kiseko; SugohHagino, Masako; Enomoto, Yumi; Fang, Fang; Yamaguchi, Yuuichi; Masuma, Rokuro; Zhang, Cheng Gang; Zhang, Xian Wu; Ōmura, Satoshi. Antimalarial activity of radicicol, heptelidic acid and other fungal metabolites. J. Antibiot. 51(2), 153-160 (1998).

320. Tanaka, Yoshitake; Fang, Fang; Zhang, Cheng Gang; Zhang, Xian Wu; Ōmura, Satoshi. Heme-dependent radical generation from antimalarial fungal metabolites, radicicol and heptelidic acid. J. Antibiot. 51(4), 451-453 (1998).

321. Tomoda, Hiroshi; Tabata, Noriko; Masuma, Rokuro; Si, Shu-Yi; Ōmura, Satoshi. Erabulenols, inhibitors of cholesteryl ester transfer protein produced by Penicillium sp. FO-5637. I. Production, isolation and biological properties. J. Antibiot. 51 (7), 618-623 (1998).

322. Tabata, Noriko; Tomoda, Hiroshi; Ōmura, Satoshi. Erabulenols, inhibitors of cholesteryl ester transfer protein produced by Penicillium sp. FO-5637, II. Structure elucidation of erabulenols A and B. J. Antibiot. 51(7), 624-628 (1998).

323. Matsuzaki, Keiichi; Tahara, Harumi; Inokoshi, Junji; Tanaka, Haruo; Masuma, Rokuro; Ōmura, Satoshi. New brominated and halogen-less derivatives and structure-activity relationship of azaphilones inhibiting gp120-CD4 binding. J. Antibiot. 51(11), 1004-1011 (1998).

324. Namatame, Ichiji; Tomoda, Hiroshi; Si, Shuyi; Yamaguchi, Yuichi; Masuma, Rokuro; Ōmura, Satoshi. Beauveriolides, specific inhibitors of lipid droplet formation in mouse macrophages, produced by Beauveria sp. FO-6979. J. Antibiot. 52(1), 1-6 (1999).

325. Namatame, Ichiji; Tomoda, Hiroshi; Tabata, Noriko; Si, Shuyi; Ōmura, Satoshi. Structure elucidation of fungal beauveriolide III, a novel inhibitor of lipid droplet formation in mouse macrophages. J. Antibiot. 52(1), 7-12 (1999).

326. Zhang, Hua; Tomoda, Hiroshi; Tabata, Noriko; Oohori, Masako; Shinose, Mayumi; Takahashi, Yoko; Ōmura, Satoshi. Zelkovamycin, a new cyclic peptide antibiotic from Streptomyces sp. K96-0670. I. Production, isolation and biological properties. J. Antibiot. 52(1), 29-33 (1999).

327. Tabata, Noriko; Tomoda, Hiroshi; Zhang, Hua; Uchida, Ryuji; Ōmura, Satoshi. Zelkovamycin, a new cyclic peptide antibiotic from Streptomyces sp. K96-0670. II. Structure elucidation. J. Antibiot. 52(1), 34-39 (1999).
328. Ōmura, Satoshi; Enomoto, Yumi; Shinose, Mayumi; Takahashi, Yoko; Iwai, Yuzuru; Shiomi, Kazuro. Isolation and structure of a new antibiotic viridomycin F produced by Streptomyces sp. K96-0188. J. Antibiot. 52(1), 61-64 (1999).

329. Sunazuka, Toshiaki; Takizawa, Hajime; Desaki, Masashi; Suzuki, Kunihiko; Obata, Rika; Otoguro, Kazuhiko; Ōmura, Satoshi. Effects of erythromycin and its derivatives on interleukin-8 release by human bronchial epithelial cell line BEAS-2B cells. J. Antibiot. 52(1), 71-74 (1999).

330. Tomoda, Hiroshi; Matsushima, Chikako; Tabata, Noriko; Namatame, Ichiji; Tanaka, Haruo; Bamberger, Mark J.; Arai, Hiroyuki; Fukazawa, Masayoshi; Inoue, Keizo; Ōmura, Satoshi. Structure-specific inhibition of cholesteryl ester transfer protein by azaphilones. J. Antibiot. 52(2), 160-170 (1999).

331. Fukami, Akiko; Taniguchi, Yosuke; Nakamura, Tomonori; Rho, Mun-Chual; Kawaguchi, Kanako; Hayashi, Masahiko; Komiyama, Kanki; Ōmura, Satoshi. New members of the macrosphelides from Microsphaeropsis sp. FO-5050 IV. J. Antibiot. 52(5), 501-504 (1999).

332. Uchida, Ryuji; Tomoda, Hiroshi; Dong, Yuesheng; Ōmura, Satoshi. Alutenusin, a specific neutral sphingomyelinase inhibitor, produced by Penicillium sp. FO-7436. J. Antibiot. 52 (6), 572-574 (1999).

333. Ōmura, Satoshi; Tomoda, Hiroshi; Tabata, Noriko; Ohyama, Yukako; Abe, Tatsuo; Namikoshi, Michio. Roselipins, novel fungal metabolites having a highly methylated fatty acid modified with a mannose and an arabinitol. J. Antibiot. 52(6), 586-589 (1999).

334. Tomoda, Hiroshi; Ohyama, Yukako; Abe, Tatsuo; Tabata, Noriko; Namikoshi, Michio; Yamaguchi, Yuichi; Masuma, Rokuro; Ōmura, Satoshi. Roselipins, inhibitors of diacylglycerol acyltransferase, produced by Gliocladium roseum KF-1040. J. Antibiot. 52(8), 689-694 (1999).

335. Matsumoto, Tsukasa; Ishiyama, Aki; Yamaguchi, Yuuichi; Masuma, Rokuro; Ui, Hideaki; Shiomi, Kazuro; Yamada, Haruki; Ōmura, Satoshi. Novel cyclopentanone derivatives pentenocins A and $\mathrm{B}$, with interleukin- $1 \beta$ converting enzyme inhibitory activity, produced by Trichoderma hamatum FO-6903. J. Antibiot. 52(8), 754-757 (1999),

336. Tabata, Noriko; Ohyama, Yukako; Tomoda, Hiroshi; Abe, Tatsuo; Namikoshi, Michio; Ōmura, Satoshi. Structure elucidation of roselipins, inhibitors of diacylglycerol acyltransferase produced by Gliocladium roseum KF-1040. J. Antibiot. 52(9), 815-826 (1999).

337. Tomoda, Hiroshi; Namatame, Ichiji; Si, Shuyi; Kawaguchi, Kotaro; Masuma, Rokuro; Namikoshi, Michio; Ömura, Satoshi. Phenochalasins, inhibitors of lipid droplet formation in mouse macrophages, produced by Phomopsis sp. FT-0211. J. Antibiot. 52 (10), 851-856 (1999).

338. Tomoda, Hiroshi; Namatame, Ichiji; Tabata, Noriko; Kawaguchi, Kotaro; Si, Shuyi; Ōmura, Satoshi. Structure elucidation of fungal phenochalasins, novel inhibitors of lipid droplet formation in mouse macrophages. J. Antibiot. 52(10), 857-861 (1999).

339. Tanaka, Yoshitake; Kamei, Kiseko; Otoguro, Kazuhiko; Ōmura, Satoshi. Heme-dependent radical generation. possible involvement in antimalarial action of non-peroxide microbial metabolites, nanomycin A and radicicol. J. Antibiot. 52(10), 880-888 (1999).

340. Tabata, Noriko; Tomoda, Hiroshi; Yamaguchi, Yuichi; Masuma, Rokuro; Bamberger, Mark J.; Ōmura, Satoshi. Inhibition of 
cholesteryl ester transfer protein by fungal metabolites, L681,512. J. Antibiot. 52(11), 1042-1045 (1999).

341. Sztaricskai, Ferenc; Batta, Gyula; Dinya, Zoltan; Hornyak, Miklos; Roth, Erzsebet; Masuma, Rokuro; Ōmura, Satoshi. Structural modification of the lincomycin antibiotic. J. Antibiot. 52(11), 1050-1055 (1999).

342. Inokoshi, Junji; Shiomi, Kazuro; Masuma, Rokuro; Tanaka, Haruo; Yamada, Haruki; Ōmura, Satoshi. Funalenone, a novel collagenase inhibitor produced by Aspergillus niger. J. Antibiot. 52 (12), 1095-1100 (1999).

343. Tomoda, Hiroshi; Tabata, Noriko; Shinose, Mayumi; Takahashi, Yoko; Woodruff, H. Boyd; Ōmura, Satoshi. Ferroverdins, inhibitors of cholesteryl ester transfer protein produced by Streptomyces sp. WK-5344. I. Production, isolation and biological properties. J. Antibiot. 52(12), 1101-1107 (1999).

344. Tabata, Noriko; Tomoda, Hiroshi; Ōmura, Satoshi. Ferroverdins, inhibitors of cholesteryl ester transfer protein produced by Streptomyces sp. WK-5344. II. Structure elucidation. J. Antibiot. 52(12), 1108-1113 (1999).

345. Namatame, Ichiji; Tomoda, Hiroshi; Arai, Masayoshi; Ōmura, Satoshi. Effect of fungal metabolites cytochalasans on lipid droplet formation in mouse macrophages. J. Antibiot. 53(1), 19-25 (2000).

346. Otoguro, Kazuhiko; Shiomi, Kazuro; Yamaguchi, Yuuichi; Arai, Noriko; Sunazuka, Toshiaki; Masuma, Rokuro; Iwai, Yuzuru; Ōmura, Satoshi. Arisugacins C and D, novel acetylcholinesterase inhibitors and their related novel metabolites produced by Penicillium sp. FO-4259-11. J. Antibiot. 53(1), 50-57 (2000).

347. Obata, Rika; Sunazuka, Toshiaki; Harigaya, Yoshihiro; Rho, Mun-Chual; Tomoda, Hiroshi; Ōmura, Satoshi. Structure-activity relationships study of pyripyropenes: reversal of cancer cell multidrug resistance. J. Antibiot. 53(4), 422-425 (2000).

348. Li, Zhuorong; Sunazuka, Toshiaki; Yamada, Rintaro; Kato, Yumiko; Enomoto, Akiko; Hayashi, Masahiko; Harigaya, Yoshihiro; Ōmura, Satoshi. Synthesis and antiangiogenic activity of staurosporine derivatives. J. Antibiot. 53(4), 426-429 (2000).

349. Ōmura, Satoshi; Arai, Noriko; Yamaguchi, Yuuichi; Masuma, Rokuro; Iwai, Yuzuru; Namikoshi, Michio; Turberg, Andreas; Kolbl, Heinz; Shiomi, Kazuro. Argifin, a new chitinase inhibitor, produced by Gliocladium sp. FTD-0668. I. Taxonomy, fermentation, and biological activities. J. Antibiot. 53(6), 603-608 (2000).

350. Arai, Noriko; Shiomi, Kazuro; Iwai, Yuzuru; Ōmura, Satoshi. Argifin, a new chitinase inhibitor, produced by Gliocladium sp. FTD-0668. II. Isolation, physico-chemical properties, and structure elucidation. J. Antibiot. 53(6), 609-614 (2000).

351. Rho, Mun-Chual; Hayashi, Masahiko; Fukami, Akiko; Obata, Rika; Sunazuka, Toshiaki; Tomoda, Hiroshi; Komiyama, Kanki; Ōmura, Satoshi. Reversal of multidrug resistance by 7-O-benzoylpyripyropene A in multidrug-resistant tumor cells. J. Antibiot. 53(10), 1201-1206 (2000).

352. Fukami, Akiko; Nakamura, Tomonori; Kawaguchi, Kanako; Rho, Mun-Chual; Matsumoto, Atsuko; Takahashi, Yoko; Shiomi, Kazuro; Hayashi, Masahiko; Komiyama, Kanki; Ōmura, Satoshi. A new antimicrobial antibiotic from Actinoplanescapillaceus sp. K95-5561 ${ }^{\mathrm{T}}$. J. Antibiot. 53(10), 1212-1214 (2000).

353. Fukami, Akiko; Nakamura, Tomonori; Kim, Yong-Pil; Shiomi, Kazuro; Hayashi, Masahiko; Nagai, Takayuki; Yamada, Haruki; Komiyama, Kanki; Ōmura, Satoshi. A new anti-influenza virus antibiotic, 10-norparvulenone from Microsphaeropsis sp. FO-5050. J. Antibiot. 53(10), 1215-1218 (2000).
354. Oohori, Masako; Otoguro, Kazuhiko; Sunazuka, Toshiaki; Kunihiko, Suzuki; Iwai, Yuzuru; Ōmura, Satoshi. Effect of 14-membered ring macrolide compounds on rat leucocyte chemotaxis and the structure-activity relationships. J. Antibiot. 53(10), 1219-1222 (2000).

355. Ui, Hideaki; Shiomi, Kazuro; Yamaguchi, Yuuichi; Masuma, Rokuro; Nagamitsu, Tohru; Takano, Daisuke; Sunazuka, Toshiaki; Namikoshi, Michio; Ōmura, Satoshi. Nafuredin, a novel inhibitor of NADH-fumarate reductase, produced by Aspergillus niger FT-0554. J. Antibiot. 54(3), 234-238 (2001).

356. Enomoto, Yumi; Shiomi, Kazuro; Matsumoto, Atsuko; Takahashi, Yoko; Iwai, Yuzuru; Harder, Achim; Kolbl, Heinz; Woodruff, H. Boyd; Ōmura, Satoshi. Isolation of a new antibiotic oligomycin G produced by Streptomyces sp. WK-6150. J. Antibiot. 54(3), 308-313 (2001).

357. Handa, Masaki; Sunazuka, Toshiaki; Nagai, Kenichiro; Kumura, Ryouko; Shirahata, Tatsuya; Tian, Zhi-Ming; Otoguro, Kazuhiko; Harigaya, Yoshihiro; Ōmura, Satoshi. Convergent synthesis of arisugacin skeletons and their acetylcholinesterase inhibitory activity. J. Antibiot. 54(4), 382-385 (2001).

358. Handa, Masaki; Sunazuka, Toshiaki; Nagai, Kenichiro; Kimura, Ryouko; Otoguro, Kazuhiko; Harigaya, Yoshihiro; Ōmura, Satoshi. Determination of absolute stereochemistries of arisugacin $\mathrm{F}$ and territrem $\mathrm{B}$, novel acetylcholinesterase inhibitors. J. Antibiot. 54(4), 386-391 (2001).

359. Arai, Masayoshi; Tomoda, Hiroshi; Matsumoto, Atsuko; Takahashi, Yoko; Woodruff, Body H.; Ishiguro, Nobiko; Kobayashi, Susumu; Ōmura, Satoshi. Deacetylravidomycin M, a new inhibitor of IL-4 signal transduction, produced by Streptomyces sp. WK-6326. I. Taxonomy, fermentation, isolation and biological activities. J. Antibiot. 54(7), 554-561 (2001).

360. Arai, Masayoshi; Tomoda, Hiroshi; Tabata, Noriko; Ishiguro, Nobiko; Kobayashi, Susumu; Ōmura, Satoshi. Deacetylravidomycin M, a new inhibitor of IL-4 signal transduction, produced by Streptomyces sp. WK-6326. II. Structure elucidation. J. Antibiot. 54(7), 562-566 (2001),

361. Zhang, Hua; Tomoda, Hiroshi; Tabata, Noriko; Miura, Hiromi; Namikoshi, Michio; Yamaguchi, Yuichi; Masuma, Rokuro; Ōmura, Satoshi. Cladospolide D, a new 12-membered macrolide antibiotic produced by Cladosporium sp. FT-0012. J. Antibiot. 54 (8), 635-641 (2001).

362. Otoguro, Kazuhiko; Kohana, Akiko; Manabe, Chizu; Ishiyama, Aki; Ui, Hideaki; Shiomi, Kazuro; Yamada, Haruki; Ōmura, Satoshi. Potent antimalarial activities of polyether antibiotic, X-206. J. Antibiot. 54(8), 658-663 (2001).

363. Chiba, Harumi; Asanuma, Satoshi; Okamoto, Megumi; Inokoshi, Junji; Tanaka, Haruo; Fujita, Kazunobu; Ōmura, Satoshi. A simple screening system for anti-HIV drugs: syncytium formation assay using T-cell line tropic and macrophage tropic HIV env expressing cell lines-establishment and validation. J. Antibiot. 54(10), 818-826 (2001).

364. Uchida, Ryuji; Tomoda, Hiroshi; Arai, Masayoshi; Ōmura, Satoshi. Chlorogentisylquinone, a new neutral sphingomyelinase inhibitor, produced by a marine fungus. J. Antibiot. 54(11), 882-889 (2001).

365. Arai, Masayoshi; Tomoda, Hiroshi; Okuda, Takako; Wang, Haiyan; Tabata, Noriko; Masuma, Rokuro; Yamaguchi, Yuichi; Ōmura, Satoshi. Funicone-related compounds, potentiators of antifungal miconazole activity, produced by Talaromycesflavus FKI-0076. J. Antibiot. 55(2), 172-180 (2002). 
366. Otoguro, Kazuhiko; Ishiyama, Aki; Ui, Hideaki; Kobayashi, Miyuki; Manabe, Chizu; Yan, Guan; Takahashi, Yoko; Tanaka, Haruo; Yamada, Haruki; Ōmura, Satoshi. In vitro and in vivo antimalarial activities of the monoglycoside polyether antibiotic, K-41 against drug resistant strains of Plasmodia. J. Antibiot. 55 (9), 832-834 (2002).

367. Shiomi, Kazuro; Hatae, Kenji; Yamaguchi, Yuichi; Masuma, Rokuro; Tomoda, Hiroshi; Kobayashi, Susumu; Ōmura, Satoshi. New antibiotics miyakamides produced by a fungus. J. Antibiot. 55(11), 952-961 (2002).

368. Namatame, Ichiji; Matsuda, Daisuke; Tomoda, Hiroshi; Yamaguchi, Yuichi; Masuma, Rokuro; Kobayashi, Susumu; Ōmura, Satoshi. Selective production of fungal beauveriolide I or III by fermentation in amino acid-supplemented media. J. Antibiot. 55(12), 1048-1052 (2002).

369. Tomoda, Hiroshi; Tabata, Noriko; Ohyama, Yukako; Ōmura, Satoshi. Core structure in roselipins essential for eliciting inhibitory activity against diacylglycerol acyltransferase. J. Antibiot. 56(1), 24-29 (2003).

370. Otoguro, Kazuhiko; Ui, Hideaki; Ishiyama, Aki; Arai, Noriko; Kobayashi, Miyuki; Takahashi, Yoko; Masuma, Rokuro; Shiomi, Kazuro; Yamada, Haruki; Ōmura, Satoshi. In vitro antimalarial activities of the microbial metabolites. J. Antibiot. 56(3), 322-324 (2003).

371. Kim, Yong-Pil; Tomoda, Hiroshi; Iizima, Kousuke; Fukuda, Takashi; Matsumoto, Atsuko; Takahashi, Yoko; Ömura, Satoshi. Takanawaenes, novel antifungal antibiotics produced by Streptomyces sp. K99-5278. I. Taxonomy, fermentation, isolation and biological properties. J. Antibiot. 56(5), 448-453 (2003).

372. Fukuda, Takashi; Kim, Yong-Pil; Iizima, Kousuke; Tomoda, Hiroshi; Ōmura, Satoshi. Takanawaenes, novel antifungal antibiotics produced by Streptomyces sp. K99-5278. II. Structure elucidation. J. Antibiot. 56(5), 454-458 (2003).

373. Arai, Masayoshi; Yamamoto, Kazunobu; Namatame, Ichiji; Tomoda, Hiroshi; Ōmura, Satoshi. New monordens produced by amidepsine-producing fungus Humicola sp. FO-2942. J. Antibiot. 56(6), 526-532 (2003).

374. Yamamoto, Kazunobu; Hatano, Hiroko; Arai, Masayoshi; Shiomi, Kazuro; Tomoda, Hiroshi; Ōmura, Satoshi. Structure elucidation of new monordens produced by Humicola sp. FO-2942. J. Antibiot. 56(6), 533-538 (2003).

375. Sunazuka, Toshiaki; Yoshida, Kiminari; Oohori, Masako; Otoguro, Kazuhiko; Harigaya, Yoshihiro; Iwai, Yuzuru; Akagawa, Kiyoko S.; Ōmura, Satoshi. Effect of 14-membered macrolide compounds on monocyte to macrophage differentiation. J. Antibiot. 56(8), 721-724 (2003).

376. Otoguro, Kazuhiko; Ui, Hideaki; Ishiyama, Aki; Kobayashi, Miyuki; togashi, Hideaki; Takahashi, Yoko; Masuma, Rokuro; Tanaka, Haruo; Tomoda, Hiroshi; Yamada, Haruki; Ōmura, Satoshi. In vitro and in vivo antimalarial activities of a non-glycosidic 18-membered macrolide antibiotic, borrelidin, against drug-resistant strains of Plasmodia. J. Antibiot. 56(8), 727-729 (2003).

377. Handa, Masaki; Sunazuka, Toshiaki; Sugawara, Akihiro; Harigaya, Yoshihiro; Otoguro, Kazuhiko; Ōmura, Satoshi. Total syntheses of the AChE inhibitors ( \pm )-arisugacins $F$ and $G$. J. Antibiot. 56(8), 730-733 (2003).

378. Sakano, Yuichi; Shibuya, Masaaki; Matsumoto, Atsuko; Takahashi, Yoko; Tomoda, Hiroshi; Ōmura, Satoshi; Ebizuka, Yutaka. Lanopylins $A_{1}, B_{1}, A_{2}$ and $B_{2}$, novel lanosterol synthase inhibitors from Streptomyces sp. K99-5041. J. Antibiot. 56(10), 817-826 (2003).

379. Matsuda, Daisuke; Namatame, Ichiji; Tomoda, Hiroshi; Kobayashi, Susumu; Zocher, Rainer; Kleinkauf, Horst; Ōmura, Satoshi. New beauveriolides produced by amino acid-supplemented fermentation of Beauveria sp. FO-6979. J. Antibiot. 57(1), 1-9 (2004).

380. Fukuda, Takashi; Arai, Masayoshi; Yamaguchi, Yuichi; Masuma, Rokurou; Tomoda, Hiroshi; Ōmura, Satoshi. New beauvericins, potentiators of antifungal miconazole activity, produced by Beauveria sp. FKI-1366. I. Taxonomy, fermentation, isolation and biological properties. J. Antibiot. 57(2), 110-116 (2004).

381. Fukuda, Takashi; Arai, Masayoshi; Tomoda, Hiroshi; Ōmura, Satoshi. New beauvericins, potentiators of antifungal miconazole activity, produced by Beauveria sp. FKI-1366. II. Structure elucidation. J. Antibiot. 57(2), 117-124 (2004).

382. Otoguro, Kazuhiko; Ishiyama, Aki; Kobayashi, Miyuki; Sekiguchi, Hitomi; Izuhara, Takashi; Sunazuka, Toshiaki; Tomoda, Hiroshi; Yamada, Haruki; Ōmura, Satoshi. In vitro and in vivo antimalarial activities of a carbohydrate antibiotic, prumycin, against drug-resistant strains of Plasmodia. J. Antibiot. 57(6), 400-402 (2004).

383. Koizumi, Yukio; Arai, Masayoshi; Tomoda, Hiroshi; Ōmura, Satoshi. Fungerin, a fungal alkaloid, arrests the cell cycle in $\mathrm{M}$ phase by inhibition of microtubule polymerization. J. Antibiot. 57(7), 415-420 (2004).

384. Sakano, Yuichi; Shibuya, Masaaki; Yamaguchi, Yuichi; Masuma, Rokuro; Tomoda, Hiroshi; Ōmura, Satoshi; Ebizuka, Yutaka. Epohelmins A and B, novel lanosterol synthase inhibitors from a fungal strain FKI-0929. J. Antibiot. 57(9), 564-568 (2004).

385. Arai, Masayoshi; Koizumi, Yukio; Sato, Hitoshi; Kawabe, Takumi; Suganuma, Masashi; Kobayashi, Hidetaka; Tomoda, Hiroshi; Ōmura, Satoshi. Boromycin abrogates bleomycininduced G2 checkpoint. J. Antibiot. 57(10), 662-668 (2004).

386. Shiomi, Kazuro; Ui, Hideaki; Suzuki, Hideaki; Hatano, Hiroko; Nagamitsu, Tohru; Takano, Daisuke; Miyadera, Hiroko; Yamashita, Tetsuo; Kita, Kiyoshi; Miyoshi, Hideto; Harder, Achim; Tomoda, Hiroshi; Ōmura, Satoshi. A $\gamma$-lactone form nafuredin, nafuredin- $\gamma$, also inhibits helminth complex I. J. Antibiot. 58(1), 50-55 (2005).

387. Shiomi, Kazuro; Matsui, Ryosuke; Isozaki, Miki; Chiba, Harumi; Sugai, Takahiro; Yamaguchi, Yuichi; Masuma, Rokuro; Tomoda, Hiroshi; Chiba, Tomoko; Yan, Hua; Kitamura, Yoshihiro; Sugiura, Wataru; Ōmura, Satoshi; Tanaka, Haruo. Fungal phenalenones inhibit HIV-1 integrase. J. Antibiot. 58(1), 65-68 (2005).

388. Shiomi, Kazuro; Hatae, Kenji; Hatano, Hiroko; Matsumoto, Atsuko; Takahashi, Yoko; Jiang, Cheng-Lin; Tomoda, Hiroshi; Kobayashi, Susumu; Tanaka, Haruo; Ōmura, Satoshi. A new antibiotic, antimycin A9, produced by Streptomyces sp. K01-0031. J. Antibiot. 58(1), 74-78 (2005).

389. Yoshida, Kiminari; Sunazuka, Toshiaki; Nagai, Kenichiro; Sugawara, Akihiro; Cho, Achim; Nagamitsu, Tohru; Harigaya, Yoshihiro; Otoguro, Kazuhiko; Akagawa, Kiyoko S.; Ōmura, Satoshi. Macrolides with promotive activity of monocyte to macrophage differentiation. J. Antibiot. 58(1), 79-81 (2005).

390. Fukuda, Takashi; Matsumoto, Atsuko; Takahashi, Yoko; Tomoda, Hiroshi; Ōmura, Satoshi. Phenatic acids A and B, new potentiators of antifungal miconazole activity produced by Streptomyces sp. K03-0132. J. Antibiot. 58(4), 252-259 (2005). 
391. Fukuda, Takashi; Yamaguchi, Yuichi; Masuma, Rokuro; Tomoda, Hiroshi; Ōmura, Satoshi. Citridones, new potentiators of antifungal miconazole activity, produced by Penicillium sp. FKI-1938. I. Taxonomy, fermentation, isolation and biological properties. J. Antibiot. 58(5), 309-314 (2005).

392. Fukuda, Takashi; Tomoda, Hiroshi; Ōmura, Satoshi. Citridones, new potentiators of antifungal miconazole activity, produced by Penicillium sp. FKI-1938. II. Structure elucidation. J. Antibiot. 58 (5), 315-321 (2005).

393. Koyama, Nobuhiro; Nagahiro, Tomoko; Yamaguchi, Yuichi; Ohshiro, Taichi; Masuma, Rokuro; Tomoda, Hiroshi; Ōmura, Satoshi. Spylidone, a novel inhibitor of lipid droplet accumulation in mouse macrophages produced by Phoma sp. FKI-1840. J. Antibiot. 58(5), 338-345 (2005).

394. Uchida, Ryuji; Imasato, Rie; Yamaguchi, Yuichi; Masuma, Rokuro; Shiomi, Kazuro; Tomoda, Hiroshi; Ōmura, Satoshi. New sesquicillins, insecticidal antibiotics produced by Albophoma sp. FKI-1778. J. Antibiot. 58(6), 397-404 (2005).

395. Ui, Hideaki; Asanuma, Satoshi; Chiba, Harumi; Takahashi, Atsushi; Yamaguchi, Yuichi; Masuma, Rokuro; Ōmura, Satoshi; Tanaka, Haruo. Mycophenolic acid inhibits syncytium formation accompanied by reduction of gp120 expression. J. Antibiot. 58 (8), 514-518 (2005).

396. Shibuya, Masaaki; Snider, Barry B.; Sakano, Yuichi; Tomoda, Hiroshi; Ömura, Satoshi; Ebizuka, Yutaka. Revised structures of epohelmins A and B isolated as lanosterol synthase inhibitors from a fungal strain FKI-0929. J. Antibiot. 58(9), 599-601 (2005).

397. Koyama, Nobuhiro; Nagahiro, Tomoko; Yamaguchi, Yuichi; Masuma, Rokuro; Tomoda, Hiroshi; Ōmura, Satoshi. Stemphones, novel potentiators of imipenem activity against methicillin-resistant Staphylococcus aureus, produced by Aspergillus sp. FKI-2136. J. Antibiot. 58(11), 695-703 (2005).

398. Uchida, Ryuji; Imasato, Rie; Yamaguchi, Yuichi; Masuma, Rokuro; Shiomi, Kazuro; Tomoda, Hiroshi; Ōmura, Satoshi. New insecticidal antibiotics, hydroxyfungerins $A$ and B, produced by Metarhizium sp. FKI-1079. J. Antibiot. 58 (12), 804-809 (2005).

399. Uchida, Ryuji; Kim, Yong-Pil; Namatame, Ichiji; Tomoda, Hiroshi; Ōmura, Satoshi. Sespendole, a new inhibitor of lipid droplet synthesis in macrophages, produced by Pseudobotrytis terrestris FKA-25. J. Antibiot. 59(2), 93-97 (2006).

400. Banskota, Arjun H.; McAlpine, James B.; Sorensen, Dan; Aouidate, Mustapha; Piraee, Mahmood; Alarco, Anne-Marie; Ōmura, Satoshi; Shiomi, Kazuro; Farnet, Chris M.; Zazopoulos, Emmanuel. Isolation and identification of three new 5-alkenyl3,3 (2H)-furanones from two Streptomyces species using a genomic screening approach. J. Antibiot. 59(3), 168-176 (2006).

401. Uchida, Ryuji; Tomoda, Hiroshi; Ōmura, Satoshi. Biosynthesis of sespendole. J. Antibiot. 59(5), 298-302 (2006).

402. Uchida, Ryuji; Kim, Yong-Pil; Nagamitsu, Tohru; Tomoda, Hiroshi; Ōmura, Satoshi. Structure elucidation of fungal sespendole, an inhibitor of lipid droplet synthesis in macrophages. J. Antibiot. 59(6), 338-344 (2006).

403. Cane, David E.; He, Xiaofei; Kobayashi, Seiji; Ōmura, Satoshi; Ikeda, Haruo. Geosmin biosynthesis in Streptomyces avermitilis. Molecular cloning, expression, and mechanistic study of the germacradienol/geosmin synthase. J. Antibiot. 59(8), 471-479 (2006).

404. Fukuda, Takashi; Hasegawa, Yoko; Hagimori, Keiichi; Yamaguchi, Yuichi; Masuma, Rokuro; Tomoda, Hiroshi; Ōmura, Satoshi. Tensidols, new potentiators of antifungal miconazole activity, produced by Aspergillus niger FKI-2342. J. Antibiot. 59 (8), 480-485 (2006).

405. Ui, Hideaki; Shiomi, Kazuro; Suzuki, Hideaki; Hatano, Hiroko; Morimoto, Hiromi; Yamaguchi, Yuichi; Masuma, Rokuro; Sakamoto, Kimitoshi; Kita, Kiyoshi; Miyoshi, Hideto; Tomoda, Hiroshi; Tanaka, Haruo; Ōmura, Satoshi. Paecilaminol, a new $\mathrm{NADH}$-fumarate reductase inhibitor, produced by Paecilomyces sp. FKI-0550. J. Antibiot. 59(9), 591-596 (2006).

406. Uchida, Ryuji; Imasato, Rie; Yamaguchi, Yuichi; Masuma, Rokuro; Shiomi, Kazuro; Tomoda, Hiroshi; Ōmura, Satoshi. Yaequinolones, new insecticidal antibiotics produced by Penicillium sp. FKI-2140. I. Taxonomy, fermentation, isolation and biological activity. J. Antibiot. 59(10), 646-651 (2006).

407. Uchida, Ryuji; Imasato, Rie; Tomoda, Hiroshi; Ōmura, Satoshi. Yaequinolones, new insecticidal antibiotics produced by Penicillium sp. FKI-2140. II. Structural elucidation. J. Antibiot. 59(10), 652-658 (2006).

408. Ui, Hideaki; Shiomi, Kazuro; Suzuki, Hideaki; Hatano, Hiroko; Morimoto, Hiromi; Yamaguchi, Yuichi; Masuma, Rokuro; Sunazuka, Toshiaki; Shimamura, Hiroyuki; Sakamoto, Kimitoshi; Kita, Kiyoshi; Miyoshi, Hideto; Tomoda, Hiroshi; Ōmura, Satoshi. Verticipyrone, a new NADH-fumarate reductase inhibitor, produced by Verticillium sp. FKI-1083. J. Antibiot. 59 (12), 785-790 (2006).

409. Iwasaki, Shigeo; Ōmura, Satoshi. Search for protein farnesyltransferase inhibitors of microbial origin: our strategy and results as well as the results obtained by other groups. J. Antibiot. 60(1), 1-12 (2007).

410. Ohshiro, Taichi; Rudel, Lawrence L.; Ōmura, Satoshi; Tomoda, Hiroshi. Selectivity of microbial acyl-CoA: cholesterol acyltransferase inhibitors toward isozymes. J. Antibiot. 60(1), 43-51 (2007).

411. Ui, Hideaki; Ishiyama, Aki; Sekiguchi, Hitomi; Namatame, Miyuki; Nishihara, Aki; Takahashi, Yoko; Shiomi, Kazuro; Otoguro, Kazuhiko; Ōmura, Satoshi. Selective and potent in vitro antimalarial activities found in four microbial metabolites. J. Antibiot. 60(3), 220-222 (2007).

412. Iwatsuki, Masato; Uchida, Ryuji; Takakusagi, Yoichi; Matsumoto, Atsuko; Jiang, Cheng-Lin; Takahashi, Yoko; Arai, Masayoshi; Kobayashi, Susumu; Matsumoto, Makoto; Inokoshi, Junji; Tomoda, Hiroshi; Ōmura, Satoshi. Lariatins, novel anti-mycobacterial peptides with a lasso structure, produced by Rhodococcus jostii K01-B0171. J. Antibiot. 60(6), 357-363 (2007).

413. Fukumoto, Atsushi; Kim, Yong-Pil; Matsumoto, Atsuko; Takahashi, Yoko; Shiomi, Kazuro; Tomoda, Hiroshi; Ōmura, Satoshi. Cyslabdan, a new potentiator of imipenem activity against methicillin-resistant Staphylococcus aureus, produced by Streptomyces sp. K04-0144. I. Taxonomy, fermentation, isolation and structural elucidation. J. Antibiot. 61(1), 1-6 (2008).

414. Fukumoto, Atsushi; Kim, Yong-Pil; Hanaki, Hideaki; Shiomi, Kazuro; Tomoda, Hiroshi; Ōmura, Satoshi. Cyslabdan, a new potentiator of imipenem activity against Methicillin-resistant Staphylococcus aureus, produced by Streptomyces sp. K04-0144. II. Biological activities. J. Antibiot. 61(1), 7-10 (2008).

415. Sunazuka, Toshiaki; Shudo, Hiroko; Nagai, Kenichiro; Yoshida, Kiminari; Yamaguchi, Yukie; Hanaki, Hideaki; Ōmura, Satoshi. 4 "'- $N$-demethylspiramycin derivatives: synthesis and evaluation of effectiveness against drug-resistant bacteria. J. Antibiot. 61(3), 175-184 (2008).

416. Iwatsuki, Masato; Uchida, Ryuji; Yoshijima, Hitomi; Ui, Hideaki; Shiomi, Kazuro; Matsumoto, Atsuko; Takahashi, Yoko; Abe, 
Akio; Tomoda, Hiroshi; Ōmura, Satoshi. Guadinomines, type III secretion system inhibitors, produced by Streptomyces sp. K01-0509. I. Taxonomy, fermentation, isolation and biological properties. J. Antibiot. 61(4), 222-229 (2008).

417. Iwatsuki, Masato; Uchida, Ryuji; Yoshijima, Hitomi; Ui, Hideaki; Shiomi, Kazuro; Kim, Yong-Pil; Hirose, Tomoyasu; Sunazuka, Toshiaki; Abe, Akio; Tomoda, Hiroshi; Ōmura, Satoshi. Guadinomines, type III secretion system inhibitors, produced by Streptomyces sp. K01-0509. II. Physico-chemical properties and structure elucidation. J. Antibiot. 61(4), 230-236 (2008).

418. Ishiyama, Aki; Otoguro, Kazuhiko; Namatame, Miyuki; Nishihara, Aki; Furusawa, Toshiaki; Takahashi, Yoko; Masuma, Rokuro; Shiomi, Kazuro; Ōmura, Satoshi. Simaomicin $\alpha$ : effects on the cell cycle of synchronized, cultured Plasmodium falciparum. J. Antibiot. 61(4), 254-257 (2008).

419. Kojima, Yasuhiro; Sunazuka, Toshiaki; Nagai, Kenichiro; Julfakyan, Khachatur; Fukuda, Takashi; Tomoda, Hiroshi; Ōmura, Satoshi: total synthesis of malformin C, an inhibitor of bleomycin-induced G2 arrest. J. Antibiot. 61(5), 297-302 (2008).

420. Matsuda, Daisuke; Namatame, Ichiji; Ohshiro, Taichi; Ishibashi, Shun; Ōmura, Satoshi; Tomoda, Hiroshi. Anti-atherosclerotic activity of triacsin C, an acyl-CoA synthetase inhibitor. J. Antibiot. 61(5), 318-321 (2008).

421. Otoguro, Kazuhiko; Ishiyama, Aki; Namatame, Miyuki; Nishihara, Aki; Furusawa, Toshiaki; Masuma, Rokuro; Shiomi, Kazuro; Takahashi, Yoko; Yamada, Haruki; Ōmura, Satoshi. Selective and potent in vitro antitrypanosomal activities of ten microbial metabolites. J. Antibiot. 61(6), 372-378 (2008).

422. Yamazaki, Hiroyuki; Koyama, Nobuhiro; Ōmura, Satoshi; Tomoda, Hiroshi. Structure-activity relationships of stemphones, potentiators of imipenem activity against methicillin-resistant Staphylococcus aureus. J. Antibiot. 61(7), 426-441 (2008).

423. Ohshiro, Taichi; Ohte, Satoshi; Matsuda, Daisuke; Ohtawa, Masaki; Nagamitsu, Tohru; Sunazuka, Toshiaki; Harigaya, Yoshihiro; Rudel, Lawrence L.; Ōmura, Satoshi; Tomoda, Hiroshi. Selectivity of pyripyropene derivatives in inhibition toward acyl-CoA: cholesterol acyltransferase 2 isozyme. J. Antibiot. 61(8), 503-508 (2008).

424. Fukuda, Takashi; Hasegawa, Yoko; Sakabe, Yasunari; Tomoda, Hiroshi; Ōmura, Satoshi. Citrinamides, new potentiators of antifungal miconazole activity, produced by Penicillium sp. FKI-1938. J. Antibiot. 61(9), 550-555 (2008).

425. Ishiyama, Aki; Otoguro, Kazuhiko; Namatame, Miyuki; Nishihara, Aki; Furusawa, Toshiaki; Masuma, Rokuro; Shiomi, Kazuro; Takahashi, Yoko; Ichimura, Michio; Yamada, Haruki; Ömura, Satoshi. In vitro and in vivo antitrypanosomal activity of two microbial metabolites, KS-505a and alazopeptin. J. Antibiot. 61(10), 627-632 (2008).

426. Nakano, Hirofumi; Ōmura, Satoshi. Chemical biology of natural indolocarbazole products: 30 years since the discovery of staurosporine. J. Antibiot. 62(1), 17-26 (2009).

427. Inokoshi, Junji; Kawamoto, Kyosuke; Takagi, Yoichi; Matsuhama, Maki; Ōmura, Satoshi; Tomoda, Hiroshi. Expression of two human acyl-CoA: diacylglycerol acyltransferase isozymes in yeast and selectivity of microbial inhibitors toward the isozymes. J. Antibiot. 62(1), 51-54 (2009).

428. Nagamitsu, Tohru; Marumoto, Kaori; Nagayasu, Asami; Fukuda, Takeo; Arima, Shiho; Uchida, Ryuji; Ohshiro, Taichi; Harigaya, Yoshihiro; Tomoda, Hiroshi; Ōmura, Satoshi. Total synthesis of amidepsine $\mathrm{B}$ and revision of its absolute configuration. J. Antibiot. 62(2), 69-74 (2009).
429. Yamazaki, Hiroyuki; Kobayashi, Kakeru; Matsuda, Daisuke; Nonaka, Kenichi; Masuma, Rokuro; Ōmura, Satoshi; Tomoda, Hiroshi. Pentacecilides, new inhibitors of lipid droplet formation in mouse macrophages, produced by Penicillium cecidicola FKI-3765-1: I. Taxonomy, fermentation, isolation and biological properties. J. Antibiot. 62(4), 195-200 (2009).

430. Yamazaki, Hiroyuki; Ōmura, Satoshi; Tomoda, Hiroshi. Pentacecilides, new inhibitors of lipid droplet formation in mouse macrophages produced by Penicillium cecidicola FKI-3765-1: II. Structure elucidation. J. Antibiot. 62(4), 207-211 (2009).

431. Hirose, Tomoyasu; Sunazuka, Toshiaki; Sugawara, Akihiro; Endo, Ayako; Iguchi, Kanami; Yamamoto, Tsuyoshi; Ui, Hideaki; Shiomi, Kazuro; Watanabe, Takeshi; Sharpless, K. Barry; Ōmura, Satoshi. Chitinase inhibitors: extraction of the active framework from natural argifin and use of in situ click chemistry. J. Antibiot. 62(5), 277-282 (2009).

432. Ohtawa, Masaki; Ogihara, Satoru; Sugiyama, Kouhei; Shiomi, Kazuro; Harigaya, Yoshihiro; Nagamitsu, Tohru; Ōmura, Satoshi. Enantioselective total synthesis of atpenin A5. J. Antibiot. 62(6), 289-294 (2009).

433. Ishiyama, Aki; Otoguro, Kazuhiko; Iwatsuki, Masahito; Namatame, Miyuki; Nishihara, Aki; Nonaka, Kenichi; Kinoshita, Yuta; Takahashi, Yoko; Masuma, Rokuro; Shiomi, Kazuro; Yamada, Haruki; Ōmura, Satoshi. In vitro and in vivo antitrypanosomal activities of three peptide antibiotics: leucinostatin A and B, alamethicin I and tsushimycin. J. Antibiot. 62(6), 303-308 (2009).

434. Yamazaki, Hiroyuki; Nonaka, Kenichi; Masuma, Rokuro; Ōmura, Satoshi; Tomoda, Hiroshi. Xanthoradones, new potentiators of imipenem activity against methicillin-resistant Staphylococcus aureus, produced by Penicillium radicum FKI-3765-2: I. Taxonomy, fermentation, isolation and biological properties. J. Antibiot. 62(8), 431-434 (2009).

435. Yamazaki, Hiroyuki; Ōmura, Satoshi; Tomoda, Hiroshi. Xanthoradones, new potentiators of imipenem activity against methicillin-resistant Staphylococcus aureus, produced by Penicillium radicum FKI-3765-2: II. Structure elucidation. J. Antibiot. 62(8), 435-437 (2009).

436. Hirose, Tomoyasu; Sunazuka, Toshiaki; Sugawara, Akihiro; Noguchi, Yoshihiko; Tanaka, Toshiaki; Iguchi, Kanami; Yamamoto, Tsuyoshi; Gouda, Hiroaki; Shiomi, Kazuro; Ōmura, Satoshi. Solid-phase total synthesis of the chitinase inhibitor Argadin using a supported acetal resin. J. Antibiot. 62(9), 495-500 (2009).

437. Kojima, Yasuhiro; Sunazuka, Toshiaki; Nagai, Kenichiro; Hirose, Tomoyasu; Namatame, Miyuki; Ishiyama, Aki; Otoguro, Kazuhiko; Ōmura, Satoshi. Solid-phase synthesis and biological activity of malformin C and its derivatives. J. Antibiot. 62(12), 681-686 (2009).

438. Inokoshi, Junji; Takagi, Yoichi; Uchida, Ryuji; Masuma, Rokuro; Ōmura, Satoshi; Tomoda, Hiroshi. Production of a new type of amidepsine with a sugar moiety by static fermentation of Humicola sp. FO-2942. J. Antibiot. 63(1), 9-16 (2010).

439. Shiomi, Kazuro; Matsui, Ryosuke; Kakei, Atsuo; Yamaguchi, Yuichi; Masuma, Rokuro; Hatano, Hiroko; Arai, Noriko; Isozaki, Miki; Tanaka, Haruo; Kobayashi, Susumu; Turberg, Andreas; Ōmura, Satoshi. Verticilide, a new ryanodine-binding inhibitor, produced by Verticillium sp. FKI-1033. J. Antibiot. 63(2), 77-82 (2010). 
440. Uchida, Ryuji; Iwatsuki, Masato; Kim, Yong-Pil; Ohte, Satoshi; Ōmura, Satoshi; Tomoda, Hiroshi. Nosokomycins, new antibiotics discovered in an in vivo-mimic infection model using silkworm larvae. I: Fermentation, isolation and biological properties. J. Antibiot. 63(4), 151-155 (2010).

441. Uchida, Ryuji; Iwatsuki, Masato; Kim, Yong-Pil; Ōmura, Satoshi; Tomoda, Hiroshi. Nosokomycins, new antibiotics discovered in an in vivo-mimic infection model using silkworm larvae. II. Structure elucidation. J. Antibiot. 63(4), 157-163 (2010).

442. Koyama, Nobuhiro; Kojima, Shigenobu; Nonaka, Kenichi; Masuma, Rokuro; Matsumoto, Makoto; Ōmura, Satoshi; Tomoda, Hiroshi. Calpinactam, a new anti-mycobacterial agent, produced by Mortierella alpina FKI-4905. J. Antibiot. 63(4), 183-186 (2010).

443. Otoguro, Kazuhiko; Iwatsuki, Masato; Ishiyama, Aki; Namatame, Miyuki; Nishihara-Tsukashima, Aki; Sato, Seigo; Hatsu, Masahiro; Iinuma, Hironobu; Shibahara, Seiji; Nimura, Satoshi; Kondo, Shinichi; Yamada, Haruki; Ōmura, Satoshi. In vitro and in vivo antiprotozoal activities of bispolides and their derivatives. J. Antibiot. 63(5), 275-277 (2010).

444. Yamazaki, Hiroyuki; Ōmura, Satoshi; Tomoda, Hiroshi. Xanthoradone $\mathrm{C}$, a new potentiator of imipenem activity against methicillin-resistant Staphylococcusaureus, produced by Penicillium radicum FKI-3765-2. J. Antibiot. 63(6), 329-330 (2010).

445. Iwatsuki, Masato; Kinoshita, Yuta; Niitsuma, Megumi; Hashida, Junko; Mori, Mihoko; Ishiyama, Aki; Namatame, Miyuki; Nishihara-Tsukashima, Aki; Nonaka, Kenichi; Masuma, Rokuro; Otoguro, Kazuhiko; Yamada, Haruki; Shiomi, Kazuro; Ōmura, Satoshi. Antitrypanosomal peptaibiotics, trichosporins B-VIIa and B-VIIb, produced by Trichoderma polysporum FKI-4452. J. Antibiot. 63(6), 331-333 (2010).

446. Otoguro, Kazuhiko; Iwatsuki, Masato; Ishiyama, Aki; Namatame, Miyuki; Nishihara-Tukashima, Aki; Shibahara, Seiji; Kondo, Shinichi; Yamada, Haruki; Ōmura, Satoshi. Promising lead compounds for novel antiprotozoals. J. Antibiot. 63(7), 381-384 (2010)

447. Ikeda, Shinsuke; Hanaki, Hideaki; Yanagisawa, Chie; Ikeda-Dantsuji, Yurika; Matsui, Hidehito; Iwatsuki, Masato; Shiomi, Kazuro; Nakae, Taiji; Sunakawa, Keisuke; Ōmura, Satoshi. Identification of the active component that induces vancomycin resistance in MRSA. J. Antibiot. 63(9), 533-538 (2010).

448. Hashida, Junko; Niitsuma, Megumi; Iwatsuki, Masato; Mori, Mihoko; Ishiyama, Aki; Namatame, Miyuki; Nishihara-Tsukashima, Aki; Nonaka, Kenichi; Ui, Hideaki; Masuma, Rokuro; Otoguro, Kazuhiko; Yamada, Haruki; Shiomi, Kazuro; Ōmura, Satoshi. Pyrenocine I, a new pyrenocine analog produced by Paecilomyces sp. FKI-3573. J. Antibiot. 63 (9), 559-561 (2010).

449. Otoguro, Kazuhiko; Ishiyama, Aki; Iwatsuki, Masato; Namatame, Miyuki; Nishihara-Tukashima, Aki; Nakashima, Takuji; Shibahara, Seiji; Kondo, Shinichi; Yamada, Haruki; Ōmura, Satoshi. In vitro and in vivo anti-Trypanosoma brucei activities of phenazinomycin and related compounds. J. Antibiot. 63(9), 579-581 (2010).

450. Iwatsuki, Masato; Otoguro, Kazuhiko; Ishiyama, Aki; Namatame, Miyuki; Nishihara-Tukashima, Aki; Hashida, Junko; Nakashima, Takuji; Masuma, Rokuro; Takahashi, Yoko; Yamada, Haruki; Ōmura, Satoshi. In vitro antitrypanosomal activity of 12 low-molecular-weight antibiotics and observations of structure/activity relationships. J. Antibiot. 63(10), 619-622 (2010).

451. Takahashi, Atsushi; Inokoshi, Junji; Tsunoda, Masaru; Suzuki, Kaoru; Takenaka, Akio; Sekiguchi, Takeshi; Ōmura, Satoshi; Tanaka, Haruo. Actinohivin: specific amino acid residues essential for anti-HIV activity. J. Antibiot. 63(11), 661-665 (2010).

452. Niitsuma, Megumi; Hashida, Junko; Iwatsuki, Masato; Mori, Mihoko; Ishiyama, Aki; Namatame, Miyuki; Nishihara-Tsukashima, Aki; Matsumoto, Atsuko; Takahashi, Yoko; Yamada, Haruki; Otoguro, Kazuhiko; Shiomi, Kazuro; Ōmura, Satoshi. Sinefungin VA and dehydrosinefungin V, new antitrypanosomal antibiotics produced by Streptomyces sp. K05-0178. J. Antibiot. 63(11), 673-679 (2010).

453. Iwatsuki, Masato; Takada, Shohei; Mori, Mihoko; Ishiyama, Aki; Namatame, Miyuki; Nishihara-Tsukashima, Aki; Nonaka, Kenichi; Masuma, Rokuro; Otoguro, Kazuhiko; Shiomi, Kazuro; Ömura, Satoshi. In vitro and in vivo antimalarial activity of puberulic acid and its new analogs, viticolins A-C, produced by Penicillium sp. FKI-4410. J. Antibiot. 64(2), 183-188 (2011).

454. Kimura, Kyota; Iwatsuki, Masato; Nagai, Takeshi; Matsumoto, Atsuko; Takahashi, Yoko; Shiomi, Kazuro; Ōmura, Satoshi; Abe, Akio. A small-molecule inhibitor of the bacterial type III secretion system protects against in vivo infection with Citrobacter rodentium. J. Antibiot. 64(2), 197-203 (2011).

455. Inahashi, Yuki; Matsumoto, Atsuko; Ōmura, Satoshi; Takahashi, Yoko. Streptosporangium oxazolinicum sp. nov., a novel endophytic actinomycete producing new antitrypanosomal antibiotics, spoxazomicins. J. Antibiot. 64(4), 297-302 (2011).

456. Inahashi, Yuki; Iwatsuki, Masato; Ishiyama, Aki; Namatame, Miyuki; Nishihara-Tsukashima, Aki; Matsumoto, Atsuko; Hirose, Tomoyasu; Sunazuka, Toshiaki; Yamada, Haruki; Otoguro, Kazuhiko; Takahashi, Yoko; Ōmura, Satoshi; Shiomi, Kazuro. Spoxazomicins A-C, novel antitrypanosomal alkaloids produced by an endophytic actinomycete, Streptosporangium oxazolinicum K07-0460 ${ }^{\mathrm{T}}$. J. Antibiot. 64(4), 303-307 (2011).

457. Ishiyama, Aki; Iwatsuki, Masato; Namatame, Miyuki; Nishihara-Tsukashima, Aki; Sunazuka, Toshiaki; Takahashi, Yoko; Ōmura, Satoshi; Otoguro, Kazuhiko. Borrelidin, a potent antimalarial: stage-specific inhibition profile of synchronized cultures of Plasmodium falciparum. J. Antibiot. 64(5), 381-384 (2011).

458. Ohte, Satoshi; Matsuda, Daisuke; Uchida, Ryuji; Nonaka, Kenichi; Masuma, Rokuro; Ōmura, Satoshi; Tomoda, Hiroshi. Dinapinones, novel inhibitors of triacylglycerol synthesis in mammalian cells, produced by Penicillium pinophilum FKI-3864. J. Antibiot. 64(7), 489-494 (2011).

459. Takahashi, Atsushi; Inokoshi, Junji; Hachiya, Atsuko; Oka, Shinichi; Ōmura, Satoshi; Tanaka, Haruo. The high mannosetype glycan binding lectin actinohivin: dimerization greatly improves anti-HIV activity. J. Antibiot. 64(8), 551-557 (2011).

460. Nonaka, Kenichi; Abe, Tadahide; Iwatsuki, Masato; Mori, Mihoko; Yamamoto, Tsuyoshi; Shiomi, Kazuro; Ōmura, Satoshi; Masuma, Rokuro. Enhancement of metabolites productivity of Penicillium pinophilum FKI-5653, by co-culture with Trichoderma harzianum FKI-5655. J. Antibiot. 64(12), 769-774 (2011).

461. Ugaki, Narihiro; Matsuda, Daisuke; Yamazaki, Hiroyuki; Nonaka, Kenichi; Masuma, Rokuro; Ōmura, Satoshi; Tomoda, Hiroshi. New isochaetochromin, an inhibitor of triacylglycerol synthesis in mammalian cells, produced by Penicillium sp. 
FKI-4942: I. Taxonomy, fermentation, isolation and biological properties. J. Antibiot. 65(1), 15-19 (2012).

462. Ishiyama, Aki; Otoguro, Kazuhiko; Iwatsuki, Masato; Namatame, Miyuki; Nishihara-Tsukashima, Aki; Takahashi, Yoko; Onodera, Hideyuki; Yamada, Haruki; Ōmura, Satoshi. In vitro antitrypanosomal activity of five low-MW antibiotics. J. Antibiot. 65(2), 113-114 (2012).

463. Ishii, Takahiro; Nonaka, Kenichi; Iwatsuki, Masato; Masuma, Rokuro; Ōmura, Satoshi; Shiomi, Kazuro. Virgaricin produced by Virgaria sp. FKI-4860. J. Antibiot. 65(3), 139-141 (2012).

464. Iwatsuki, Masato; Nishihara-Tsukashima, Aki; Ishiyama, Aki; Namatame, Miyuki; Watanabe, Yoshio; Handasah, Siti; Pranamuda, Hardaning; Marwoto, Bambang; Matsumoto, Atsuko; Takahashi, Yoko; Otoguro, Kazuhiko; Ōmura, Satoshi. Jogyamycin, a new antiprotozoal aminocyclopentitol antibiotic, produced by Streptomyces sp. a-WM-JG-16.2. J. Antibiot. 65(3), 169-171 (2012).

465. Hashida, Junko; Niitsuma, Megumi; Iwatsuki, Masato; Mori, Mihoko; Ishiyama, Aki; Namatame, Miyuki; Nishihara-Tsukashima, Aki; Matsumoto, Atsuko; Ara, Ismet; Takahashi, Yoko; Yamada, Haruki; Otoguro, Kazuhiko; Shiomi, Kazuro; Ōmura, Satoshi. Panowamycins A and B, new antitrypanosomal isochromans produced by Streptomyces sp. K070010. J. Antibiot. 65(4), 197-202 (2012).

466. Koyama, Ryo; Matsumoto, Atsuko; Inahashi, Yuki; Ōmura, Satoshi; Takahashi, Yoko. Isolation of actinomycetes from the root of the plant, Ophiopogon japonicus, and proposal of two new species, Actinoallomurus liliacearum sp. nov. and Actinoallomurus vinaceus sp. nov. J. Antibiot. 65(7), 335-340 (2012).

467. Sugawara, Akihiro; Sueki, Akito; Hirose, Tomoyasu; Shima, Hideaki; Akagawa, Kiyoko S.; Ōmura, Satoshi; Sunazuka, Toshiaki. Novel 12-membered non-antibiotic macrolides, EM900 series with anti-inflammatory and/or immunomodulatory activity; synthesis, structure-activity relationships and in vivo study. J. Antibiot. 65(9), 487-490 (2012).

468. Suga, Takuya; Ishii, Takahiro; Iwatsuki, Masato; Yamamoto, Tsuyoshi; Nonaka, Kenichi; Masuma, Rokuro; Matsui, Hidehito; Hanaki, Hideaki; Ōmura, Satoshi; Shiomi, Kazuro. Aranorosin circumvents arbekacin-resistance in MRSA by inhibiting the bifunctional enzyme AAC (6')/APH (2"). J. Antibiot. 65(10), 527-529 (2012).

469. Tsutsui, Ayumi; Hirose, Tomoyasu; Ishiyama, Aki; Iwatsuki, Masato; Yokota, Arisa; Maruyama, Hitomi; Matsui, Hidehito; Otoguro, Kazuhiko; Hanaki, Hideaki; Ōmura, Satoshi; Sunazuka, Toshiaki. Antimalarial C-9 oxime derivatives from desmycosin, produced by click chemistry. J. Antibiot. 66(3), 191-194 (2013).

470. Nakashima, Takuji; Okuyama, Ryuki; Kamiya, Yoshiyuki; Matsumoto, Atsuko; Iwatsuki, Masato; Inahashi, Yuki; Yamaji, Kenzaburo; Takahashi, Yoko; Ōmura, Satoshi. Trehangelins A, B and $\mathrm{C}$, novel photo-oxidative hemolysis inhibitors produced by an endophytic actinomycete, Polymorphospora rubra K07-0510. J. Antibiot. 66(6), 311-317 (2013).

471. Umeyama, Akemi; Takahashi, Koichi; Grudniewska, Aleksandra; Shimizu, Mina; Hayashi, Sayaka; Kato, Masayuki; Okamoto, Yasuko; Suenaga, Midori; Ban, Sayaka; Kumada, Toshio; Ishiyama, Aki; Iwatsuki, Masato; Otoguro, Kazuhiko; Ōmura, Satoshi; Hashimoto, Toshihiro. In vitro antitrypanosomal activity of the cyclodepsipeptides, cardinalisamides A-C, from the insect pathogenic fungus Cordyceps cardinalis NBRC 103832. J. Antibiot. 67(2), 163-166 (2014).
472. Sugamata, Ryuichi; Sugawara, Akihiro; Nagao, Tomokazu; Suzuki, Koya; Hirose, Tomoyasu; Yamamoto, Ki-ichi; Oshima, Masamichi; Kobayashi, Kazuo; Sunazuka, Toshiaki; Akagawa, Kiyoko S.; Ōmura, Satoshi; Nakayama, Toshinori; Suzuki, Kazuo. Leucomycin $\mathrm{A}_{3}$, a 16-membered macrolide antibiotic, inhibits influenza A virus infection and disease progression. J. Antibiot. 67 (3), 213-222 (2014).

473. Nakashima, Takuji; Iwatsuki, Masato; Ochiai, Junya; Kamiya, Yoshiyuki; Nagai, Kenichiro; Matsumoto, Atsuko; Ishiyama, Aki; Otoguro, Kazuhiko; Shiomi, Kazuro; Takahashi, Yoko; Ōmura, Satoshi. Mangromicins A and B. structure and antitrypanosomal activity of two new cyclopentadecane compounds from Lechevalieria aerocolonigenes K10-0216. J. Antibiot. 67(3), 253-260 (2014).

474. Nakashima, Takuji; Kamiya, Yoshiyuki; Iwatsuki, Masato; Takahashi, Yoko; Ōmura, Satoshi. Mangromicins, six new antioxidative agents isolated from a culture broth of the actinomycete, Lechevalieria aerocolonigenes K10-0216. J. Antibiot. 67(7), 533-539 (2014).

475. Ishiyama, Aki; Iwatsuki, Masato; Yamamoto, Tsuyoshi; Miura, Hiromi; Ōmura, Satoshi; Otoguro, Kazuhiko. Antimalarial tropones and their Plasmodium falciparum glyoxalase I ( $p f \mathrm{GLOI})$ inhibitory activity. J. Antibiot. 67(7), 545-547 (2014).

476. Nonaka, Kenichi; Todaka, Nemuri; Ōmura, Satoshi; Masuma, Rokuro. Combination cellulose plate (non-agar solid support) and agar plate method improves isolation of fungi from soil. J. Antibiot. 67(11), 755-761 (2014).

477. Nakashima, Takuji; Kamiya, Yoshiyuki; Iwatsuki, Masato; Sato, Noriko; Takahashi, Yoko; Ōmura, Satoshi. Mangromicin C, a new analog of mangromicin. J. Antibiot. 68(3), 220-222 (2015).

478. Take, Akira; Matsumoto, Atsuko; Ōmura, Satoshi; Takahashi, Yoko. Streptomyces lactacystinicus sp. nov. and Streptomyces cyslabdanicus sp. nov., producing lactacystin and cyslabdan, respectively. J. Antibiot. 68(5), 322-327 (2015).

479. Nakashima, Takuji; Kamiya, Yoshiyuki; Yamaji, Kenzaburo; Iwatsuki, Masato; Sato, Noriko; Takahashi, Yoko; Ōmura, Satoshi. New steroidal compounds from an actinomycete strain, Lechevalieria aerocolonigenes K10-0216. J. Antibiot. 68(5), 348-350 (2015).

480. Kaifuchi, Satoru; Mori, Mihoko; Nonaka, Kenichi; Masuma, Rokuro; Ōmura, Satoshi; Shiomi, Kazuro. Sartorypyrone D: a new NADH-fumarate reductase inhibitor produced by Neosartorya fischeri FO-5897. J. Antibiot. 68(6), 403-405 (2015).

481. Ishiyama, Aki; Iwatsuki, Masato; Hokari, Rei; Sawa, Masaaki; Ōmura, Satoshi; Otoguro, Kazuhiko. Antimalarial activity of kinase inhibitor, nilotinib, in vitro and in vivo. J. Antibiot. 68(7), 469-472 (2015).

482. Nonaka, Kenichi; Chiba, Takuya; Suga, Takuya; Asami, Yukihiro; Iwatsuki, Masato; Masuma, Rokuro; Ōmura, Satoshi; Shiomi, Kazuro. Coculnol, a new penicillic acid produced by a coculture of Fusarium solani FKI-6853 and Talaromyces sp. FKA-65. J. Antibiot. 68(8), 530-532 (2015).

483. Nonaka, Kenichi; Iwatsuki, Masato; Horiuchi, Syunsuke; Shiomi, Kazuro; Ōmura, Satoshi; Masuma, Rokuro. Induced production of BE-31405 by co-culturing of Talaromyces siamensis FKA-61 with a variety of fungal strains. J. Antibiot. 68(9), 573-578 (2015).

484. Ishii, Takahiro; Nonaka, Kenichi; Sugawara, Akihiro; Iwatsuki, Masato; Masuma, Rokuro; Hirose, Tomoyasu; Sunazuka, Toshiaki; Ōmura, Satoshi; Shiomi, Kazuro. Cinatrins D and E, 
and virgaricin B, three novel compounds produced by a fungus, Virgaria boninensis FKI-4958. J. Antibiot. 68(10), 633-637 (2015).

485. Suga, Takuya; Asami, Yukihiro; Hashimoto, Shohei; Nonaka, Kenichi; Iwatsuki, Masato; Nakashima, Takuji; Sugahara, Ryohei; Shiotsuki, Takahiro; Yamamoto, Takenori; Shinohara, Yasuo; Ichimaru, Naoya; Murai, Masatoshi; Miyoshi, Hideto; Ōmura, Satoshi; Shiomi, Kazuro. Ascosteroside C, a new mitochondrial respiration inhibitor discovered by pesticidal screening using recombinant Saccharomyces cerevisiae. J. Antibiot. 68(10), 649-652 (2015).

486. Seki, Tamae; Matsumoto, Atsuko; Ōmura, Satoshi; Takahashi, Yoko. Distribution and isolation of strains belonging to the order Solirubrobacterales. J. Antibiot. 68(12), 763-766 (2015).

487. Sugawara, Akihiro; Shima, Hideaki; Sueki, Akito; Hirose, Tomoyasu; Matsui, Hidehito; Nakano, Hayato; Hanaki, Hideaki; Akagawa, Kiyoko S.; Ōmura, Satoshi; Sunazuka, Toshiaki. Non-antibiotic 12-membered macrolides: design, synthesis and biological evaluation in a cigarette-smoking model. J. Antibiot. 69 (4), 319-326 (2016).

488. Suga, Takuya; Shiina, Mayu; Asami, Yukihiro; Iwatsuki, Masato; Yamamoto, Tsuyoshi; Nonaka, Kenichi; Masuma, Rokuro; Matsui, Hidehito; Hanaki, Hideaki; Iwamoto, Susumu; Onodera, Hideyuki; Kazuro, hiomi; Ōmura, Satoshi. Paraphaeosphaeride $\mathrm{D}$ and berkleasmin $\mathrm{F}$, new circumventors of arbekacin resistance in MRSA, produced by Paraphaeosphaeria sp. TR-022. J. Antibiot. 69(8), 605-610 (2016).

489. Nakashima, Takuji; Miyano, Rei; Iwatsuki, Masato; Shirahata, Tatsuya; Kimura, Toru; Asami, Yukihiro; Kobayashi, Yoshinori; Shiomi, Kazuro; Petersson, George A.; Takahashi, Yoko; Ōmura, Satoshi. Iminimycin A, the new iminium metabolite produced by Streptomyces griseus OS-3601. J. Antibiot. 69(8), 611-615 (2016).

490. Shiina, Mayu; Suga, Takuya; Asami, Yukihiro; Nonaka, Kenichi; Iwatsuki, Masato; Ōmura, Satoshi; Shiomi, Kazuro. Euvesperins A and B, new circumventors of arbekacin resistance in MRSA, produced by Metarhizium sp. FKI-7236. J. Antibiot. 69(9), 719-722 (2016).

491. Kimura, Toru; Iwatsuki, Masato; Asami, Yukihiro; Ishiyama, Aki; Hokari, Rei; Otoguro, Kazuhiko; Matsumoto, Atsuko; Sato, Noriko; Shiomi, Kazuro; Takahashi, Yoko; Ōmura, Satoshi; Nakashima, Takuji. Anti-trypanosomal compound, sagamilactam, a new polyene macrocyclic lactam from Actinomadura sp. K13-0306. J. Antibiot. 69(11), 818-824 (2016).

492. Horikoshi, Ryo; Goto, Kimihiko; Mitomi, Masaaki; Oyama, Kazuhiko; Sunazuka, Toshiaki; Ōmura, Satoshi. Identification of pyripyropene $\mathrm{A}$ as a promising insecticidal compound in a microbial metabolite screening. J. Antibiot. 70(3), 272-276 (2017).

493. Watanabe, Yoshihiro; Suga, Takuya; Narusawa, Satomi; Iwatsuki, Masato; Nonaka, Kenichi; Nakashima, Takuji; Shinohara, Yasuo; Shiotsuki, Takahiro; Ichimaru, Naoya; Miyoshi, Hideto; Asami, Yukihiro; Ōmura, Satoshi; Shiomi, Kazuro. Decatamariic acid, a new mitochondrial respiration inhibitor discovered by pesticidal screening using drug-sensitive Saccharomyces cerevisiae. J. Antibiot. 70(4), 395-399 (2017).

494. Fukumoto, Atsushi; Kim, Yong-Pil; Matsumoto, Atsuko; Takahashi, Yoko; Suzuki, Makoto; Onodera, Hideyuki; Tomoda, Hiroshi; Matsui, Hidehito; Hanaki, Hideaki; Iwatsuki, Masato; Ōmura, Satoshi; Shiomi, Kazuro. Naphthacemycins, novel circumventors of $\beta$-lactam resistance in MRSA, produced by Streptomyces sp. KB-3346-5. I. The taxonomy of the producing strain, and the fermentation, isolation and antibacterial activities. J. Antibiot. (in press).

495. Fukumoto, Atsushi; Kim, Yong-Pil; Iwatsuki, Masato; Hirose, Tomoyasu; Sunazuka, Toshiaki; Hanaki, Hideaki; Ōmura, Satoshi; Shiomi, Kazuro. Naphthacemycins, novel circumventors of $\beta$-lactam resistance in MRSA, produced by Streptomyces sp. KB-3346-5. II. Structure elucidation. J. Antibiot. (in press).

496. Hirose, Tomoyasu; Kojima, Yasuhiro; Matsui, Hidehito; Hanaki, Hideaki; Iwatsuki, Masato; Shiomi, Kazuro; Ōmura, Satoshi; Sunazuka, Toshiaki. Total synthesis of $( \pm)$-naphthacemycin $A_{9}$, possessing both antibacterial activity against methicillin-resistant Staphylococcus aureus (MRSA) and circumventing effect of $\beta$-lactam resistance. J. Antibiot. (in press).

497. Ohtawa, Masaki; Arima, Shiho; Shimizu, Risa; Hanatani, Naomi; Shimizu, Eri; Shiomi, Kazuro; Kita, Kiyoshi; Ōmura, Satoshi; Nagamitsu, Tohru. Development of a new air-stable structure-simplified nafuredin- $\gamma$ analog as a potent and selective nematode complex I inhibitor. J. Antibiot. (in press).

498. Katz, Naftale; Araújo, Neusa; Coelho, Paulo Marcos Zech; Morel, Carlos Medicis; Linde-Arias, Ana Rosa; Yamada, Takeshi; Horimatsu, Yuki; Suzuki, Koh; Sunazuka, Toshiaki; Ōmura Satoshi. Ivermectin efficacy against Biomphalaria, intermediate host snail vectors of Schistosomiasis. J. Antibiot. (in press).

499. Shirahata, Tatsuya; Ino, Chieko; Mizuno, Fumi; Asada, Yoshihisa; Hirotani, Masao; Petersson, George A.; Ōmura, Satoshi; Yoshikawa, Takafumi; Kobayashi, Yoshinori. $\gamma$-Ionylidene-type sesquiterpenoids possessing antimicrobial activity against Porphyromonas gingivalis, from Phellinus linteus and their absolute structure determination. J. Antibiot. (in press). 